\title{
STABILIZATION TO TRAJECTORIES FOR PARABOLIC EQUATIONS
}

\author{
DUY PHAN AND SÉRGIO S. RODRIGUES
}

\begin{abstract}
The feedback exponential stabilization to trajectories for semilinear parabolic equations in a given bounded domain is addressed. The controls take values in a finite-dimensional space and are supported in a small region. Both internal and boundary controls are considered.
\end{abstract}

MSC2010: 93D15, 93B52, 35K58

Keywords: feedback stabilization, internal and boundary controls, parabolic equations

\section{Contents}

1. Introduction.

2. Reduction to stabilization to zero

2.1. The case of internal controls

2.2. The case of boundary controls

2.3. Stabilization to zero

3. Internal stabilization of the linearized system

3.1. Weak solutions

3.2. Null controllability

3.3. Stabilization to zero by finite dimensional controls

3.4. The dimension of the control

3.5. Feedback stabilizing rule and Riccati equation

3.6. Dependence of the transient bound on the exponential rate

3.7. Remark on the viscosity coefficient

4. Local internal stabilization of the nonlinear system

4.1. Strong solutions for the linearized systems

4.2. Fixed point argument

5. Local internal stabilization to trajectories

6. Example. Polynomial nonlinearities

7. Boundary stabilization

7.1. Weak solutions

7.2. Strong solutions

7.3. Null controllability

7.4. Stabilization to zero by finite dimensional controls

7.5. Feedback stabilizing rule and Riccati equation

7.6. The nonlinear systems

7.7. Back to original time. Stabilization to trajectories

8. Discretization of the linear systems

8.1. Discretization in space

8.2. Discretization in time

9. Numerical examples

9.1. Testing with a family of functions $(\hat{a}, \hat{b})$.

9.2. Increasing the number of actuators

9.3. Comparing with another placement of the actuators

9.4. Dependence of the transient bound on the desired decreasing rate

9.5. On the parameter $\varsigma$

9.6. On the feedback nature of the control

9.7. Switching the control off/on

9.8. A nonlinear example

9.9. The discretization error

References

The authors acknowledge support from the Austrian Science Fund (FWF): P 26034-N25. 


\section{INTRODUCTION.}

We consider controlled parabolic equations, for time $t \geq 0$, in a smooth domain $\Omega \in \mathbb{R}^{d}$ located locally on one side of its boundary $\Gamma=\partial \Omega$, with $d$ a positive integer, either of the form

$$
\partial_{t} y-\nu \Delta y+f(y, \nabla y)+\sum_{i=1}^{M} u_{i} \Phi_{i}=0 ;\left.\quad y\right|_{\Gamma}=g
$$

or of the form

$$
\partial_{t} y-\nu \Delta y+f(y, \nabla y)=0 ;\left.\quad y\right|_{\Gamma}=g+\sum_{i=1}^{M} u_{i} \Psi_{i} .
$$

In the variables $(t, x, \bar{x}) \in(0,+\infty) \times \Omega \times \Gamma$, the unknown in the equation is the function $y=y(t, x) \in \mathbb{R}$. The diffusion coefficient $\nu>0$ is a positive constant; the functions $g=g(t, \bar{x}) \in \mathbb{R}$ and $f: \mathbb{R} \times \mathbb{R}^{d} \rightarrow \mathbb{R}$ are fixed.

In system (1) the functions $\Phi_{i}=\Phi_{i}(x)$ are given and will play the role of actuators, while in system (2) that role will be played by the given functions $\Psi_{i}=\Psi_{i}(\bar{x})$. Finally, $M$ is a positive integer and, in either system, $u=u(t) \in \mathbb{R}^{M}$ is a (control) vector function at our disposal.

The problem we address here is the local exponential stabilization to trajectories for systems (1) and (2). That is, given a positive constant $\lambda>0$ and a solution $\hat{y}(t)=\hat{y}(t, \cdot)$ of the (uncontrolled) system with $u=0$, we want to find a control function $u$ such that the solution $y(t):=y(t, \cdot)$ of the system, supplemented with the initial condition

$$
y(0):=y(0, x)=y_{0}(x),
$$

is defined on $[0,+\infty)$ and approaches $\hat{y}(t)$ exponentially with rate $\frac{\lambda}{2}$, provided $y(0)-\hat{y}(0)$ is small enough. In other words, for a suitable Banach space $X$ and positive constants $C$ and $\epsilon$, we want to have that

$$
|y(t)-\hat{y}(t)|_{X}^{2} \leq C \mathrm{e}^{-\lambda t}|y(0)-\hat{y}(0)|_{X}^{2}, \quad \text { provided } \quad|y(0)-\hat{y}(0)|_{X}<\epsilon
$$

with $\epsilon$ small enough. Notice that, the constants $C$ and $\epsilon$ may depend on $\lambda$, but neither on $\hat{y}(0)$ nor on $y(0)$.

We are particularly interested in actuators which are supported in a small domain: either supp $\Phi_{i} \subset \omega \subseteq \Omega$ or supp $\Psi_{i} \subset \mathcal{O} \subseteq \Gamma$, where $\omega$ and $\mathcal{O}$ are given open subsets of $\Omega$ and $\Gamma$, respectively.

By following the arguments in [BRS11, KR15b, KR15a, BKR15] we shall conclude that the answer is affirmative, for the case of system (1) under suitable conditions on the family of internal actuators $\mathcal{C}_{\omega}=\left\{\Phi_{i} \mid i \in\{1,2, \ldots, M\}\right\}$. Moreover the stabilizing control can be taken in feedback form $u(t)=K_{t}(y(t)-\hat{y}(t))$.

For the case of system (2) the answer is less straightforward from the available results in the Literature, however we shall show, by combining some of the arguments in Bad09, Rod14, Rod15a, that the answer is again affirmative provided suitable conditions are satisfied by the family of boundary actuators $\mathcal{C}_{\Gamma_{\mathrm{c}}}=\left\{\Psi_{i} \mid i \in\{1,2, \ldots, M\}\right\}$. In this case, the control can be taken in integral feedback form $\left.y\right|_{\Gamma}(t)=v_{0}+\int_{0}^{t} K_{\tau}(y(\tau)-\hat{y}(\tau)) \mathrm{d} \tau$, where $v_{0}$ may/must be taken in an appropriate space.

Considering finite-dimensional controls is important for applications because usually we have at our disposal only a finite number of actuators which we can tune. Suppose we can choose those (either internal or boundary) actuators from a family

$$
\mathcal{C}^{+\infty}=\left\{\Theta_{i} \mid i \in \mathbb{N}_{0}\right\}
$$


then we may ask how many of these actuators we need to stabilize the system. That is, what is $M$ such that $\mathcal{C}^{M}=\left\{\Theta_{i} \mid i \in\{1,2, \ldots, M\}\right\}$ allow us to stabilize the system. This question will be answered for suitable complete families. In this way we arrive to an estimate on the number of actuators we need to stabilize the system.

We will start by considering the linearization of systems (1) and (2) around $\hat{y}$ and construct a feedback rule stabilizing globally the solution $v$ of the linearized system to zero. That is,

$$
|v(t)(t)|_{X}^{2} \leq C \mathrm{e}^{-\lambda t}|v(0)|_{X}^{2}
$$

Another question we will address is how the constant $C=C(\lambda)$, in (4), does depend on $\lambda$. Some estimates will be given and the results of some numerical simulations will be presented for Riccati based feedback. This is motivated by a sufficient condition for exponential stabilization given in [BKR15] for the FitzHugh-Nagumo and RogersMcCulogh systems. Another motivation is that in general, the value $\epsilon$ in (3) will decrease as $C$ in (4) increases, that is the feedback control will work, for the nonlinear system, in a bigger neighborhood for a smaller $C$.

The rest of the paper is organized as follows. In Section 2 we reduce our problem to the stabilization to zero of the difference $z=y-\hat{y}$, and write the system for $z$ in an appropriate way. In Section 3 we deal with the internal stabilization to zero of the linearized system for the difference. In Section 4 we deal with the internal stabilization to zero of the nonlinear (full) system for the difference, under some conditions on the nonlinearity. In Section 6, as an example, we check the conditions in Section 4 for some nonlinearities of polynomial type. In Section 7 we deal with boundary controls. Finally, Sections 8 and 9 are concerned with the discretization of our equations and the presentation of the results of some numerical simulations.

Notation. We write $\mathbb{R}$ and $\mathbb{N}$ for the sets of real numbers and nonnegative integers, respectively, and we define $\mathbb{R}_{a}:=(a,+\infty)$ for all $a \in \mathbb{R}$, and $\mathbb{N}_{0}:=\mathbb{N} \backslash\{0\}$. We denote by $\Omega \subset \mathbb{R}^{n}, n \in \mathbb{N}_{0}$, a bounded domain with a smooth boundary $\Gamma=\partial \Omega$. Given a function $v:\left(t, x_{1}, x_{2}, \ldots, x_{n}\right) \mapsto v\left(t, x_{1}, x_{2}, \ldots, x_{n}\right) \in \mathbb{R}$, defined in an open subset of $\mathbb{R} \times \Omega$, its partial time derivative $\frac{\partial v}{\partial t}$ will be denoted by $\partial_{t} v$.

We use the standard notation for Bochner spaces $L^{p}(\Omega, X)$ where $\Omega \subseteq \mathbb{R}^{n}, n \in \mathbb{N}_{0}$, $p \in[1,+\infty]$, and $X$ is a Banach space. The spaces $L^{p}(\Omega)^{m}=L^{p}\left(\Omega, \mathbb{R}^{m}\right)$ will be denoted by simply $L^{p}$ whenever there is no ambiguity neither concerning the domain $\Omega$ nor the superscript $m \in \mathbb{N}_{0}$.

Given an open interval $I \subseteq \mathbb{R}$, and Banach spaces $X$ and $Y$, we write $W(I, X, Y):=$ $\left\{f \in L^{2}(I, X) \mid \partial_{t} f \in L^{2}(I, Y)\right\}$, where the derivative $\partial_{t} f$ is taken in the sense of distributions. This space is endowed with the natural norm $|f|_{W(I, X, Y)}:=\left(|f|_{L^{2}(I, X)}^{2}+\right.$ $\left.\left|\partial_{t} f\right|_{L^{2}(I, Y)}^{2}\right)^{1 / 2}$. The space of continuous linear mappings from $X$ into $Y$ will be denoted by $\mathcal{L}(X, Y)$. In case $X=Y$ we write $\mathcal{L}(X):=\mathcal{L}(X, X)$ instead. If the inclusion $X \subseteq Y$ is continuous, we write $X \hookrightarrow Y$; we write $X \stackrel{\mathrm{d}}{\hookrightarrow} Y$, respectively $X \stackrel{\mathrm{c}}{\hookrightarrow} Y$, if the inclusion is also dense, respectively compact. The kernel and range of a linear mapping $A: Z \rightarrow W$, between vector spaces $Z$ and $W$, will be denoted $\operatorname{Ker} A:=\{x \in Z \mid A x=0\}$ and $\operatorname{Ran} A:=\{A x \mid x \in Z\}$, respectively.

$\bar{C}_{\left[a_{1}, \ldots, a_{k}\right]}$ denotes a function of nonnegative variables $a_{j}$ that increases in each of its arguments, and $C, C_{i}, i=1,2, \ldots$, stand for positive constants. 


\section{Reduction to StABilization to ZERo}

Let $\hat{y}(t)$ solves the uncontrolled system. We we want the solution $y(t)$ to go to the reference trajectory $\hat{y}(t)$ exponentially, thus it is natural to consider the dynamics of the difference $y-\hat{y}$.

2.1. The case of internal controls. By direct computations, we find that $z:=y-\hat{y}$ solves

$$
\partial_{t} z-\nu \Delta z+f(y, \nabla y)-f(\hat{y}, \nabla \hat{y})+\sum_{i=1}^{M} u_{i} \Phi_{i}=0,\left.\quad z\right|_{\Gamma}=0 .
$$

Writing $\left(\xi^{1}, \xi^{2}\right) \in \mathbb{R} \times \mathbb{R}^{d}$ we denote $\partial_{1} f:=\frac{\partial f}{\partial_{\xi^{1}}}$ and $\partial_{2} f:=\frac{\partial f}{\partial_{\xi^{2}}}$. Formally, we can write

$$
\begin{aligned}
f(y, \nabla y)-f(\hat{y}, \nabla \hat{y}) & =:\left[\left.\left.\partial_{1} f\right|_{(\hat{y}, \nabla \hat{y})} \quad \partial_{2} f\right|_{(\hat{y}, \nabla \hat{y})}\left[\begin{array}{c}
z \\
\nabla z
\end{array}\right]+F_{\hat{y}}(z)\right. \\
& =\hat{a} z+\nabla \cdot(\hat{b} z)-\widehat{\mathcal{N}}(z) .
\end{aligned}
$$

with

$$
\hat{a}:=\left.\partial_{1} f\right|_{(\hat{y}, \nabla \hat{y})}-\left.\nabla \cdot \partial_{2} f\right|_{(\hat{y}, \nabla \hat{y})}, \quad \hat{b}:=\left.\partial_{2} f\right|_{(\hat{y}, \nabla \hat{y})}, \quad \text { and } \quad \hat{\mathcal{N}}(z)=-F_{\hat{y}}(z),
$$

where $\widehat{\mathcal{N}}(\cdot): \mathbb{R} \rightarrow \mathbb{R}$ is a nonlinear function if so is $y \mapsto f(y, \nabla y)$.

Rescaling time. By technical reasons, in order to use available results in the Literature (for the case $\nu=1$ ), it is convenient to rewrite the system as

$$
\partial_{\tau} \breve{z}-\Delta \breve{z}+\frac{1}{\nu} \breve{\hat{a}} \breve{z}+\nabla \cdot\left(\frac{1}{\nu} \breve{\hat{b}} \breve{z}\right)+\sum_{i=1}^{M} \frac{1}{\nu} \breve{u}_{i} \Phi_{i}=\frac{1}{\nu} \breve{\widehat{N}}(\breve{z}) ;\left.\quad \breve{z}\right|_{\Gamma}=0
$$

which we can do by rescaling time $t=\frac{\tau}{\nu}$ and setting $\breve{p}(\tau):=p\left(\frac{\tau}{\nu}\right)$, for a function $p$ defined for $t \geq 0$.

2.2. The case of boundary controls. As in the internal case, by direct computations we find that $\breve{z}(\tau)=(y-\hat{y})\left(\frac{\tau}{\nu}\right)$ solves

$$
\partial_{\tau} \breve{z}-\Delta \breve{z}+\frac{1}{\nu} \breve{\hat{a}} \breve{z}+\nabla \cdot\left(\frac{1}{\nu} \widehat{\hat{b}} \breve{z}\right)=\frac{1}{\nu} \breve{\widehat{N}}(\breve{z}) ;\left.\quad \breve{z}\right|_{\Gamma}=\sum_{i=1}^{M} \breve{u}_{i} \Psi_{i}
$$

2.3. Stabilization to zero. We can see that our goal (3) is to find the control $u$, in either system (6) or system (7), such that

$$
|\breve{z}(\tau)|_{X}^{2} \leq C \mathrm{e}^{-\bar{\lambda} \tau}|z(0)|_{X}^{2}, \quad \text { provided } \quad|z(0)|_{X}<\epsilon
$$

with $\bar{\lambda}=\frac{\lambda}{\nu}$, for suitable positive constants $C=C_{\bar{\lambda}}$ and $\epsilon=\epsilon_{\bar{\lambda}}$.

We will follow a standard procedure. We will start by proving the global stabilization result for the linearized system, that is, in the case $\breve{\mathcal{N}}=0$. Then, the local stabilization result will follow by a fixed point argument.

\section{INTERNAL STABILIZATION OF THE LINEARIZED SYSTEM}

We consider a system in the form (6), without the nonlinearity. In order to study such system we start by denoting the Hilbert space $H:=L^{2}(\Omega, \mathbb{R})$ which we will consider as a pivot space, $H^{\prime}=H$. We also denote $V:=H_{0}^{1}(\Omega, \mathbb{R})$ and $\mathrm{D}(\Delta):=V \cap H^{2}(\Omega, \mathbb{R})$, which are supposed to be endowed with the scalar products

$$
(v, w)_{V}:=(\nabla v, \nabla w)_{L^{2}\left(\Omega, \mathbb{R}^{d}\right)} \quad \text { and } \quad(v, w)_{\mathrm{D}(\Delta)}:=(\Delta v, \Delta w)_{H},
$$

and corresponding norms $|v|_{V}:=(v, v)_{V}^{\frac{1}{2}}$ and $|v|_{\mathrm{D}(\Delta)}:=(v, v)_{\mathrm{D}(\Delta)}^{\frac{1}{2}}$. 
Moreover we have the inclusions

$$
\mathrm{D}(\Delta) \stackrel{\mathrm{d}, \mathrm{c}}{\longrightarrow} V \stackrel{\mathrm{d}, \mathrm{c}}{\longrightarrow} H \stackrel{\mathrm{d}, \mathrm{c}}{\longrightarrow} V^{\prime} \stackrel{\mathrm{d}, \mathrm{c}}{\longrightarrow} \mathrm{D}(\Delta)^{\prime},
$$

the increasing sequence of repeated eigenvalues $\alpha_{i}, i=1,2, \ldots$, of $-\Delta$ satisfy

$$
0<\alpha_{1}<\alpha_{2} \leq \alpha_{3} \leq \ldots, \quad \lim _{i \rightarrow+\infty} \alpha_{i}=+\infty
$$

and we have

$$
\langle v, w\rangle_{V^{\prime}, V}=(v, w)_{H}, \quad \text { for all } \quad(v, w) \in H \times V .
$$

Boundedness assumption. For $m \in \mathbb{N}_{0}$, in order to simplify the writing we denote

$$
\begin{aligned}
\mathcal{W}^{J} & :=L_{w}^{\infty}\left(J, L^{d}(\Omega, \mathbb{R}) \times L^{\infty}\left(\Omega, \mathbb{R}^{d}\right)\right) \\
\mathcal{W} & :=L_{w}^{\infty}\left(\mathbb{R}_{0}, L^{d}(\Omega, \mathbb{R}) \times L^{\infty}\left(\Omega, \mathbb{R}^{d}\right)\right)
\end{aligned}
$$

where $J \subseteq(0,+\infty)$ is an open interval.

We also fix $a$ and $b$, which may depend on time and space, and a constant $C_{\mathcal{W}} \geq 0$, satisfying

$$
|(a, b)|_{\mathcal{W}}:=\left(|a|_{L^{\infty}\left(\mathbb{R}_{0}, L^{d}\right)}^{2}+|b|_{L_{w}^{\infty}\left(\mathbb{R}_{0}, L^{\infty}\right)}^{2}\right)^{\frac{1}{2}} \leq C_{\mathcal{W}}
$$

Remark 3.1. (A technical measurability detail). The space $L_{w}^{\infty}\left(\mathbb{R}_{0}, L^{\infty}(\Omega, \mathbb{R})\right.$ ) is the Bochner-like notation for $L^{\infty}\left(\mathbb{R}_{0} \times \Omega, \mathbb{R}\right)$, where the subscript $w$ stands for weak measurability. Bochner spaces are usually defined for strongly measurable functions, see Boc33, Section 2]. The Bochner space $L^{\infty}\left((a, b), L^{\infty}(\Omega, \mathbb{R})\right)$ consisting of strongly measurable functions is strictly contained in $L_{w}^{\infty}\left(\mathbb{R}_{0}, L^{\infty}(\Omega, \mathbb{R})\right)=\left(L^{1}\left((a, b), L^{1}(\Omega, \mathbb{R})\right)\right)^{\prime}$, see [Fat99, Example 5.0.10]. This is due to the fact that $L^{\infty}(\Omega, \mathbb{R})$ is not separable. Recall also [Pet38, Theorem 1.1] for a relation between strong and weak measurability. However, the norm in $L_{w}^{\infty}\left((a, b), L^{\infty}(\Omega, \mathbb{R})\right)$ is essentially the usual norm of $L^{\infty}\left((a, b), L^{\infty}(\Omega, \mathbb{R})\right)$, see [Fat99, Lemma 9.1.2 (ii)], [Fat05, Lemma 4.1.1], and the miscellaneous notes at the end of Section 4.1 in [Fat05]. Hereafter, we are going to use some arguments from Rod15a, KR15b, KR15a, whose results should be understood with the subscript $w$ in Rod15a, Equation (2.2) and Remark 2.15], [KR15b, Equation (2.1)], and [KR15a, Equation (5)].

3.1. Weak solutions. Let us consider the interval $I=\left(s_{0}, s_{1}\right)$ with $0 \leq s_{0}<s_{1}$, whose length we denote by $|I|:=s_{1}-s_{0}$. Here we recall some regularity results for the weak solutions for systems as (6). We start considering the more general system

$$
\begin{aligned}
& \partial_{t} z-\Delta z+a z+\nabla \cdot(b z)+f=0, \\
& \left.z\right|_{\Gamma}=0, \quad z(0)=z_{0} .
\end{aligned}
$$

where the control is replaced by a general external force.

Lemma 3.2. We have, for $z \in V$

$$
\begin{aligned}
& \langle a z, z\rangle_{V^{\prime}, V} \leq C|a|_{L^{d}}|z|_{H}|z|_{V}, \quad|a z|_{V^{\prime}} \leq C|a|_{L^{d}}|z|_{H}^{\frac{1}{2}}|z|_{V}^{\frac{1}{2}}, \quad \text { for } \quad d \in\{1,2\} . \\
& \langle a z, z\rangle_{V^{\prime}, V} \leq C|a|_{L^{d}}|z|_{H}|z|_{V}, \quad|a z|_{V^{\prime}} \leq C|a|_{L^{d}}|z|_{H}, \quad \text { for } \quad d \geq 3 . \\
& \langle\nabla \cdot(b z), z\rangle_{V^{\prime}, V} \leq C|b|_{L^{\infty}}|z|_{H}|z|_{V}, \quad|\nabla \cdot(b z)|_{V^{\prime}} \leq C|b|_{L^{\infty}}|z|_{H}, \quad \text { for } \quad d \geq 1 \text {. }
\end{aligned}
$$

for a suitable constant $C \geq 0$, depending only on $(\Omega, d)$.

Proof. Concerning the reaction term, in the case $d=1$, and $\Omega=(l, r)$ with $l<r$, from $\frac{\mathrm{d}}{\mathrm{d} x}|z|_{\mathbb{R}}^{2}=2 z \frac{\mathrm{d}}{\mathrm{d} x} z$ we can see that for $z \in V$ and $s \in \Omega$, since $z(l)=z(r)=0$,

$$
|z(s)|_{\mathbb{R}}^{2}=|z(s)|_{\mathbb{R}}^{2}-|z(l)|_{\mathbb{R}}^{2}=2 \int_{l}^{s} z(\tau) \frac{\mathrm{d}}{\mathrm{d} x} z(\tau) \mathrm{d} \tau \leq 2|z|_{L^{2}(\Omega, \mathbb{R})}\left|\frac{\mathrm{d}}{\mathrm{d} x} z\right|_{L^{2}(\Omega, \mathbb{R})} .
$$


That is, we have the Agmon inequality

$$
|z|_{L^{\infty}} \leq 2^{\frac{1}{2}}|z|_{H}^{\frac{1}{2}}|z|_{V}^{\frac{1}{2}}, \quad \text { for } \quad d=1
$$

Therefore, it follows that

$$
\langle a z, w\rangle_{V^{\prime}, V} \leq|a|_{L^{1}}|z|_{L^{\infty}}|w|_{L^{\infty}} \leq 2|a|_{L^{1}}|z|_{H}^{\frac{1}{2}}|z|_{V}^{\frac{1}{2}}|w|_{H}^{\frac{1}{2}}|w|_{V}^{\frac{1}{2}} .
$$

For the cases $d \geq 2$, we will use suitable Sobolev embeddings (cf.[DD12, Corollary 4.53]). For $d=2$, from $H^{\frac{1}{2}}(\Omega, \mathbb{R}) \hookrightarrow L^{4}(\Omega, \mathbb{R})$ and an interpolation argument (cf. [LM72a, chapter 1, section 9.1]), we find

$$
\langle a z, w\rangle_{V^{\prime}, V} \leq|a|_{L^{2}}|z|_{L^{4}}|w|_{L^{4}} \leq C_{2}|a|_{L^{2}}|z|_{H}^{\frac{1}{2}}|z|_{V}^{\frac{1}{2}}|w|_{H}^{\frac{1}{2}}|w|_{V}^{\frac{1}{2}} .
$$

For $d \geq 3$, from $H^{1}(\Omega, \mathbb{R}) \hookrightarrow L^{\frac{2 d}{d-2}}(\Omega, \mathbb{R})$, we find

$$
\langle a z, z\rangle_{V^{\prime}, V} \leq|a|_{L^{d}}|z|_{L^{\frac{2 d}{d-2}}}|z|_{L^{2}} \leq C_{3}|a|_{L^{d}}|z|_{V}|z|_{H}
$$

Finally, writing $\langle\nabla \cdot(b z), z\rangle_{V^{\prime}, V}=(b z, \nabla z)_{L^{2}\left(\Omega, \mathbb{R}^{d}\right)}$, it will follow the estimates for the convection term.

Lemma 3.3. Given $f \in L^{2}\left(I, V^{\prime}\right)$ and $z_{0} \in H$, there is a weak solution $z \in W\left(I, V, V^{\prime}\right)$ for (10). Moreover $z$ is unique and depends continuously on the data:

$$
|z|_{W\left(I, V, V^{\prime}\right)}^{2} \leq \bar{C}_{\left[|I|, C_{\mathcal{W}}\right]}\left(\left|z\left(s_{0}\right)\right|_{H}^{2}+|f|_{L^{2}\left(I, V^{\prime}\right)}^{2}\right) .
$$

The procedure is well known, yet we will recall some steps of the proof since some estimates from the proof will be used later on.

Weak solutions for system (10) are understood in the variational sense. We will restrict ourselves to the derivation of some a priori (like) estimates. In fact those estimates will also hold for Galerkin approximations of the system, for example using a basis of eigenfunctions of the Laplace operator $\Delta$, thus the estimates can be used to precisely derive the existence of weak solutions. See [Lio69, Chapter 1, Section 6], [Tem95, Chapter 1, Section 3], and [Tem01, Chapter 3, Sections 1.3, 1.4, and 3.2] for more details on the procedure.

We start with the following auxiliary result.

Corollary 3.4. We have, for $z \in V$

$$
\begin{aligned}
& 2\langle a z+\nabla \cdot(b z), z\rangle_{V^{\prime}, V} \leq D_{\mathrm{rc}, 1}|(a, b)|_{\mathcal{W}}^{2}|z|_{H}^{2}+\frac{1}{2}|z|_{V}^{2} \\
& 2\langle a z+\nabla \cdot(b z), z\rangle_{V^{\prime}, V} \leq D_{\mathrm{rc}}|(a, b)|_{\mathcal{W}}^{2}|z|_{H}^{2}+\frac{3}{2}|z|_{V}^{2} .
\end{aligned}
$$

for suitable constants $D_{\mathrm{rc}} \leq D_{\mathrm{rc}, 1}$ depending only on $(\Omega, d)$.

Proof. From Lemma 3.2 , and for any $\alpha>0$, we have $2\langle a z+\nabla \cdot(b z), z\rangle_{V^{\prime}, V} \leq 2 C\left(|a|_{L^{d}}+\right.$ $\left.|b|_{L^{\infty}}\right)|z|_{H}|z|_{V} \leq \frac{1}{\alpha} C^{2}\left(|a|_{L^{d}}+|b|_{L^{\infty}}\right)^{2}|z|_{H}^{2}+\alpha|z|_{V}^{2} \leq \frac{2}{\alpha} C^{2}\left(|a|_{L^{d}}^{2}+|b|_{L^{\infty}}^{2}\right)|z|_{H}^{2}+2 \alpha|z|_{V}^{2}$.

Proof of Lemma 3.3. Multiplying (10a) by $2 z$, formally we find

$$
\frac{\mathrm{d}}{\mathrm{d} t}|z|_{H}^{2}+2|z|_{V}^{2}=2\langle a z+\nabla \cdot(b z), z\rangle_{V^{\prime}, V}+2\langle f, z\rangle_{V^{\prime}, V}
$$

from which, by appropriate Young inequalities, we can obtain

$$
\begin{aligned}
\frac{\mathrm{d}}{\mathrm{d} t}|z|_{H}^{2}+|z|_{V}^{2} & \leq D_{\mathrm{rc}, 1}|(a, b)|_{\mathcal{W}}^{2}|z|_{H}^{2}+2|f|_{V^{\prime}}^{2} \\
\frac{\mathrm{d}}{\mathrm{d} t}|z|_{H}^{2} & \leq D_{\mathrm{rc}}|(a, b)|_{\mathcal{W}}^{2}|z|_{H}^{2}+2|f|_{V^{\prime}}^{2} .
\end{aligned}
$$


By $(12 \mathrm{~b})$ and the Gronwall inequality, it follows that for all $s \in I$

$$
|z(s)|_{H}^{2} \leq \mathrm{e}^{D_{\mathrm{rc}}|(a, b)|_{\mathcal{W}}^{2}\left(s-s_{0}\right)}\left(\left|z\left(s_{0}\right)\right|_{H}^{2}+2|f|_{L^{2}\left(I, V^{\prime}\right)}^{2}\right),
$$

and integrating (12a),

$$
|z(s)|_{H}^{2}+|z|_{L^{2}\left(\left(s_{0}, s\right), V\right)}^{2} \leq\left|z\left(s_{0}\right)\right|_{H}^{2}+D_{\mathrm{rc}, 1}|(a, b)|_{\mathcal{W}}^{2}|z|_{L^{2}(I, H)}^{2}+2|f|_{L^{2}\left(I, V^{\prime}\right)}^{2} .
$$

From 10a and Lemma 3.2 we can also derive

$$
\left|\partial_{t} z\right|_{L^{2}\left(I, V^{\prime}\right)} \leq|z|_{L^{2}(I, V)}+\bar{C}_{\left[C_{\mathcal{W}}\right]}|z|_{L^{2}(I, H)}^{2}+|f|_{L^{2}\left(I, V^{\prime}\right)},
$$

from which, using (13) and (14) we can conclude that

$$
|z|_{W\left(I, V, V^{\prime}\right)}^{2} \leq \bar{C}_{\left[s_{1}-s_{0}, C_{\mathcal{W}}\right]}\left(\left|z\left(s_{0}\right)\right|_{H}^{2}+|f|_{L^{2}\left(I, V^{\prime}\right)}^{2}\right) .
$$

Finally the uniqueness of $z$, follows from the fact that if $\tilde{z}$ is another weak solution, then $e=z-\tilde{z}$, solves $(10)$ with $e\left(s_{0}\right)=0$ and $f=0$. From 113 it will follow that $|e(s)|_{H}=0$ for all $s \in I$.

3.2. Null controllability. Here we recall the relation between null controllability of system (10) and a suitable observability inequality for the adjoint system.

Consider, in the bounded cylinder $I \times \Omega, I=\left(s_{0}, s_{1}\right)$, the controlled system

$$
\begin{aligned}
& \partial_{t} z-\Delta z+a z+\nabla \cdot(b z)+B \eta=0, \\
& \left.z\right|_{\Gamma}=0, \quad z\left(s_{0}\right)=z_{0},
\end{aligned}
$$

where now our control is a function $\eta \in L^{2}(I, H)$ and $B \in \mathcal{L}(H)$ with adjoint denoted by $B^{*}$. Let us also consider in $I \times \Omega$ the adjoint system

$$
\begin{aligned}
& -\partial_{t} q-\Delta q+a q-b \cdot \nabla q=0, \\
& \left.q\right|_{\Gamma}=0, \quad q\left(s_{1}\right)=q_{1} \in H,
\end{aligned}
$$

and let $z\left(z_{0}, \eta\right)(t):=z(t)$ and $q\left(q_{1}\right)(t):=q(t)$ denote the solutions of (15) and (16), for given data $\left(z_{0}, u\right)$ and $q_{1}$, respectively. Notice that, proceeding as in section 3.1, we can prove the existence of weak solutions $q \in W\left(I, V, V^{\prime}\right)$ for system (16).

Definition 3.5. (i) We say that (15) is null controllable in $I$ if there exists a family $\left\{\eta\left(z_{0}\right) \mid z_{0} \in H\right\} \subset L^{2}(I, H)$ such that $z\left(z_{0}, \eta\left(z_{0}\right)\right)\left(s_{1}\right)=0$, for $z_{0} \in H$.

(ii) We say that (16) is $B^{*}$-observable in $I$ if there exists a constant $C_{\text {obs }}>0$ such that for all $q_{1} \in H$ we have that the corresponding weak solution $q$ satisfies the inequality

$$
\left|q\left(q_{1}\right)\left(s_{0}\right)\right|_{H} \leq C_{\text {obs }}\left|B^{*} q\left(q_{1}\right)\right|_{L^{2}(I, H)} .
$$

The constant $C_{\text {obs }}$ in (17) depends, in general, on $\Omega, \omega, I, B$, and also on the coefficient functions $a$ and $b$.

The following lemma, can be proven by a standard procedure. For details, we refer to [AKBGBdT11, Section 2], Cor07, Chapter 2].

Lemma 3.6. System (16) is $B^{*}$-observable in $I$ if, and only if, system (15) is null controllable in $I$ and the family of controls $\left\{\eta\left(z_{0}\right) \mid z_{0} \in H\right\}$ can be chosen as a bounded linear function of $z_{0}$ :

$$
\left|\eta\left(z_{0}\right)\right|_{L^{2}(I, H)} \leq C_{\mathrm{obs}}\left|z_{0}\right|_{H} \text {, where } C_{\mathrm{obs}} \text { is as in (17). }
$$


Controls supported in a subset. Given an open subset $\omega \subseteq \Omega$, then from [DZZ08, Theorem 2.1] and [DFCGBZ02, Theorem 2.3] (e.g., reversing time in system (16)) we have that there exists a constant $C_{\omega, \Omega}>0$, depending on $\omega$ and $\Omega$, such that the weak solution $q$ for (16) satisfies

$$
|q(0)|_{H}^{2} \leq \mathrm{e}^{C_{\omega, \Omega} \Theta\left(|I|,|a|_{L^{\infty}\left(I, L^{d}\right)},|b|_{\left.L_{w}^{\infty}\left(I, L^{\infty}\right), d\right)}\right.}|q|_{L^{2}\left(I, L^{2}(\omega, \mathbb{R})\right.}^{2} .
$$

with

$$
\Theta\left(r, \theta_{1}, \theta_{2}, d\right):=1+\theta_{1}^{2}+d \theta_{2}^{2}+\frac{1}{r}+r\left(\theta_{1}+d \theta_{2}^{2}\right),
$$

for $r>0, \theta_{1} \geq 0 \leq \theta_{2}$, and $d \in \mathbb{N}_{0}$.

Therefore, in the case we take $B=1_{\omega} \in \mathcal{L}(H)$ with

$$
1_{\omega} \eta(x):=\left\{\begin{array}{ll}
\eta(x), & \text { if } x \in \omega \\
0, & \text { if } x \in \Omega \backslash \bar{\omega}
\end{array},\right.
$$

then we have $B^{*}=B$ and $|q|_{L^{2}\left(I, L^{2}(\omega, \mathbb{R})\right)}^{2}=\left|B^{*} q\right|_{L^{2}(I, H)}^{2}$, and we can conclude that (17) holds with $C_{\text {obs }}=\mathrm{e}^{C_{\omega, \Omega} \Theta\left(|I|,|a|_{L^{\infty}\left(I, L^{d}\right)},|b|_{L_{w}^{\infty}\left(I, L^{\infty}\right)}, d\right)}$. Therefore we have the following.

Theorem 3.7. Let $B=1_{\omega}$ and let $I=\left(s_{0}, s_{1}\right)$ be arbitrary, then, there exists a family $\left\{\eta\left(z_{0}\right) \mid z_{0} \in H\right\} \subseteq L^{2}(I, H)$ such that the solutions $z\left(z_{0}, \eta\left(z_{0}\right)\right)$ to (15) satisfy $z\left(z_{0}, \eta\left(z_{0}\right)\right)\left(s_{1}\right)=0$ and, for a constant $\widehat{C}=C(\omega, \Omega)$, we have that

$$
\left|\eta\left(z_{0}\right)\right|_{L^{2}(I, H)} \leq \mathrm{e}^{\widehat{C} \Theta}\left|z_{0}\right|_{H},
$$

with $\Theta=\Theta\left(|I|,|a|_{L^{\infty}\left(\mathbb{R}_{0}, L^{d}\right)},|b|_{L_{w}^{\infty}\left(\mathbb{R}_{0}, L^{\infty}\right)}, d\right)$ given by 18$)$.

Notice that since (17) holds with $C_{\text {obs }}=\mathrm{e}^{\widehat{C} \Theta}$ and $B=1_{\omega}$. Proceeding as in [BRS11, Section A.2] we can conclude that (17) also holds with $C_{\text {obs }}=C_{\chi} \mathrm{e}^{\widehat{C}_{\chi} \Theta} \leq \mathrm{e}^{\widehat{D} \Theta}$ and $B^{*} q:=$ $\chi 1_{\omega} q=1_{\omega} \chi 1_{\omega} q$, where $\widehat{D}=\log \left(C_{\chi}\right)+\widehat{C}_{\chi}$ and $\chi \in C^{\infty}(\bar{\Omega})$ is any given smooth function with $\emptyset \neq \omega \cap \operatorname{supp} \chi$. Here $\widehat{D}=\widehat{D}(\chi, \omega, \Omega)>0$ depends only on $(\chi, \omega, \Omega)$. Notice that $\Theta\left(r, \theta_{1}, \theta_{2}, d\right) \geq 1$.

Corollary 3.8. Theorem 3.7 holds in the more general case $B=1_{\omega} \chi 1_{\omega}$, with $\widehat{D}$ in the place of $\widehat{C}$.

3.3. Stabilization to zero by finite dimensional controls. Here we analyze the case when stabilization of system (15) can be achieved by finite dimensional control action, of the form $\sum_{i=1}^{M} u_{i}(t) \Phi_{i}(x)$. Following the ideas in [KR15b, KR15a, BKR15], we consider a family $\widehat{\mathcal{C}}_{\omega}=\left\{\widehat{\Phi}_{i} \in H \mid i \in\{1,2, \ldots, M\}\right\} \subset H$ and denote by $P_{M}$ the orthogonal projection in $H$ onto $\mathcal{S}_{\widehat{\mathcal{C}}_{\omega}}:=\operatorname{span} \widehat{\mathcal{C}}_{\omega}$.

Let us also fix a positive constant $\bar{\lambda}>0$ and consider, in $\mathbb{R}_{s_{0}} \times \Omega$, the system:

$$
\begin{aligned}
& \partial_{t} z-\Delta z+a z+\nabla \cdot(b z)+1_{\omega} \chi P_{M} 1_{\omega} \eta=0, \\
& \left.z\right|_{\Gamma}=0, \quad z\left(s_{0}\right)=z_{0} .
\end{aligned}
$$

Definition 3.9. We say that $(19)$ is exponentially stabilizable to zero, with rate $\frac{\bar{\lambda}}{2}$, if there are a constant $C>0$ and a family $\left\{\eta=\eta\left(z_{0}\right) \mid z_{0} \in H\right\} \subseteq L^{2}\left(\mathbb{R}_{s_{0}}, H\right)$ such that the corresponding global solution $z(t)=z\left(z_{0}, \eta\left(z_{0}\right)\right)(t)$ satisfies

$$
|z(t)|_{H}^{2} \leq C \mathrm{e}^{-\bar{\lambda}\left(t-s_{0}\right)}\left|z_{0}\right|_{H}^{2}, \quad \text { for all } t \geq s_{0} .
$$


Notice that the stabilizing control in $19 \mathrm{a}$ takes its values in the finite dimensional space $1_{\omega} \chi \mathcal{S}_{\mathcal{C}_{\omega}}=\operatorname{span}\left\{1_{\omega} \chi \widehat{\Phi}_{i} \in H \mid i \in\{1,2, \ldots, M\}\right\}$, for all $t \in \mathbb{R}_{s_{0}}$.

$$
1_{\omega} \chi P_{M} 1_{\omega} \eta=1_{\omega} \chi \sum_{i=1}^{M} \eta_{i} \widehat{\Phi}_{i}=\sum_{i=1}^{M} u_{i} \Phi_{i}
$$

with $u_{i}:=\eta_{i}$ and $\Phi_{i}:=1_{\omega} \chi \widehat{\Phi}_{i}, i \in\{1,2, \ldots, M\}$.

Remark 3.10. Without loss of generality we can suppose that the family $\widehat{\mathcal{C}}_{\omega}$ is linearly independent and orthonormal. In that case $\eta_{i}(t)=\left(\eta(t, \cdot), \widehat{\Phi}_{i}\right)_{H}$.

In the following, the function $\Theta$ and the constant $\widehat{D}$ are as in Theorem 3.7 and Corollary 3.8. Let $\chi \in C^{\infty}(\bar{\Omega})$ satisfy $\emptyset \neq \omega \cap \operatorname{supp} \chi$, and consider the system

$$
\begin{aligned}
& \partial_{t} z-\Delta z+\left(a-\frac{\bar{\lambda}}{2}\right) z+\nabla \cdot(b z)+1_{\omega} \chi 1_{\omega} \eta=0, \\
& \left.z\right|_{\Gamma}=0, \quad z\left(s_{0}\right)=z_{0} .
\end{aligned}
$$

Lemma 3.11. Let $I=\left(s_{0}, s_{1}\right)$. The solution of system $(20)$, in $I \times \Omega$, with the control $\eta$ given by Corollary 3.8, satisfies

$$
\begin{aligned}
& \left|z\left(s_{0}+\tau\right)\right|_{H}^{2} \leq \mathrm{e}^{D_{\mathrm{rc}}\left|\left(a-\frac{\bar{\lambda}}{2}, b\right)\right|_{\mathcal{W}^{\tau}}^{2}}\left|z\left(s_{0}\right)\right|_{H}^{2} \\
& +2|\iota|_{\mathcal{L}\left(H, V^{\prime}\right)}^{2} \mathrm{e}^{D_{\mathrm{rc}}\left|\left(a-\frac{\bar{\lambda}}{2}, b\right)\right|_{\mathcal{W}^{2}}^{2} \tau+\widehat{D} \Theta\left(|I|,|a|_{L^{\infty}\left(\mathbb{R}_{0}, L^{d}\right)},|b|_{L_{w}^{\infty}\left(\mathbb{R}_{0}, L \infty\right)}, d\right)}\left|z\left(s_{0}\right)\right|_{H}^{2},
\end{aligned}
$$

for all $\tau \in I$, where $\iota: H \rightarrow V^{\prime}$ stands for the inclusion mapping $\iota z:=z$.

Proof. Straightforward, from (13). Notice that Corollary 3.8 still holds true with $a-\frac{\bar{\lambda}}{2}$ in the role of $a$.

Inspired by Lemma 3.11, and the procedure in [BKR15, KR15a], we consider the function $\Xi:(0,+\infty) \rightarrow(0,+\infty)$ defined by

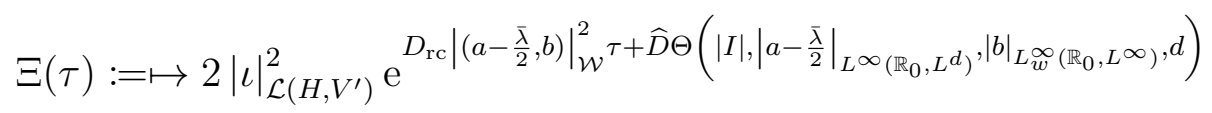

which we can extend to a function $\Xi^{\mathrm{ex}}:[0,+\infty] \rightarrow(0,+\infty]$, by setting

$$
\Xi^{\operatorname{ex}}(\tau):= \begin{cases}\Xi(\tau), & \text { if } \tau \in(0,+\infty) \\ \lim _{t \rightarrow \tau} \Xi(t), & \text { if } \tau \in\{0,+\infty\} .\end{cases}
$$

The minimum and minimizer of $\Xi^{\mathrm{ex}}$ are denoted by $\Upsilon$ and $T_{*}$, respectively. From

$$
\frac{\left.\frac{\mathrm{d} \Xi}{\mathrm{d} \tau}\right|_{\tau=t}}{\Xi(t)}=\left(D_{\mathrm{rc}}\left|\left(a-\frac{\bar{\lambda}}{2}, b\right)\right|_{\mathcal{W}}^{2}+\widehat{D}\left(-t^{-2}+\left|a-\frac{\bar{\lambda}}{2}\right|_{L^{\infty}\left(\mathbb{R}_{0}, L^{d}\right)}+d|b|_{L_{w}^{\infty}\left(\mathbb{R}_{0}, L^{\infty}\right)}^{2}\right)\right),
$$

we can conclude that $T_{*}>0$ can be defined by

$$
T_{*}^{2}=\frac{\widehat{D}}{D_{\mathrm{rc}}\left|\left(a-\frac{\bar{\lambda}}{2}, b\right)\right|_{\mathcal{W}}^{2}+\widehat{D}\left(\left|a-\frac{\bar{\lambda}}{2}\right|_{L^{\infty}\left(\mathbb{R}_{0}, L^{d}\right)}+d|b|_{L_{w}^{\infty}\left(\mathbb{R}_{0}, L^{\infty}\right)}^{2}\right)} .
$$

Further $T_{*}=+\infty$ if, and only if, both $a-\frac{\bar{\lambda}}{2}$ and $b$ vanish.

Thus, if $T_{*} \in \mathbb{R}_{0}$, we have that the minimum $\Upsilon=\Xi^{\operatorname{ex}}\left(T_{*}\right)$ is given by

$$
\Upsilon=2|\iota|_{\mathcal{L}\left(H, V^{\prime}\right)}^{2} \mathrm{e}^{\bar{\Theta}\left(\left|a-\frac{\bar{\lambda}}{2}\right|_{L^{\infty}\left(\mathbb{R}_{0}, L^{d}\right)},|b|_{L_{w}^{\infty}\left(\mathbb{R}_{0}, L^{\infty}\right)},\left|\left(a-\frac{\bar{\lambda}}{2}, b\right)\right|_{\mathcal{W}^{\prime}}, d\right)},
$$


and, if $T_{*}=+\infty$ by

$$
\Upsilon=2|\iota|_{\mathcal{L}\left(H, V^{\prime}\right)}^{2} \mathrm{e}^{\widehat{D}}
$$

where

$$
\bar{\Theta}\left(\xi_{1}, \xi_{2}, \xi_{3}, d, \nu\right):=\widehat{D}\left(1+\xi_{1}^{2}+d \xi_{2}^{2}\right)+2(\widehat{D})^{\frac{1}{2}}\left(D_{\mathrm{rc}} \xi_{3}^{2}+\widehat{D}\left(\xi_{1}+d \xi_{2}^{2}\right)\right)^{\frac{1}{2}} .
$$

The following result gives us a sufficient condition on the family $\widehat{\mathcal{C}_{\omega}}$ for the existence of a stabilizing control. The proof can be done following the arguments in [KR15a, BKR15].

Theorem 3.12. Let us be given $\chi \in C^{\infty}(\bar{\Omega})$ satisfying $\emptyset \neq \omega \cap \operatorname{supp} \chi$. If

$$
T_{*} \in \mathbb{R}_{0} \quad \text { and } \quad\left|1_{\omega} \chi\left(1-P_{M}\right) 1_{\omega}\right|_{\mathcal{L}\left(H, V^{\prime}\right)}^{2} \leq \Upsilon^{-1}
$$

with $T_{*}$ as in (21) and $\Upsilon$ as in (22a), then system (19) is stabilizable to zero with rate $\frac{\bar{\lambda}}{2}$. Moreover, we can set the stabilizing control function $\eta=\eta\left(z_{0}\right)$ such that

$$
\begin{aligned}
|z(t)|_{H}^{2} & \leq\left(\Upsilon_{0}+\Upsilon\left|B_{M}\right|_{\mathcal{L}\left(H, V^{\prime}\right)}^{2}\right) \mathrm{e}^{-\bar{\lambda}\left(t-s_{0}\right)}\left|z_{0}\right|_{H}^{2}, \text { for } t \geq s_{0}, \\
\left|\mathrm{e}^{\frac{\hat{\lambda}}{2}} \cdot \eta\left(z_{0}\right)\right|_{L^{2}\left(\mathbb{R}_{s_{0}}, H\right)}^{2} & \leq \frac{1}{1-\mathrm{e}^{(\hat{\lambda}-\bar{\lambda}) T_{*}}} \mathrm{e}^{2 \widehat{D} \Theta_{*}}\left|z_{0}\right|_{H}^{2}, \text { for } \hat{\lambda}<\bar{\lambda}
\end{aligned}
$$

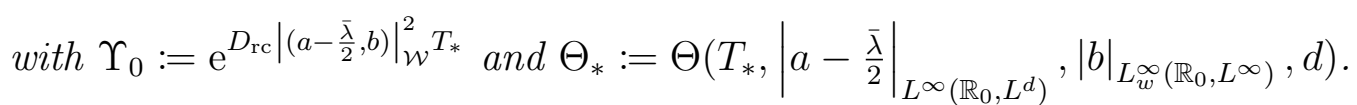

If $T_{*}=+\infty$, then setting $\eta=\eta\left(z_{0}\right)=0$ the solution $z$ of system (19) satisfies $|z(t)|_{H}^{2} \leq$ $\mathrm{e}^{-\bar{\lambda}\left(t-s_{0}\right)}\left|z_{0}\right|_{H}^{2}$, for $t \geq s_{0}$.

3.4. The dimension of the control. Families $\widehat{\mathcal{C}}_{\omega}$ which satisfy 24 do exist, for example in the case the control domain is an open nonempty rectangle $\omega=\omega_{R}:=$ $\prod_{j=1}^{n}\left(l_{j, 1}, l_{j, 2}\right) \subset \Omega$. We give two examples

Ex1. $0 \neq \chi \in C^{\infty}(\bar{\Omega})$ such that $\operatorname{supp} \chi \subseteq \overline{\omega_{R}}$ and $\widehat{\mathcal{C}}_{\omega}:=\left\{\widehat{\Psi}_{R, i} \mid i \in\{1,2, \ldots, M\}\right\}$ for big enough $M$, where $\left\{\widehat{\Psi}_{R, i} \mid i \in \mathbb{N}_{0}\right\}$ is a complete system of eigenfunctions of the negative Laplacian $-\Delta$ in $\omega_{R}$ with homogeneous Dirichlet boundary conditions, which are ordered according to the increasing sequence of the (repeated) eigenvalues: $0<\bar{\lambda}_{i} \leq \bar{\lambda}_{i+1}$, $\lim _{i \rightarrow \infty} \bar{\lambda}_{i}=\infty$.

Ex2. $\chi=\mathbb{1}_{\Omega}$ and we consider a family $\widehat{\mathcal{C}}_{\omega}=\left\{\Phi_{i}=\mathbb{1}_{R_{i}} \mid i \in\{1,2, \ldots, M\}\right\} \in H$ where the $R_{i} s$ are the sub-rectangles in a uniform partition of $\omega_{R}$, and

$$
\mathbb{1}_{\mathcal{O}}(x):=\left\{\begin{array}{ll}
1, & \text { if } x \in \mathcal{O}, \\
0, & \text { if } x \in \Omega \backslash \overline{\mathcal{O}},
\end{array} \quad \text { for an open subset } \quad \mathcal{O} \subseteq \Omega .\right.
$$

That is, each interval $\left(l_{j, 1}, l_{j, 2}\right)$ is divided into $p_{j}$ intervals: $I_{j, k}=\left(l_{j, 1}+k_{j} \frac{\bar{l}_{j}}{p_{j}}, l_{j, 1}+\left(k_{j}+\right.\right.$ 1) $\left.\frac{\bar{l}_{j}}{p_{j}}\right)$, with $k_{j} \in\left\{0,1, \ldots, p_{j}-1\right\}$ and $\bar{l}_{j}:=l_{j, 2}-l_{j, 1}$. In this way, our rectangle is divided into $M=\prod_{j=1}^{d} p_{j}$ sub-rectangles $\left\{R_{i} \mid i \in\{1,2, \ldots, M\}\right\}=\left\{\prod_{j=1}^{n} I_{j, k_{j}} \mid k_{j} \in\right.$ $\left.\left\{0,1, \ldots, p_{j}-1\right\}\right\}$.

Proceeding as in [BKR15, Example 2.14], we can see that in the case of example Ex1 above, condition (24) is satisfied provided that $4|\chi|_{C^{1}(\bar{\Omega})}^{2}\left(\bar{\lambda}_{M}+1\right)^{-1} \leq \Upsilon^{-1}$, that is, provided $\bar{\lambda}_{M}+1 \geq 4|\chi|_{C^{1}(\bar{\Omega})}^{2} \Upsilon$. Furthermore, from [LY83, Corollary 1] we have the asymptotic behaviour $\bar{\lambda}_{M} \geq D_{d} M^{\frac{2}{d}}$ with

$$
D_{d}=\frac{4 d \pi^{2}}{(d+2)\left|w_{R}\right|^{\frac{2}{d}}\left|\mathcal{B}_{d}\right|^{\frac{2}{d}}},
$$


where $\left|w_{R}\right|=\prod_{j=1}^{d} \bar{l}_{j}$ is the volume of $w_{R}$ and $\left|\mathcal{B}_{d}\right|$ is the volume of the unit ball $\mathcal{B}_{d}:=$ $\left\{\left.x \in \mathbb{R}^{d}|| x\right|_{\mathbb{R}^{d}} \leq 1\right\}$, we can also arrive at the sufficient condition

$$
M \geq D_{d}^{-\frac{d}{2}}\left(4|\chi|_{C^{1}(\bar{\Omega})}^{2} \Upsilon\right)^{\frac{d}{2}}
$$

which gives us an upper bound on the number $M$ of actuators which are needed to stabilize the system.

We recall that $\left|\mathcal{B}_{1}\right|=2,\left|\mathcal{B}_{2}\right|=\pi$, and $\left|\mathcal{B}_{3}\right|=\frac{4}{3} \pi$, and in general $\left|\mathcal{B}_{2 k}\right|=\frac{\pi^{k}}{\Gamma(k+1)}=\frac{\pi^{k}}{k !}$ and also $\left|\mathcal{B}_{2 k+1}\right|=\frac{\pi^{k+\frac{1}{2}}}{\Gamma\left(k+\frac{3}{2}\right)}=\frac{2(k !)(4 \pi)^{k}}{(2 k+1) !}, k \in \mathbb{N}$, where $\Gamma$ stands for the Euler gamma function. See [Fol01, Corollary] and [Bir13, Eq. (10)]. Notice that we can prove the last identity by induction using $\Gamma\left(k+\frac{3}{2}\right)=\Gamma\left(k+\frac{1}{2}+1\right)=\left(k+\frac{1}{2}\right) \Gamma\left((k-1)+\frac{3}{2}\right)$ and $\Gamma\left(\frac{1}{2}\right)=\pi^{\frac{1}{2}}$.

Proceeding as in [BKR15, Example 2.15], we can see that in the case of example Ex2, condition (24) is satisfied provided that the partition is fine enough, so that $\left(\mu_{M} \pi^{2}\right)^{-1} \leq$ $\Upsilon^{-1}$ where $\mu_{M}=\min \left\{\frac{p_{j}^{2}}{l_{j}^{2}} \mid j \in\{1,2, \ldots, M\}\right\}$. Notice that $\mu_{M} \pi^{2}$ is the smallest nonzero eigenvalue of the (negative) Neumann Laplacian in each rectangle $R_{j}$. Thus (24) holds provided $\frac{p^{2}}{\bar{l}^{2}} \geq \frac{\Upsilon}{\pi^{2}}$, where $\underline{p}:=\min \left\{p_{j} \mid j \in\{1,2, \ldots, M\}\right\}$ and $\bar{l}:=\max \left\{\bar{l}_{j} \mid j \in\right.$ $\{1,2, \ldots, M\}\}$. Then we conclude that $\frac{M^{2}}{l^{2 d}} \geq \frac{\Upsilon^{d}}{\pi^{2 d}}$ is sufficient for (24), and thus so is

$$
M \geq\left(\frac{\bar{l}^{2}}{\pi^{2}} \Upsilon\right)^{\frac{d}{2}}
$$

Notice that, from (22) we can see that the above estimates for $M$ depend exponentially on $\left|a-\frac{\bar{\lambda}}{2}\right|_{\mathcal{W}}$ and $|b|_{\mathcal{W}^{d}}$. In the one dimensional case, in [KR15b] it is conjectured that a better estimate might be possible (depending polynomially in $\left|a-\frac{\bar{\lambda}}{2}\right|_{\mathcal{W}}$ and $|b|_{\mathcal{W}^{d}}$, like as in (28) below), and this conjecture is supported from the results of some numerical simulations. Later on, we will present some further simulations, for the two dimensional case, that again support this conjecture.

The particular case $\omega=\Omega$. Proceeding as in [KR15b, section 3.1] we can see that in case there is no constraint in the support of the control, and we take $\chi=\mathbb{1}_{\Omega}$, then we can find a better estimate.

Theorem 3.13. Let $(a, b) \in \mathcal{W}$ and $\bar{\lambda}>0$. Let us also take $\chi=\mathbb{1}_{\Omega}$ and a family $\widehat{\mathcal{C}}_{\omega}:=\left\{\widehat{\Psi}_{i} \mid i \in\{1,2, \ldots, M\}\right\}$, where $\left\{\widehat{\Psi}_{i} \mid i \in \mathbb{N}_{0}\right\}$ is a complete system of eigenfunctions of the negative Laplacian in $\Omega$ ordered as in example Ex1 above. If we take

$$
M \geq\left(\frac{D_{\mathrm{rc}} e^{1}(d+2)}{d \pi^{2}}\right)^{\frac{d}{2}}|\Omega|\left|\mathcal{B}_{d}\right|\left|\left(a-\frac{\bar{\lambda}}{2}, b\right)\right|_{\mathcal{W}}^{d},
$$

then for any given $z_{0} \in H$, there is a control $\eta=\eta^{\bar{\lambda}, a, b}\left(s_{0}, z_{0}\right) \in L^{2}\left(\mathbb{R}_{s_{0}}, H\right)$ such that the corresponding solution $z$ of system (19), satisfies the inequality

$$
|z(t)|_{H}^{2} \leq 2\left(\mathrm{e}^{\frac{1}{2}}+1\right) \mathrm{e}^{-\bar{\lambda}\left(t-s_{0}\right)}\left|z_{0}\right|_{H}^{2}, \quad t \geq 0 .
$$

Furthermore,

$$
\left|\mathrm{e}^{\frac{\widehat{\lambda}}{2}\left(\cdot-s_{0}\right)} \eta^{\bar{\lambda}, a, b}\left(s_{0}, z_{0}\right)\right|_{L^{2}\left(\mathbb{R}_{s_{0}}, H\right)}^{2} \leq \frac{\mathrm{e}^{\frac{1}{2}}}{T_{*}(\bar{\lambda}-\widehat{\lambda})}\left|z_{0}\right|_{H}^{2}, \text { for all } \hat{\lambda}<\bar{\lambda}
$$

Proof. We may proceed as in [KR15b, Section 3.1]. We just recall the main steps. The main idea is to find a suitable time $T_{*}>0$, such that for all $\bar{s}_{0} \geq 0$ we can find a control defined in $\left(\bar{s}_{0}, \bar{s}_{0}+T_{*}\right)$ such that at time $\bar{s}_{0}+T_{*}>0$ we have $\left|\mathrm{e}^{\frac{\bar{\lambda}}{2} T_{*}} z\left(\bar{s}_{0}+T_{*}\right)\right|_{H} \leq\left|z\left(\bar{s}_{0}\right)\right|_{H}$. 
For a given forcing $f$, let us consider the system

$$
\partial_{t} y-\Delta y+\left(a-\frac{\bar{\lambda}}{2}\right) y+\nabla \cdot(b y)+f=0,\left.\quad y\right|_{\Gamma}=0, \quad y\left(\bar{s}_{0}\right)=y_{0} .
$$

Let $w$ solve (31) in the time interval $\left(\bar{s}_{0}, \bar{s}_{0}+T\right)$ with $f=0$. Then, $\delta(t):=\frac{\bar{s}_{0}+T-t}{T} w(t)$, $t \in\left[\bar{s}_{0}, \bar{s}_{0}+T\right]$, solves (31) with $f=\frac{1}{T} w$. Let $\delta_{M}$ solve (31) with $f=\frac{1}{T} P_{M} w$ where $P_{M}$ stands for the orthogonal projection in $H$ onto the space $\operatorname{span}\left\{\widehat{\Psi}_{i} \mid i \in\{1,2, \ldots, M\}\right\}$ spanned by the first eigenfunctions of the Dirichlet Laplacian in $\Omega$.

It follows that $d=\delta-\delta_{M}$ solves (31) with $f=\frac{1}{T}\left(1-P_{M}\right) w$ and by (13) it follows that for all $s \in\left[\bar{s}_{0}, \bar{s}_{0}+T\right]$

$$
\begin{aligned}
|d(s)|_{H}^{2} & \leq \frac{2}{T^{2}} \mathrm{e}^{D_{\mathrm{rc}}\left|\left(a-\frac{\bar{\lambda}}{2}, b\right)\right|_{\mathcal{W}^{T}}^{2}}\left|\left(1-P_{M}\right)\right|_{\mathcal{L}\left(H, V^{\prime}\right)}^{2}|w|_{L^{2}\left(\left(\bar{s}_{0}, \bar{s}_{0}+T\right), H\right)}^{2} \\
& \leq \frac{2}{T} \mathrm{e}^{2 D_{\mathrm{rc}}\left|\left(a-\frac{\bar{\lambda}}{2}, b\right)\right|_{\mathcal{W}^{T}}^{2}}\left|\left(1-P_{M}\right)\right|_{\mathcal{L}\left(H, V^{\prime}\right)}^{2}\left|y_{0}\right|_{H}^{2}
\end{aligned}
$$

Therefore, setting $T=T_{*}$ minimizing the right hand side, that is, setting

$$
T_{*}=\left(2 D_{\mathrm{rc}}\left|\left(a-\frac{\bar{\lambda}}{2}, b\right)\right|_{\mathcal{W}}^{2}\right)^{-1}
$$

we find

$$
|d(s)|_{H}^{2} \leq 4 D_{\mathrm{rc}} \mathrm{e}^{1}\left|\left(a-\frac{\bar{\lambda}}{2}, b\right)\right|_{\mathcal{W}}^{2}\left|\left(1-P_{M}\right)\right|_{\mathcal{L}\left(H, V^{\prime}\right)}^{2}\left|y_{0}\right|_{H}^{2}, \quad s \in\left[\bar{s}_{0}, \bar{s}_{0}+T_{*}\right] .
$$

Hence, we will have

$$
\left|\delta_{M}\left(\bar{s}_{0}+T_{*}\right)\right|_{H}^{2} \leq\left|y_{0}\right|_{H}^{2}
$$

provided

$$
\left|\left(1-P_{M}\right)\right|_{\mathcal{L}\left(H, V^{\prime}\right)}^{-2} \geq 4 D_{\mathrm{rc}} \mathrm{e}^{1}\left|\left(a-\frac{\bar{\lambda}}{2}, b\right)\right|_{\mathcal{W}}^{2}
$$

Thus, from $\left|\left(1-P_{M}\right)\right|_{\mathcal{L}\left(H, V^{\prime}\right)}^{2}=\alpha_{M+1}^{-1}$ and [LY83, Corollary 1], see 25), we have that (32) follows from

$$
\frac{4 d \pi^{2}}{(d+2)|\Omega|^{\frac{2}{d}}\left|\mathcal{B}_{d}\right|^{\frac{2}{d}}} M^{\frac{2}{d}} \geq 4 D_{\mathrm{rc}} \mathrm{e}^{1}\left|\left(a-\frac{\bar{\lambda}}{2}, b\right)\right|_{\mathcal{W}}^{2}
$$

that is, (32) follows from (28).

By 13 and (32), we can also obtain

$$
\begin{aligned}
\left|\delta_{M}(s)\right|_{H}^{2} & \leq 2|\delta(s)|_{H}^{2}+2|d(s)|_{H}^{2} \leq 2|w(s)|_{H}^{2}+2|d(s)|_{H}^{2} \\
& \leq 2 \mathrm{e}^{D_{\mathrm{rc}}\left|\left(a-\frac{\bar{\lambda}}{2}, b\right)\right|_{\mathcal{W}^{T}}^{T_{*}}}\left|z_{0}\right|_{H}^{2}+2\left|z_{0}\right|_{H}^{2}=2\left(\mathrm{e}^{\frac{1}{2}}+1\right)\left|y_{0}\right|_{H}^{2}, \quad s \in\left[\bar{s}_{0}, \bar{s}_{0}+T_{*}\right] .
\end{aligned}
$$

We observe that the control $\eta_{M}:=\frac{1}{T} w$ associated with $\delta_{M}$ satisfies

$$
\left|\eta_{M}\left(\bar{s}_{0}, y_{0}\right)\right|_{L^{2}\left(\left(s_{0}, s_{0}+T_{*}\right), H\right)}^{2} \leq \frac{1}{T_{*}} \mathrm{e}^{\frac{1}{2}}\left|y_{0}\right|_{H}^{2} .
$$

We also see that $T_{*}$ does not depend on $\bar{s}_{0}$. Then, for any given $z_{0} \in H$, we can define the concatenated control $\widetilde{\eta}=\widetilde{\eta}^{\bar{\lambda}, a, b}\left(s_{0}, z_{0}\right)$ in the half line $\mathbb{R}_{s_{0}}=\left(s_{0},+\infty\right)$ time interval as follows

$$
\left\{\begin{array}{l}
\left.\widetilde{\eta}\right|_{\left(s_{0}, s_{0}+T_{*}\right)}=\eta_{M}\left(s_{0}, z_{0}\right), \\
\left.\widetilde{\eta}\right|_{\left(s_{0}+i T_{*}, s_{0}+(i+1) T_{*}\right)}=\eta_{M}\left(s_{0}+i T_{*}, y\left(z_{0},\left.\widetilde{\eta}\right|_{\left(s_{0}, s_{0}+i T_{*}\right)}\right)\left(s_{0}+i T_{*}\right)\right), \quad i \in \mathbb{N}_{0},
\end{array}\right.
$$

where $y\left(z_{0},\left.\widetilde{\eta}\right|_{\left(s_{0}, s_{0}+i T_{*}\right)}\right)(t)$ stands for the solution of 31 , with the setting $\left(\bar{s}_{0}, y_{0}, f\right)=$ $\left(s_{0}, z_{0},\left.\widetilde{\eta}\right|_{\left(s_{0}, s_{0}+i T_{*}\right)}\right)$. 
In this way we have that

$$
\begin{aligned}
& \left|y\left(s_{0}+i T_{*}\right)\right|_{H}^{2} \leq\left|y\left(s_{0}\right)\right|_{H}^{2}, \\
& \text { for all } i \in \mathbb{N} \text {, } \\
& \left|y\left(s_{0}+i T_{*}+\tau\right)\right|_{H}^{2} \leq 2\left(\mathrm{e}^{\frac{1}{2}}+1\right)\left|y\left(s_{0}\right)\right|_{H}^{2}, \\
& \text { for all } \tau \in\left[0, T_{*}\right] \text {, } \\
& |\widetilde{\eta}|_{L^{2}\left(\left(s_{0}+i T_{*}, s_{0}+(i+1) T_{*}\right), H\right)}^{2} \leq \frac{1}{T_{*}} \mathrm{e}^{\frac{1}{2}}\left|y\left(s_{0}\right)\right|_{H}^{2}, \\
& \text { for all } i \in \mathbb{N} \text {. }
\end{aligned}
$$

Finally, we can conclude $(29)$ and $(30)$ from the fact that $z=\mathrm{e}^{-\frac{\bar{\lambda}}{2}\left(\cdot-s_{0}\right)} y$ solves 19 ) in $\mathbb{R}_{s_{0}}$ with $\chi=1$ and $\eta^{\bar{\lambda}, a, b}=\mathrm{e}^{-\frac{\bar{\lambda}}{2}\left(\cdot-s_{0}\right)} \widetilde{\eta}$.

Also, concerning the example $\mathbf{E x 2}$ above, again in the case $\omega=\Omega$ and $\chi=1$, an estimate similar to (28) will follow, for example, in the case $\Omega$ is convex and we take piecewise constant controls related to a partition of $\Omega=\bigcup_{i=1}^{M} \Omega_{j}$ into suitable small convex sub-domains $\Omega_{i}$. Now, if $D$ is the diameter of $\Omega$ we can cover $\Omega$ with $n^{d}$ copies (obtained by suitable translations) of the rectangle $\prod_{i=1}^{d}[0, l]$, with $l=\frac{D}{n}$, and whose diameter is $d^{\frac{1}{2}} l$. Taking the intersection of these rectangle copies with $\Omega$ we still have that we can cover $\Omega$ with $M \leq n^{d}$ convex domains $\left\{\Omega_{i} \mid i \in\{1,2, \ldots, M\}\right\}$, each with diameter not bigger than $d^{\frac{1}{2}} l$. Proceeding as in the proof of Theorem 3.13 we arrive to the sufficient condition (32) for stabilization

$$
\left|\left(1-P_{M}\right)\right|_{\mathcal{L}\left(H, V^{\prime}\right)}^{-2} \geq 4 D_{\mathrm{rc}} \mathrm{e}^{1}\left|\left(a-\frac{\bar{\lambda}}{2}, b\right)\right|_{\mathcal{W}}^{2} .
$$

Now we follow [KR15a, Section IV.A] and [BKR15, Section 2.3, Example 2.15] to compute $\left|\left(1-P_{M}\right)\right|_{\mathcal{L}\left(H, V^{\prime}\right)}^{-2}$. Let us set $\mathcal{C}=\left\{\Psi_{i}=\frac{1}{\left|1_{\Omega_{i}}\right|_{H}} 1_{\Omega_{i}} \mid i \in\{1,2, \ldots, M\} \in H\right.$, $\omega=\Omega$, and $\chi=1$. For given $v \in V$ and $z \in H$ we find that

$$
\begin{aligned}
& \left(1_{\omega}\left(1-P_{M}\right) 1_{\omega} z, v\right)_{V^{\prime}, V}=\left(\left(1-P_{M}\right) z, v\right)_{V^{\prime}, V}=\left(z,\left(1-P_{M}\right) v\right)_{H} \\
= & \left(z, v-\sum_{i=1}^{M}\left(v, \Psi_{i}\right)_{H} \Psi_{i}\right)_{H}=\sum_{i=1}^{M}\left(\left.z\right|_{\Omega_{i}}, \varphi_{i}\right)_{L^{2}\left(\Omega_{i}\right)},
\end{aligned}
$$

where $\varphi_{i}:=\left.v\right|_{\Omega_{i}}-\left.\left(\left.v\right|_{\Omega_{i}},\left.\Psi_{i}\right|_{\Omega_{i}}\right)_{L^{2}\left(\Omega_{i}\right)} \Psi_{i}\right|_{\Omega_{i}}=\left.v\right|_{\Omega_{i}}-\frac{1}{|1|_{L^{2}\left(\Omega_{i}\right)}^{2}}\left(\left.v\right|_{\Omega_{i}}, 1\right)_{L^{2}\left(\Omega_{i}\right)}$ has zero average in $\Omega_{i}$. This allows to conclude that $\left|\nabla \varphi_{i}\right|_{L^{2}\left(\Omega_{i}\right)^{n}}^{2} \geq \mu_{1, i}\left|\varphi_{i}\right|_{L^{2}\left(\Omega_{i}\right)}^{2}$, where $\mu_{1, i}$ is the first nonzero eigenvalue $\mu_{1, i}$ of the Neumann Laplacian in $\Omega_{j}$. Recall that in this case, from [PW60, Equation (1.9)], we know that $\mu_{1, i} \geq \frac{\pi^{2}}{l^{2} d}$. Thus, we find for $z \in H$ and $v \in V$ with $|z|_{H}=1,|v|_{V}=1$ the estimates

$$
\left(1_{\omega}\left(1-P_{M}\right) 1_{\omega} z, v\right)_{V^{\prime}, V} \leq\left.\left.\sum_{i=1}^{M} \mu_{1, i}^{-\frac{1}{2}}|z|_{\Omega_{i}}\right|_{L^{2}\left(\Omega_{i}\right)}|\nabla v|_{\Omega_{i}}\right|_{L^{2}\left(\Omega_{i}\right)} \leq d^{\frac{1}{2}} l \pi^{-1} .
$$

Therefore $\left|\left(1-P_{M}\right)\right|_{\mathcal{L}\left(H, V^{\prime}\right)}^{2} \leq l^{2} \pi^{-2} d$, and we can conclude that

$$
l^{-2} \geq 4 \pi^{-2} d D_{\text {rc }} \mathrm{e}^{1}\left|\left(a-\frac{\bar{\lambda}}{2}, b\right)\right|_{\mathcal{W}}^{2}
$$

is a sufficient condition for stabilization. That is, the $M \leq n^{d}=D^{d} l^{-d}$ piecewise constant actuators in $\mathcal{C}$ above are able to stabilize the system. That is, it is enough to take

$$
M \geq\left(\frac{4 D_{\mathrm{rc}} \mathrm{e}^{1}}{\pi^{2}}\right)^{\frac{d}{2}} D^{d} d^{\frac{d}{2}}\left|\left(a-\frac{\bar{\lambda}}{2}, b\right)\right|_{\mathcal{W}}^{d}
$$

piecewise constant actuators as above to stabilize the system. 
3.5. Feedback stabilizing rule and Riccati equation. From Theorem 3.12 we know that system (19) is stabilizable with rate $\frac{\bar{\lambda}}{2}$, provided (24) holds true. Here we recall that in that case the control can be taken in feedback form, i.e.

$$
\eta=\mathcal{F}_{\bar{\lambda}}(t) z=B_{M}^{*} \Pi_{\bar{\lambda}}(t) z
$$

with

$$
B_{M}:=1_{\omega} \chi P_{M} 1_{\omega}
$$

Actually, the procedure is standard. We have just to follow the arguments in BRS11, BKR15, by considering suitable minimization problems to conclude the following results.

Lemma 3.14. Let $(\mathcal{M}, \mathcal{R})=(1,1)$ or $(\mathcal{M}, \mathcal{R})=\left((-\Delta)^{\frac{1}{2}}, 1\right)$, where 1 here stands for identity operator. Then there exists a function $\Pi: s \mapsto \Pi(s), s \geq 0$, which belongs to

$$
\mathcal{P}:=\left\{\begin{array}{l|l}
P \in L^{\infty}\left(\mathbb{R}_{0}, \mathcal{L}(H)\right) & \begin{array}{l}
P(t) \text { is self-adjoint positive definite for all } t \geq 0, \\
\text { the family }\{P(t) \mid t \geq 0\} \text { is continuous in the } \\
\text { weak operator topology }
\end{array}
\end{array}\right\}
$$

and satisfies the differential Riccati equation

$$
\dot{\Pi}+\Pi \mathbb{A}^{a, b}+\mathbb{A}^{a, b^{*}} \Pi-\Pi B_{M} \mathcal{R}^{-1} B_{M}^{*} \Pi+\bar{\lambda} \Pi+\mathcal{M}^{*} \mathcal{M}=0,
$$

with $\mathbb{A}^{a, b} z:=\Delta z-a z-\nabla \cdot(b z)$. Moreover, $\Pi=\Pi_{\bar{\lambda}}$ is the unique solution of (34) in the class $\mathcal{P}$. Furthermore there is a constant $\bar{C}_{\left[C_{\mathcal{W}}, \bar{\lambda}, \frac{1}{\lambda}\right]}$ such that

$$
\left|\Pi_{\bar{\lambda}}(s)\right|_{\mathcal{L}(H)} \leq \bar{C}_{\left[C_{\mathcal{W}}, \bar{\lambda}, \frac{1}{\lambda}\right]}, \quad \text { for all } s \geq 0 .
$$

We recall that the obtained feedback control is the one that minimizes the cost function

$$
J(\eta)=J(z(\eta), \eta):=\int_{s_{0}}^{+\infty}|\mathcal{M} z(\tau)|_{H}^{2}+(\mathcal{R} \eta(\tau), \eta(\tau))_{H} \mathrm{~d} \tau
$$

Let us now consider the closed-loop system

$$
\begin{aligned}
& \partial_{t} \tilde{z}-\Delta \tilde{z}+\left(a-\frac{\bar{\lambda}}{2}\right) \tilde{z}+\nabla \cdot(b \tilde{z})+B_{M} \mathcal{R}^{-1} B_{M}^{*} \Pi_{\bar{\lambda}} \tilde{z}=0, \\
& \left.\tilde{z}\right|_{\Gamma}=0, \quad \tilde{z}\left(s_{0}\right)=z_{0} .
\end{aligned}
$$

Theorem 3.15. Let $\chi$ and $P_{M}$ satisfy the conditions in Theorem 3.12, let $(\mathcal{M}, \mathcal{R})=(1,1)$ or $(\mathcal{M}, \mathcal{R})=\left((-\Delta)^{\frac{1}{2}}, 1\right)$, and let $z_{0} \in H$. Then the solution $\tilde{z}$ for (37) is defined for all $t \geq s_{0}$, and it satisfies

$$
|\tilde{z}|_{W\left(\mathbb{R}_{s_{0}}, V, V^{\prime}\right)}^{2} \leq \bar{C}_{\left[C_{\mathcal{W}}, \bar{\lambda}, \frac{1}{\lambda}\right]}\left|z_{0}\right|_{H}^{2}
$$

Notice that that $\tilde{z}$ solves $(37)$ if, and only if, $z=\mathrm{e}^{-\left(\cdot-s_{0}\right) \frac{\bar{\lambda}}{2}} \tilde{z}(t)$ solves

$$
\begin{aligned}
& \partial_{t} z-\Delta z+a z+\nabla \cdot(b z)+B_{M} \mathcal{R}^{-1} B_{M}^{*} \Pi_{\bar{\lambda}} z=0, \\
& \left.z\right|_{\Gamma}=0, \quad z\left(s_{0}\right)=z_{0} .
\end{aligned}
$$

Therefore we can conclude the next result.

Corollary 3.16. Under the assumptions of Theorem 3.15, let $\Pi_{\bar{\lambda}} \in \mathcal{P}$ be the unique solution of (34). Then for any $z_{0} \in H$, the solution $z$ of $(39)$ is defined globally and satisfies, for all $t \geq s_{0}$,

$$
\mathrm{e}^{\left(t-s_{0}\right) \bar{\lambda}}|z(t)|_{H}^{2}+\int_{s_{0}}^{t} \mathrm{e}^{\left(\tau-s_{0}\right) \bar{\lambda}}\left(|z(\tau)|_{V}^{2}+\left|\partial_{t} z(\tau)\right|_{V^{\prime}}^{2}\right) \mathrm{d} \tau \leq \bar{C}_{\left[C_{\mathcal{W}, \mathrm{st}}, \bar{\lambda}, \frac{1}{\lambda}\right]}\left|z_{0}\right|_{H}^{2} .
$$


3.6. Dependence of the transient bound on the exponential rate. From Theorem 3.12 we have that, for a suitable open-loop control $\eta$, the solution of system (19) satisfies

$$
|z(t)|_{H}^{2} \leq C_{\bar{\lambda}} \mathrm{e}^{-\bar{\lambda}\left(t-s_{0}\right)}\left|z_{0}\right|_{H}^{2}, \text { for } t \geq s_{0}
$$

where

$$
C_{\bar{\lambda}} \leq \mathrm{e}^{\mathcal{T}_{0,2}+\mathcal{T}_{1,2} \bar{\lambda}^{2}}
$$

for suitable positive constants $\mathcal{T}_{0,2}$ and $\mathcal{T}_{1,2}$, which can be taken independent of $\bar{\lambda}$. Taking, for example, $\widehat{\lambda}=\frac{\bar{\lambda}}{2}$, again by Theorem 3.12 , we have that $J(z(\eta), \eta) \leq \widetilde{C}_{\bar{\lambda}} \mathrm{e}^{-\bar{\lambda}\left(t-s_{0}\right)}\left|z_{0}\right|_{H}^{2}$, with $\widetilde{C}_{\bar{\lambda}} \leq \mathrm{e}^{\widetilde{\mathcal{T}}_{0,2}+\widetilde{\mathcal{T}}_{1,2} \bar{\lambda}^{2}}$ and $J$ as in (36). In particular, the solution of the closed-loop system (37) also satisfies

$$
|\tilde{z}(t)|_{H}^{2} \leq \widehat{C}_{\bar{\lambda}}\left|z_{0}\right|_{H}^{2}, \quad \widehat{C}_{\bar{\lambda}} \leq \mathrm{e}^{\widehat{\mathcal{T}}_{0,2}+\widehat{\mathcal{T}}_{1,2} \bar{\lambda}^{2}}
$$

Remark 3.17. In the case $a \in L^{\infty}\left(I, L^{\infty}\right)$ we have a slightly different observability inequality (cf. [DZZ08]), and we can also prove that we can take $\Upsilon_{0}=\mathrm{e}^{\left(A_{1} \bar{\lambda}+B_{2}\right) T_{*}}$ for suitable constants $A_{1}$ and $A_{2}$, in Theorem 3.12 . In this case we would arrive to an estimate in the form $C_{\bar{\lambda}} \leq \mathrm{e}^{\mathcal{T}_{0,1}+\mathcal{T}_{1,1} \bar{\lambda}}$. Notice that in either case we have a term $\bar{\lambda}^{e}$ with $e \geq 1$.

Of course, the constants $C_{\bar{\lambda}}, \widetilde{C}_{\bar{\lambda}}$, and $\widehat{C}_{\bar{\lambda}}$ above are related to each other.

We will prove that we can have (40b) with $C_{\bar{\lambda}} \leq \mathrm{e}^{\mathcal{T}_{0, \frac{1}{2}}+\mathcal{T}_{1, \frac{1}{2}} \bar{\lambda}^{\frac{1}{2}}}$. The motivations of this study are the following:

- a sufficient condition, on the smallness of $\frac{\widehat{C}_{\bar{\lambda}}}{\bar{\lambda}}$, given in [BKR15] for the stabilizability of the FitzHugh-Nagumo system.

- we need to know the constant $\widehat{C}_{\bar{\lambda}}$ to guarantee that at time $t=\tau>s_{0}$ we will be closer to zero than we are at time $t=s_{0}$, because from $|\tilde{z}(\tau)|_{H}^{2} \leq$ $C_{\bar{\lambda}} \mathrm{e}^{-\bar{\lambda}\left(\tau-s_{0}\right)}\left|\tilde{z}\left(s_{0}\right)\right|_{H}^{2}$ it follows that

$$
|\tilde{z}(\tau)|_{H}^{2}<\left|\tilde{z}\left(s_{0}\right)\right|_{H}^{2} \quad \text { if } \quad \tau>s_{0}+\frac{\log \left(\widehat{C}_{\bar{\lambda}}\right)}{\bar{\lambda}} .
$$

- as we will see, the value $\epsilon$ in (3) will decrease as $\widehat{C}_{\bar{\lambda}}$ increases, that is the linearization based feedback control will work, for the nonlinear system, in a bigger neighborhood for a smaller $\widehat{C}_{\bar{\lambda}}$ (cf. Remark 4.10 below).

Remark 3.18. Notice also that necessarily $\widehat{C}_{\bar{\lambda}} \geq 1$. Then $\frac{\widehat{C}_{\bar{\lambda}}}{\bar{\lambda}} \rightarrow+\infty$ as $\bar{\lambda} \rightarrow 0$. It follows that, if $\widehat{C}_{\bar{\lambda}} \approx \mathrm{e}^{\widehat{\mathcal{T}}_{0, \frac{1}{2}}+\widehat{\mathcal{T}}_{1, \frac{1}{2}} \overline{\bar{\lambda}}^{\frac{1}{2}}}$ for big $\bar{\lambda}$, then the function $\frac{\widehat{C}_{\bar{\lambda}}}{\bar{\lambda}}$ has a strictly positive minimum. That is, $\frac{\widehat{C}_{\bar{\lambda}}}{\bar{\lambda}}$ cannot be made arbitrarily small by the choice of $\bar{\lambda}$.

Following [HPP01, since associated to a closed-loop system we will call the constant $\widehat{C}_{\bar{\lambda}}$ the transient bound associated to that system. The following Theorem is, however, concerned with open-loop controls and with no restriction on the dimension of the control.

Theorem 3.19. There exist $T_{* *}>0$ and nonnegative constants $\mathcal{T}_{0, \frac{1}{2}}$, and $\mathcal{T}_{1, \frac{1}{2}}$, such that for a suitable control function $\eta$, the solution of system (15), with $B=1_{\omega}$, satisfies for all $z_{0} \in H$

$$
|z(t)|_{H}^{2} \leq \mathrm{e}^{\mathcal{T}_{0, \frac{1}{2}}+\mathcal{T}_{1, \frac{1}{2}} \bar{\lambda}^{\frac{1}{2}}} \mathrm{e}^{-\bar{\lambda}\left(t-s_{0}\right)}\left|z_{0}\right|_{H}^{2}, \text { for } t \geq s_{0}
$$

and $z(t)=0$ for all $t \geq s_{0}+T_{* *}$. 
Proof. Given $T>0$ and taking $s_{1}=s_{0}+T$, by Lemma 3.11 (extending the control by zero for $t>s_{1}$ ) we have

$$
\begin{aligned}
|z(t)|_{H}^{2} \leq & \mathrm{e}^{D_{\mathrm{rc}}|(a, b)|_{\mathcal{W}^{I}}^{2} T}\left|z\left(s_{0}\right)\right|_{H}^{2} \\
& +2|\iota|_{\mathcal{L}\left(H, V^{\prime}\right)}^{2} \mathrm{e}^{\left.D_{\mathrm{rc}}|(a, b)|_{\mathcal{W}^{I}}^{2} T+\widehat{D} \Theta\left(T,|a|_{L^{\infty}\left(\mathbb{R}_{0}, L^{d}\right)}\right)|b|_{L_{\mathcal{W}}^{\infty}\left(\mathbb{R}_{0}, L^{\infty}\right)}, d\right)}\left|z\left(s_{0}\right)\right|_{H}^{2}
\end{aligned}
$$

for all $t>s_{0}$, and $z(t)=0$ for all $t \geq s_{0}+T$. Thus we can write

$$
|z(t)|_{H}^{2} \leq \mathrm{e}^{\rho_{0}\left(1+\frac{1}{T}\right)+\rho_{1}(1+\bar{\lambda}) T}\left|z\left(s_{0}\right)\right|_{H}^{2}
$$

for suitable nonnegative constants $\rho_{0}$ and $\rho_{1}$ (depending on $|(a, b)|_{\mathcal{W}}$ and $d$, but neither on $T$ nor on $\bar{\lambda}$ ).

Taking the minimizer $T_{* *}>0$ of $\mathrm{e}^{\rho_{0}\left(1+\frac{1}{*}\right)+\rho_{1}(1+\bar{\lambda})} \cdot$ is given by $\frac{\rho_{0}}{T_{* *}^{2}}=\rho_{1}(1+\bar{\lambda})$, we find

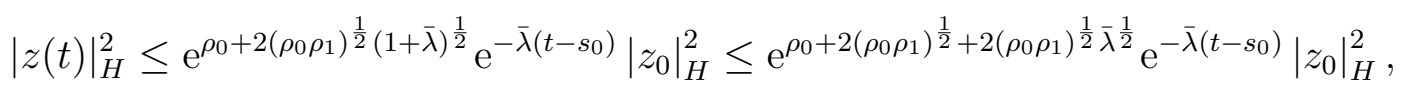

for all $t \geq s_{0}$, and $z(t)=0$ for all $t \geq s_{0}+T_{* *}$.

3.7. Remark on the viscosity coefficient. In section 2 we have rescaled time to normalize the viscosity parameter. We recall that the dimension of the stabilizing control may depend on the viscosity parameter. If we are given the system

$$
\partial_{t} z-\nu \Delta z+a z+\nabla \cdot(b z)+\sum_{i=1}^{M} u_{i} \Phi_{i}=0 ;\left.\quad z\right|_{\Gamma}=0 .
$$

after rescaling time $t=\frac{\tau}{\nu}$ we arrive to system

$$
\partial_{\tau} \breve{z}-\Delta \breve{z}+\frac{\breve{a}}{\nu} \breve{z}+\nabla \cdot\left(\frac{\breve{b}}{\nu} \breve{z}\right)+\sum_{i=1}^{M} \breve{u}_{i} \Phi_{i}=0 ;\left.\quad \breve{z}\right|_{\Gamma}=0 .
$$

with $\breve{f}(\tau):=f\left(\frac{\tau}{\nu}\right)$. Then if we want to have

$$
|z(t)|_{H}^{2} \leq C \mathrm{e}^{-\bar{\lambda} t}|z(0)|_{H}^{2}
$$

then we must have

$$
|\breve{z}(\tau)|_{H}^{2} \leq C \mathrm{e}^{-\frac{\bar{\lambda}}{\nu}}|z(0)|_{H}^{2} .
$$

From Theorem 3.12, condition (24) is sufficient for stabilization. That condition reads now

$$
\begin{aligned}
\left|1_{\omega} \chi\left(1-P_{M}\right) 1_{\omega}\right|_{\mathcal{L}\left(H, V^{\prime}\right)} & \leq 2|\iota|_{\mathcal{L}\left(H, V^{\prime}\right)}^{-2} \mathrm{e}^{-\bar{\Theta}\left(\left.\left|\frac{\breve{a}}{\nu}-\frac{\bar{\lambda}}{2 \nu}\right|_{L^{\infty}\left(I, L^{d}\right)}|| \frac{\breve{b}}{\nu}\right|_{L_{w}^{\infty}\left(I, L^{\infty}\right)}\left|\left(\frac{\breve{a}}{\nu}-\frac{\bar{\lambda}}{2 \nu}, \frac{\breve{b}}{\nu}\right)\right|_{\mathcal{W}^{I}}, d, \nu\right)} \\
& =2|\iota|_{\mathcal{L}\left(H, V^{\prime}\right)}^{-2} \mathrm{e}^{-\bar{\Theta}\left(\left|\frac{a}{\nu}-\frac{\bar{\lambda}}{2 \nu}\right|_{L^{\infty}\left(I, L^{d}\right)},\left|\frac{b}{\nu}\right|_{L_{w}^{\infty}\left(I, L^{\infty}\right)}\left|\left(\frac{a}{\nu}-\frac{\bar{\lambda}}{2 \nu}, \frac{b}{\nu}\right)\right|_{\mathcal{W}^{I}}, d, \nu\right)},
\end{aligned}
$$

with $\bar{\Theta}$ as in (23). Therefore, $P_{M}$ must be closer to the identity operator for smaller $\nu$. This is natural, because for smaller $\nu$ the system is "less stable". In other words, in order to stabilize the system, we expect to need more controls as $\nu$ decreases. Recall the estimates (26) and (27) in the examples in section 3.4. 


\section{LOCAL INTERNAL STABILIZATION OF THE NONLINEAR SYSTEM}

Here we show that the feedback rule $-B_{M} \mathcal{R}^{-1} B_{M}^{*} \Pi_{\bar{\lambda}}(t)$ constructed to stabilize exponentially the linear system $(39)$ to zero, with rate $\frac{\bar{\lambda}}{2}$, also stabilizes the nonlinear system

$$
\begin{aligned}
& \partial_{t} z-\Delta z+a z+\nabla \cdot(b z)+B_{M} \mathcal{R}^{-1} B_{M}^{*} \Pi_{\bar{\lambda}} z=\mathcal{N}(z), \\
& \left.z\right|_{\Gamma}=0, \quad z\left(s_{0}\right)=z_{0} .
\end{aligned}
$$

to zero, with the same rate, provided $z_{0}$ is small enough (cf. system(6), and provided the nonlinearity $\mathcal{N}$ satisfies suitable boundedness properties.

We follow a standard procedure by using a suitable fixed point argument. To deal with the nonlinear system we will need to ask more regularity for the reference trajectory, or more precisely to the function $b$ we obtain in the convection part of the linearized system (10a), after linearization around that reference trajectory.

This additional regularity will allow us to construct appropriate Banach spaces where we can construct an appropriate contraction mapping, whose fixed point is the solution of the nonlinear system 42 .

Implicit boundedness assumption on the the reference trajectory. Consider the spaces

$$
\begin{array}{lll}
\mathcal{W}_{\text {st }}^{J}:=\left\{b \in \mathcal{W}^{J} \mid \nabla \cdot b \in L^{\infty}\left(J, L^{2}(\Omega, \mathbb{R})\right)\right\}, & d \in\{1,2,3\}, \\
\mathcal{W}_{\mathrm{st}}:=\left\{b \in \mathcal{W} \mid \nabla \cdot b \in L^{\infty}\left(\mathbb{R}_{0}, L^{2}(\Omega, \mathbb{R})\right)\right\}, & \\
\mathcal{W}_{\mathrm{st}}^{J}:=\left\{b \in \mathcal{W}^{J} \mid \nabla \cdot b \in L_{w}^{\infty}\left(J, L^{\infty}(\Omega, \mathbb{R})\right)\right\}, & d \geq 4, \\
\mathcal{W}_{\mathrm{st}}:=\left\{b \in \mathcal{W} \mid \nabla \cdot b \in L_{w}^{\infty}\left(\mathbb{R}_{0}, L^{\infty}(\Omega, \mathbb{R})\right)\right\}, &
\end{array}
$$

(cf. (8)) which are supposed to be endowed, respectively, with the norms

$$
\begin{aligned}
|(a, b)|_{\mathcal{W}_{\mathrm{st}}^{J}} & :=\left(\left(|(a, b)|_{\mathcal{W}^{J}}^{2}+|\nabla \cdot b|_{L^{\infty}\left(J, L^{r}\right)}^{2}\right)^{\frac{1}{2}}\right. \\
|b|_{\mathcal{W}_{\mathrm{st}}} & :=\left(\left(|(a, b)|_{\mathcal{W}}^{2}+|\nabla \cdot b|_{L_{w}^{\infty}\left(\mathbb{R}_{0}, L^{r}\right)}^{2}\right)^{\frac{1}{2}} .\right.
\end{aligned}
$$

with $r=2$ for $d \in\{1,2,3\}$, and $r=\infty$ for $d \geq 4$. The spaces 43) are defined essentially for us to be able to derive the existence of strong solutions. Notice that for $1<r<+\infty$ we have $L_{w}^{\infty}\left(\mathbb{R}_{0}, L^{r}\right)=L^{\infty}\left(\mathbb{R}_{0}, L^{r}\right)$, because $L^{r}$ and $L^{r_{*}}$, with $\frac{1}{r}+\frac{1}{r_{*}}=1$, are both separable. See [Fat99, Lemma 9.1.2] and [Fat05, Section 4.1].

Now, we have the following estimates for the convection term

$$
|\nabla \cdot(b z)|_{H}=|(\nabla \cdot b) z+b \cdot \nabla z|_{H} \leq|(\nabla \cdot b)|_{L^{2}}|z|_{L^{\infty}}+|b|_{L^{\infty}}|z|_{V}
$$

and, by using the Agmon inequality (cf. (11) and [Tem97, chapter II, section 1.4]),

$$
\begin{array}{ll}
|\nabla \cdot(b z)|_{H} \leq C|(\nabla \cdot b)|_{L^{2}}|z|_{H}^{\frac{1}{2}}|z|_{V}^{\frac{1}{2}}+|b|_{L^{\infty}}|z|_{V}, & \text { for } \quad d=1 . \\
|\nabla \cdot(b z)|_{H} \leq C|(\nabla \cdot b)|_{L^{2}}|z|_{H}^{\frac{1}{2}}|z|_{\mathrm{D}(\Delta)}^{\frac{1}{2}}+|b|_{L^{\infty}}|z|_{V}, & \text { for } \quad d=2 . \\
|\nabla \cdot(b z)|_{H} \leq C|(\nabla \cdot b)|_{L^{2}}|z|_{V}^{\frac{1}{2}}|z|_{\mathrm{D}(\Delta)}^{\frac{1}{2}}+|b|_{L^{\infty}}|z|_{V}, & \text { for } \quad d=3 .
\end{array}
$$

For $d \geq 4$, the Agmon inequality does not allow us to bound the $L^{\infty}$-norm by the $\mathrm{D}(\Delta)$ norm. This is the reason we (need to) take different spaces in $43 \mathrm{~b}$ ). Notice that

$$
|\nabla \cdot(b z)|_{H} \leq C|\nabla \cdot b|_{L^{\infty}}|z|_{H}+|b|_{L^{\infty}}|z|_{V}, \quad \text { for all } \quad d \geq 1 .
$$

where $C>0$ is a positive constant.

Remark 4.1. Considering (43) we will be able to treat a wide class of nonlinearities. However, it may happen that we do not need to deal with strong solutions (see for example the case of 1D Burgers equation considered in [KR15b]), or it may happen that that we do not need to ask extra regularity for $(a, b)$ to have strong solutions (see for 
example the case the convection term takes the form $b \cdot \nabla z$ as in [BKR15], suitable to deal with Neumann boundary conditions). That is, depending on the particular system we are dealing with, we may consider different (less regular) spaces (43).

Boundedness assumption on the nonlinearity. We suppose the nonlinearity $\mathcal{N}(z)$ in system (42) satisfies for a suitable constant $\widehat{C} \geq 0$, and all $(z, \tilde{z}) \in \mathrm{D}(\Delta) \times \mathrm{D}(\Delta)$, the estimates

$$
\begin{aligned}
|\mathcal{N}(z)-\mathcal{N}(\tilde{z})|_{H}^{2} \leq & \widehat{C}|z-\tilde{z}|_{V}^{2}\left(1+|z|_{V}^{\varepsilon_{1}}+|\tilde{z}|_{V}^{\varepsilon_{2}}\right)\left(|z|_{\mathrm{D}(\Delta)}^{2}+|\tilde{z}|_{\mathrm{D}(\Delta)}^{2}\right) \\
& +\widehat{C}|z-\tilde{z}|_{\mathrm{D}(A)}^{2}\left(|z|_{V}^{\varepsilon_{3}}+|\tilde{z}|_{V}^{\varepsilon_{4}}\right),
\end{aligned}
$$

with $\left\{\varepsilon_{1}, \varepsilon_{2}\right\} \in[0,+\infty)$, and $\left\{\varepsilon_{3}, \varepsilon_{4}\right\} \in[2,+\infty)$. Notice that (44) implies that

$$
\begin{aligned}
& (\mathcal{N}(z)-\mathcal{N}(\tilde{z}), z-\tilde{z})_{H} \\
\leq & 2 \widehat{C}\left(|z|_{V}^{\varepsilon_{3}}+|\tilde{z}|_{V}^{\varepsilon_{4}}+\left(|z|_{V}^{2}+|\tilde{z}|_{V}^{2}\right)\left(1+|z|_{V}^{\varepsilon_{1}}+|\tilde{z}|_{V}^{\varepsilon_{2}}\right)\right)\left(|z|_{\mathrm{D}(\Delta)}^{2}+|\tilde{z}|_{\mathrm{D}(\Delta)}^{2}\right)|z-\tilde{z}|_{H}^{2} \\
\leq & \widehat{C}_{1}\left(1+|z|_{V}^{\varepsilon_{5}}+|\tilde{z}|_{V}^{\varepsilon_{6}}\right)\left(|z|_{\mathrm{D}(\Delta)}^{2}+|\tilde{z}|_{\mathrm{D}(\Delta)}^{2}\right)|z-\tilde{z}|_{H}^{2},
\end{aligned}
$$

for suitable $\left\{\varepsilon_{5}, \varepsilon_{6}\right\} \in[2,+\infty)$.

4.1. Strong solutions for the linearized systems. We fix $a$ and $b$, which may depend on time and space, and a constant $C_{\mathcal{W} \text {,st }} \geq 0$, satisfying

$$
|a|_{\mathcal{W}}+|b|_{\mathcal{W}_{\mathrm{st}}^{d}} \leq C_{\mathcal{W}, \mathrm{st}}
$$

We present now some results, whose proofs can be done by following the arguments in [BKR15, section 2].

By multiplying (10a) by $-2 \Delta z$, instead of by $2 z$ as in the proof of Lemma 3.3 , we can also obtain the following.

Lemma 4.2. Given $f \in L^{2}(I, H)$ and $z_{0} \in V$, there exists a strong solution $z \in$ $W(I, \mathrm{D}(A), H)$ for system (10), which depends continuously on the data:

$$
|z|_{W(I, \mathrm{D}(A), H)}^{2} \leq \bar{C}_{\left[|I|, C_{\mathcal{W}, \mathrm{st}}\right]}\left(\left|z\left(s_{0}\right)\right|_{V}^{2}+|f|_{L^{2}(I, H)}^{2}\right) .
$$

The next lemma shows a certain smoothing property of system 10 .

Lemma 4.3. Given $f \in L^{2}(I, H)$ and $z_{0} \in H$, let $z$ be the weak solution for system (10). Then $y(t):=\left(t-s_{0}\right) z(t)$ is in $W(I, \mathrm{D}(A), H)$ and satisfies the estimates

$$
\begin{aligned}
& |y|_{W(I, \mathrm{D}(A), H)}^{2} \leq \bar{C}_{\left[|I|, C_{\mathcal{W}, \mathrm{st}}\right]}\left(\left(s_{1}-s_{0}\right)^{2}|f|_{L^{2}(I, H)}^{2}+|z|_{L^{2}(I, H)}^{2}\right) \\
\leq & \bar{C}_{\left[|I|, C_{\mathcal{W}, \mathrm{st}}\right]}\left(\left|z\left(s_{0}\right)\right|_{H}^{2}+|f|_{L^{2}(I, H)}^{2}\right) .
\end{aligned}
$$

Definition 4.4. For $f \in L_{\text {loc }}^{2}\left(\mathbb{R}_{s_{0}}, H\right)$ and $y_{0} \in H$ the function $z$ defined in $\mathbb{R}_{s_{0}} \times \Omega$ by the property that $\left.z\right|_{\left(s_{0}, \tau\right)}$ coincides with the weak solution of 10$)$ in $\left(s_{0}, \tau\right)$, for all $\tau>s_{0}$ is well defined. It is called the global weak solution of 10$)$ in $\mathbb{R}_{s_{0}} \times \Omega$.

We have the following property for the solutions of 10 on the infinite time interval $\mathbb{R}_{s_{0}}=\left(s_{0},+\infty\right), s_{0} \geq 0$.

Lemma 4.5. For $f \in L^{2}\left(\mathbb{R}_{s_{0}}, V^{\prime}\right)$ and $z_{0} \in H$, let $z$ be the global weak solution of 10 , in $\mathbb{R}_{s_{0}}$, with $z\left(s_{0}\right)=z_{0}$. If $z \in L^{2}\left(\mathbb{R}_{s_{0}}, H\right)$, then $z \in W\left(\mathbb{R}_{s_{0}}, V, V^{\prime}\right)$, and

$$
|z|_{W\left(\mathbb{R}_{s_{0}}, V, V^{\prime}\right)} \leq \bar{C}_{\left[C_{\mathcal{W}, \mathrm{st}}\right]}\left(\left|z\left(s_{0}\right)\right|_{H}^{2}+|f|_{L^{2}\left(\mathbb{R}_{s_{0}}, V^{\prime}\right)}^{2}+|z|_{L^{2}\left(\mathbb{R}_{s_{0}}, H\right)}^{2}\right) .
$$


Strong solutions for the closed-loop linear system. The above Lemmas allow us to conclude the next result (cf. [BKR15, Corollary 2.19]).

Corollary 4.6. Under the assumptions of Theorem 3.15, let $\Pi \in \mathcal{P}$ be the unique solution of (34), and let $(a, b)$ satisfy (46). Then for any $z_{0} \in V$, the solution $z$ of $(39)$ is defined globally and satisfies, for all $t \geq s_{0}$,

$$
|z(t)|_{V}^{2}+|z|_{L^{2}((t, t+1), \mathrm{D}(A))}^{2} \leq \bar{C}_{\left[C_{\mathcal{W}, \mathrm{st}}, \bar{\lambda}, \frac{1}{\lambda}\right]} \mathrm{e}^{-\left(t-s_{0}\right) \bar{\lambda}}\left|z_{0}\right|_{V}^{2}
$$

4.2. Fixed point argument. We start with the following result which follows from Corollary 4.6 and Lemma 4.2 .

Corollary 4.7. Under the assumptions of Corollary 4.6, with $z_{0} \in V$, the solution $z$ of (39) is defined globally and satisfies,

$$
\sup _{t \geq s_{0}}\left|\mathrm{e}^{\bar{\lambda}\left(\cdot-s_{0}\right)} z(\cdot)\right|_{W((t, t+1), \mathrm{D}(A), H)}^{2} \leq \bar{C}_{\left[C_{\mathcal{W}, \mathrm{st}}, \bar{\lambda}, \frac{1}{\bar{\lambda}}\right]}\left|z_{0}\right|_{V}^{2}
$$

Inspired from Corollary 4.7, taking $s_{0}=0$, we define the Banach space

$$
\mathcal{Z}^{\bar{\lambda}}:=\left\{\left.z \in L_{\text {loc }}^{2}\left(\mathbb{R}_{0}, H\right)|| z\right|_{\mathcal{Z}^{\bar{\lambda}}}<\infty\right\}
$$

endowed with the norm $|z|_{\mathcal{Z}^{\bar{\lambda}}}:=\sup _{r \geq 0}\left|\mathrm{e}^{\bar{\lambda} \cdot} z\right|_{W((r, r+1), \mathrm{D}(A), H)}$. We also set

$$
\left.\mathcal{Z}_{\text {loc }}^{\bar{\lambda}}:=\left.\left\{z \in L_{\text {loc }}^{2}\left(\mathbb{R}_{0}, H\right)\right)|| \mathrm{e}^{\bar{\lambda} \cdot} z\right|_{W((r, r+1), \mathrm{D}(A), H)}<\infty \text {, for all } r \geq 0\right\} \text {. }
$$

For a given constant $\varrho>0$ and $z_{0} \in V$ we define the subset

$$
\mathcal{Z}_{\varrho}^{\bar{\lambda}}:=\left\{\left.z \in \mathcal{Z}^{\bar{\lambda}}|| z\right|_{\mathcal{Z}^{\bar{\lambda}}} ^{2} \leq \varrho\left|z_{0}\right|_{V}^{2}\right\}
$$

and the mapping $\Psi: \mathcal{Z}_{\varrho}^{\bar{\lambda}} \rightarrow \mathcal{Z}_{\text {loc }}^{\bar{\lambda}}, \bar{z} \mapsto z$, taking a given vector $\bar{z}$ to the solution $z$ of

$$
\begin{aligned}
& \partial_{t} z-\nu \Delta z+a z+\nabla \cdot(b z)+B_{M} \mathcal{R}^{-1} B_{M}^{*} \Pi_{\bar{\lambda}} z=\mathcal{N}(\bar{z}), \\
& \left.z\right|_{\Gamma}=0, \quad z\left(s_{0}\right)=z_{0} .
\end{aligned}
$$

Lemma 4.8. Under the hypotheses of Corollary 4.7, there exists $\varrho>0$ such that the following property holds: for any $\gamma \in(0,1)$ one can find a constant $\epsilon=\epsilon_{\gamma}>0$ such that, for any $z_{0}$ satisfying and $\left|z_{0}\right|_{V} \leq \epsilon$, the mapping $\Psi$ takes the set $\mathcal{Z}_{\varrho}^{\bar{\lambda}}$ into itself and satisfies the inequality

$$
\left|\Psi\left(\bar{z}_{1}\right)-\Psi\left(\bar{z}_{2}\right)\right|_{\mathcal{Z}^{\bar{\lambda}}} \leq \gamma\left|\bar{z}_{1}-\bar{z}_{2}\right|_{\mathcal{Z}^{\bar{\lambda}}} \quad \text { for all } \quad \bar{z}_{1}, \bar{z}_{2} \in \mathcal{Z}_{\varrho}^{\bar{\lambda}} .
$$

Proof. We sketch the proof into 3 main steps:

(S) Step 1: a preliminary estimate. Consider the system

$$
\begin{aligned}
& \partial_{t} z-\nu \Delta z+a z+\nabla \cdot(b z)+B_{M} \mathcal{R}^{-1} B_{M}^{*} \Pi_{\bar{\lambda}} z=f, \\
& \left.z\right|_{\Gamma}=0, \quad z(0)=z_{0} .
\end{aligned}
$$

where $f \in L_{\text {loc }}^{2}\left(\mathbb{R}_{0}, H\right)$. If $z$ is the solution of system 49 with $f=0$, by Corollary 4.7 ,

$$
\sup _{r \geq 0}\left|\mathrm{e}^{\bar{\lambda} \cdot} z(\cdot)\right|_{W((r, r+1), \mathrm{D}(A), H)}^{2} \leq \bar{C}_{\left[C_{\mathcal{W}, \mathrm{st}}, \bar{\lambda}, \frac{1}{\lambda}\right]}\left|z_{0}\right|_{V}^{2} .
$$

Proceeding as in [BRS11, KR15b, BKR15, Rod15b], it follows that for nonzero $f$ we also have

$$
\sup _{r \geq 0}\left|\mathrm{e}^{\bar{\lambda} \cdot} z(\cdot)\right|_{W((r, r+1), \mathrm{D}(A), H)}^{2} \leq \bar{C}_{\left[C_{\mathcal{W}, \mathrm{st}}, \bar{\lambda}, \frac{1}{\lambda}\right]}\left(\left|z_{0}\right|_{V}^{2}+\sup _{k \in \mathbb{N}} \int_{k}^{k+1} \mathrm{e}^{4 \bar{\lambda} s}|f(s)|_{H}^{2} \mathrm{~d} s\right) .
$$


(S) Step 2: $\Psi$ maps $\mathcal{Z}_{\varrho}^{\bar{\lambda}}$ into itself, if $\left|z_{0}\right|_{V}$ is small. We will replace $f$ by $\mathcal{N}(\bar{z})$ in (51). From (44), with $(z, \tilde{z})=(\bar{z}, 0)$, we find that

$$
\begin{aligned}
& \sup _{k \in \mathbb{N}} \int_{k}^{k+1} \mathrm{e}^{4 \bar{\lambda} s}|\mathcal{N}(\bar{z})(s)|_{H}^{2} \mathrm{~d} s \\
\leq & \sup _{k \in \mathbb{N}} \sup _{s \in[k, k+1]} \widehat{C}\left(\left|\mathrm{e}^{\bar{\lambda} s} \bar{z}(s)\right|_{V}^{2}+\left|\mathrm{e}^{\bar{\lambda} s} \bar{z}(s)\right|_{V}^{2+\varepsilon_{1}}+\left|\mathrm{e}^{\bar{\lambda} s} \bar{z}(s)\right|_{V}^{\varepsilon_{3}}\right) \int_{k}^{k+1}\left|\mathrm{e}^{\bar{\lambda} s} \bar{z}(s)\right|_{\mathrm{D}(A)}^{2} \mathrm{~d} s \\
\leq & C_{1}\left(|\bar{z}|_{\mathcal{Z}^{\bar{\lambda}}}^{4}+|\bar{z}|_{\mathcal{Z}^{\bar{\lambda}}}^{4+\varepsilon_{1}}+|\bar{z}|_{\mathcal{Z}^{\bar{\lambda}}}^{\varepsilon_{3}+2}\right),
\end{aligned}
$$

because $W((k, k+1), \mathrm{D}(\Delta), H) \hookrightarrow C([k, k+1], V)$ uniformly with respect to $k \in \mathbb{N}$. Thus, inequality (51) with $f=\mathcal{N}(\bar{z})$ and $\bar{z} \in \mathcal{Z}_{\varrho}^{\bar{\lambda}}$ gives us

$$
\begin{aligned}
& |\Psi(\bar{z})|_{\mathcal{Z}^{\bar{\lambda}}}^{2} \leq \bar{C}_{\left[C_{\mathcal{W}, \mathrm{st}}, \bar{\lambda}, \frac{1}{\lambda}\right]}\left(\left|z_{0}\right|_{V}^{2}+C_{1}\left(|\bar{z}|_{\mathcal{Z}^{\bar{\lambda}}}^{4}+|\bar{z}|_{\mathcal{Z}^{\bar{\lambda}}}^{4+\varepsilon_{1}}+|\bar{z}|_{\mathcal{Z}^{\bar{\lambda}}}^{\varepsilon_{3}+2}\right)\right) \\
\leq & C_{2}\left(1+\varrho^{2}\left|z_{0}\right|_{V}^{2}+\varrho^{\frac{4+\varepsilon_{1}}{2}}\left|z_{0}\right|_{V}^{2+\varepsilon_{1}}+\varrho^{\frac{\varepsilon_{3}+2}{2}}\left|z_{0}\right|_{V}^{\varepsilon_{3}}\right)\left|z_{0}\right|_{V}^{2}
\end{aligned}
$$

and if we set $\varrho=4 C_{2}$ and $\epsilon<\min \left\{\varrho^{-1}, \varrho^{-\frac{4+\varepsilon_{1}}{2\left(2+\varepsilon_{1}\right)}}, \varrho^{-\frac{\varepsilon_{3}+2}{2 \varepsilon_{3}}}\right\}$, then we obtain

$$
|\Psi(\bar{z})|_{\mathcal{Z}^{\bar{\lambda}}}^{2} \leq C_{2}\left(1+\varrho^{2} \epsilon^{2}+\varrho^{\frac{4+\varepsilon_{1}}{2}} \epsilon^{2+\varepsilon_{1}}+\varrho^{\frac{\varepsilon_{3}+2}{2}} \epsilon^{\varepsilon_{3}}\right) \leq 4 C_{2}\left|z_{0}\right|_{V}^{2}=\varrho\left|z_{0}\right|_{V}^{2}
$$

if $\left|z_{0}\right|_{V} \leq \epsilon$, which means that $\Psi(\bar{z}) \in \mathcal{Z}_{\varrho}^{\bar{\lambda}}$.

(s) Step 3: $\Psi$ is a contraction, if $\left|z_{0}\right|_{V}$ is smaller. It remains to prove (48). Let us take two functions $\bar{z}_{1}, \bar{z}_{2} \in \mathcal{Z}_{\varrho}^{\bar{\lambda}}$ and let $\Psi\left(\bar{z}_{1}\right)$ and $\Psi\left(\bar{z}_{2}\right)$ be the corresponding solutions for (47). Set $e=\bar{z}_{1}-\bar{z}_{2}$ and $d^{\Psi}=\Psi\left(\bar{z}_{1}\right)-\Psi\left(\bar{z}_{2}\right)$. Then $d^{\Psi}$ solves (49) with $d^{\Psi}(0)=0$ and $f=\mathcal{N}\left(\bar{z}_{1}\right)-\mathcal{N}\left(\bar{z}_{2}\right)$. Therefore, by inequality (51), we have

$$
\left|\Psi\left(\bar{z}_{1}\right)-\Psi\left(\bar{z}_{2}\right)\right|_{\mathcal{Z}^{\bar{\lambda}}}^{2} \leq \bar{C}_{\left[C_{\mathcal{W}, \text { st }}, \bar{\lambda}, \frac{1}{\lambda}\right]} \sup _{t \geq 0} \int_{t}^{t+1} \mathrm{e}^{4 \bar{\lambda} s}\left|\mathcal{N}\left(\bar{z}_{1}\right)(s)-\mathcal{N}\left(\bar{z}_{2}\right)(s)\right|_{H}^{2} \mathrm{~d} s,
$$

and from

$$
\begin{aligned}
& \mathrm{e}^{4 \bar{\lambda} s}\left|\mathcal{N}\left(\bar{z}_{1}\right)(s)-\mathcal{N}\left(\bar{z}_{2}\right)(s)\right|_{H}^{2} \\
\leq & \left|\mathrm{e}^{\bar{\lambda} s} e(s)\right|_{V}^{2}\left(1+\left|\mathrm{e}^{\bar{\lambda} s} \bar{z}_{1}(s)\right|_{V}^{\varepsilon_{1}}+\left|\mathrm{e}^{\bar{\lambda} s} \bar{z}_{2}(s)\right|_{V}^{\varepsilon_{2}}\right)\left(\left|\mathrm{e}^{\bar{\lambda} s} \bar{z}_{1}(s)\right|_{\mathrm{D}(\Delta)}^{2}+\left|\mathrm{e}^{\bar{\lambda} s} \bar{z}_{2}(s)\right|_{\mathrm{D}(\Delta)}^{2}\right) \\
& +\left|\mathrm{e}^{\bar{\lambda} s} e(s)\right|_{\mathrm{D}(A)}^{2}\left(\left|\mathrm{e}^{\bar{\lambda} s} \bar{z}_{1}(s)\right|_{V}^{\varepsilon_{3}}+\left|\mathrm{e}^{\bar{\lambda} s} \bar{z}_{2}(s)\right|_{V}^{\varepsilon_{4}}\right),
\end{aligned}
$$

it follows that

$$
\left|\Psi\left(\bar{z}_{1}\right)-\Psi\left(\bar{z}_{2}\right)\right|_{\mathcal{Z}^{\bar{\lambda}}}^{2} \leq C_{3}|e|_{\mathcal{Z}^{\bar{\lambda}}}^{2}\left(1+\left|\bar{z}_{1}\right|_{\mathcal{Z}^{\bar{\lambda}}}^{\varepsilon_{1}}+\left|\bar{z}_{2}\right|_{\mathcal{Z}^{\bar{\lambda}}}^{\varepsilon_{2}}\right)\left(\left|\bar{z}_{1}\right|_{\mathcal{Z}^{\bar{\lambda}}}^{2}+\left|\bar{z}_{2}\right|_{\mathcal{Z}^{\bar{\lambda}}}^{2}+\left|\bar{z}_{1}\right|_{\mathcal{Z}^{\bar{\lambda}}}^{\varepsilon_{3}}+\left|\bar{z}_{2}\right|_{\mathcal{Z}^{\bar{\lambda}}}^{\varepsilon^{\varepsilon}}\right),
$$

and since $\bar{z}_{1}$ and $\bar{z}_{2}$ are both in $\mathcal{Z}_{\varrho}^{\bar{\lambda}}$, we arrive to

$$
\left|d^{\Psi}\right|_{\mathcal{Z}^{\bar{\lambda}}}^{2} \leq C_{3}|e|_{\mathcal{Z}^{\bar{\lambda}}}^{2}\left(1+\varrho^{\frac{\varepsilon_{1}}{2}}\left|v_{0}\right|_{V}^{\varepsilon_{1}}+\varrho^{\frac{\varepsilon_{2}}{2}}\left|v_{0}\right|_{V}^{\varepsilon_{2}}\right)\left(2 \varrho\left|v_{0}\right|_{V}^{2}+\varrho^{\frac{\varepsilon_{3}}{2}}\left|v_{0}\right|_{V}^{\varepsilon_{3}}+\varrho^{\frac{\varepsilon_{4}}{2}}\left|v_{0}\right|_{V}^{\varepsilon_{4}}\right),
$$

Choosing $\epsilon>0$, smaller than the one in Step 2, such that

$$
\epsilon<\min \left\{\varrho^{-1}, \varrho^{-\frac{4+\varepsilon_{1}}{2\left(2+\varepsilon_{1}\right)}}, \varrho^{-\frac{\varepsilon_{3}+2}{2 \varepsilon_{3}}}, \varrho^{-\frac{1}{2}},\left(\frac{\gamma^{2}}{18 C_{3}}\right)^{\frac{1}{2}} \varrho^{-\frac{1}{2}},\left(\frac{\gamma^{2}}{9 C_{3}}\right)^{\frac{1}{\varepsilon_{3}}} \varrho^{-\frac{1}{2}},\left(\frac{\gamma^{2}}{9 C_{3}}\right)^{\frac{1}{\varepsilon_{4}}} \varrho^{-\frac{1}{2}}\right\},
$$

then we have that $\Psi$ maps $\mathcal{Z}_{\varrho}^{\bar{\lambda}}$ into itself and

$$
\left|d^{\Psi}\right|_{\mathcal{Z}^{\bar{\lambda}}}^{2} \leq C_{3}|e|_{\mathcal{Z}^{\bar{\lambda}}}^{2}\left(1+\varrho^{\frac{\varepsilon_{1}}{2}} \epsilon^{\varepsilon_{1}}+\varrho^{\frac{\varepsilon_{2}}{2}} \epsilon^{\varepsilon_{2}}\right)\left(2 \varrho \epsilon^{2}+\varrho^{\frac{\varepsilon_{3}}{2}} \epsilon^{\varepsilon_{3}}+\varrho^{\frac{\varepsilon_{4}}{2}} \epsilon^{\varepsilon_{4}}\right)<C_{3}|e|_{\mathcal{Z}^{\bar{\lambda}}}^{2} 3 \frac{3 \gamma^{2}}{9 C_{3}},
$$


provided $\left|z_{0}\right|_{V}^{2} \leq \epsilon$. That is 48 holds true: $\left|\Psi\left(\bar{z}_{1}\right)-\Psi\left(\bar{z}_{2}\right)\right|_{\mathcal{Z}^{\bar{\lambda}}}^{2}<\gamma^{2}\left|\bar{z}_{1}-\bar{z}_{2}\right|_{\mathcal{Z}^{\bar{\lambda}}}^{2}$

The proof of Lemma 4.8 is complete.

The following result says that the feedback control locally stabilizes exponentially the nonlinear system 42, is locally exponentially stable with rate $\frac{\bar{\lambda}}{2}$.

Theorem 4.9. Under the hypotheses of Corollary 4.7, there is $\epsilon>0$ with the following property: if $\left|z_{0}\right|_{V} \leq \epsilon$, then there exists a solution for the system (42), in $\mathbb{R}_{0} \times \Omega$, which belongs to $L_{\text {loc }}^{2}\left(\mathbb{R}_{0}, \mathrm{D}(A)\right) \cap C([0,+\infty), V)$, is unique, and satisfies

$$
|z(t)|_{V} \leq C \mathrm{e}^{-\bar{\lambda}\left(t-s_{0}\right)}\left|z_{0}\right|_{V}, \quad \text { for all } t \geq s_{0},
$$

for a suitable constant $C$ independent of $\left(\epsilon, z_{0}\right)$.

Proof. From Lemma 4.8 and the contraction mapping principle it follows that if $z_{0} \in V$ is sufficiently small, $\left|z_{0}\right|_{V}<\epsilon$, then there exists a unique fixed point $z=\Psi(\bar{z})=\bar{z} \in \mathcal{Z}_{\varrho}^{\epsilon}$ for $\Psi$. It follows from the definitions of $\Psi$ and $Z_{\varrho}^{\epsilon}$ that $z$ solves the system (47), with $\bar{z}=z$. We can conclude that $z$ solves (42).

Further, inequality (53) can be concluded from (52).

Finally, it remains to prove the uniqueness of the solution for (42) in the space $Z:=$ $L_{\text {loc }}^{2}\left(\mathbb{R}_{0}, \mathrm{D}(A) \times H\right) \cap C([0,+\infty), V \times H) \supset \mathcal{Z}_{\rho}^{\epsilon}$. Let $z_{1}$ and $z_{2}$ be two solutions, in $Z$, for (42). It turns out that $e=z_{1}-z_{2}$ solves 49 with $f=\mathcal{N}\left(z_{1}\right)-\mathcal{N}\left(z_{2}\right)$, Using (35) and (45), we can obtain

$$
\begin{aligned}
\left\langle B_{M} \mathcal{R}^{-1} B_{M}^{*} \Pi_{\bar{\lambda}} e, e\right\rangle_{V^{\prime}, V} & \leq \bar{C}_{\left[C_{\mathcal{W}, \mathrm{st}}, \bar{\lambda}, \frac{1}{\lambda}\right]}\left|e_{v}\right|_{H}^{2}, \\
\left\langle\mathcal{F}\left(z_{1}\right)-\mathcal{F}\left(z_{2}\right), e\right\rangle_{V^{\prime}, V} & \leq \widehat{C}_{1}\left(1+\left|z_{1}\right|_{V}^{\varepsilon_{5}}+\left|z_{2}\right|_{V}^{\varepsilon_{6}}\right)\left(\left|z_{1}\right|_{\mathrm{D}(\Delta)}^{2}+\left|z_{2}\right|_{\mathrm{D}(\Delta)}^{2}\right)|e|_{H}^{2},
\end{aligned}
$$

from which we can derive, proceeding as in the proof of Lemma $(3.3)$, that

$$
\frac{\mathrm{d}}{\mathrm{dt}}|e|_{H}^{2} \leq \bar{C}_{\left[C_{\mathcal{W}, \mathrm{st}}, \bar{\lambda}, \frac{1}{\lambda}, \frac{1}{\nu}\right]}\left(1+\left|z_{1}\right|_{V}^{\varepsilon_{5}}+\left|z_{2}\right|_{V}^{\varepsilon_{6}}\right)\left(\left|z_{1}\right|_{\mathrm{D}(\Delta)}^{2}+\left|z_{2}\right|_{\mathrm{D}(\Delta)}^{2}\right)|e|_{H}^{2}
$$

Notice that the function

$$
s \mapsto \mathcal{G}(s):=\bar{C}_{\left[C_{\mathcal{W}, \mathrm{st}}, \bar{\lambda}, \frac{1}{\lambda}, \frac{1}{\nu}\right]}\left(1+\left|z_{1}(s)\right|_{V}^{\varepsilon_{5}}+\left|z_{2}(s)\right|_{V}^{\varepsilon_{6}}\right)\left(\left|z_{1}(s)\right|_{\mathrm{D}(\Delta)}^{2}+\left|z_{2}(s)\right|_{\mathrm{D}(\Delta)}^{2}\right)
$$

is locally integrable, which allow us to write

$$
|e(t)|_{H}^{2} \leq \mathrm{e}^{\int_{0}^{t} \mathcal{G}(s) \mathrm{d} s}|e(0)|_{H}^{2}=0, \quad \text { for all } t \geq 0 .
$$

That is, the uniqueness holds true: $z_{1}-z_{2}=e=0$.

Remark 4.10. Notice that from the proof of Lemma 4.8 we see that $\varrho$ increases and $\epsilon$ decreases as the transient bound $\bar{C}_{C_{\mathcal{W}, \text { st }}, \bar{\lambda}, \frac{1}{\lambda}}$ in 50 increases.

\section{LOCAL INTERNAL STABILIZATION TO TRAJECTORIES}

As a straightforward corollary to Theorem 4.9 it follows the stabilization to trajectories for system (1).

Let us be given a solution $\hat{y}$ for the uncontrolled system (1) (with $u=0$ ) with $\hat{y}_{0}:=$ $\hat{y}(0) \in H$ and such that the vector functions in (5) are such that $\widehat{\mathcal{N}}$ satisfies (44) and (45), and $(\hat{a}, \hat{b})$ satisfies (46), for suitable nonnegative constants $\widehat{C}$ and $C_{\mathcal{W} \text {,st. Notice }}$ that, recalling the notation in section 2 , in this case $\mathcal{N}:=\frac{1}{\nu} \widehat{\widehat{\mathcal{N}}}$ also satisfies 444) and (45), and $(a, b)=\left(\frac{1}{\nu} \breve{\hat{a}}, \frac{1}{\nu} \hat{b}\right)$ also satisfies 46 , 
We consider system (1)

$$
\begin{aligned}
\partial_{t} y-\nu \Delta y+f(y, \nabla y)+B_{M} \mathcal{R}^{-1} B_{M}^{*} \widehat{\Pi}_{\lambda}(y-\hat{y}) & =0 ; \\
\left.y\right|_{\Gamma}=g ; \quad y(0) & =y_{0} .
\end{aligned}
$$

with $\widehat{\Pi}_{\lambda}$ solving

$$
\dot{\widehat{\Pi}}_{\lambda}+\widehat{\Pi}_{\lambda} \mathbb{A}_{\nu}^{\hat{a}, \hat{b}}+\mathbb{A}_{\nu}^{\hat{a}, \hat{b}^{*}} \widehat{\Pi}_{\lambda}-\widehat{\Pi}_{\lambda} B_{M} \mathcal{R}^{-1} B_{M}^{*} \widehat{\Pi}_{\lambda}+\lambda \widehat{\Pi}_{\lambda}+\mathcal{M}^{*} \mathcal{M}=0
$$

with $\mathbb{A}_{\nu}^{\hat{a}, \hat{b}} z:=\nu \Delta z-\hat{a} z-\nabla \cdot(\hat{b} z)$.

Observe that $\breve{z}(\tau):=(y-\hat{y})\left(\frac{\tau}{\nu}\right)$ solves

$$
\begin{aligned}
\partial_{\tau} \breve{z}-\Delta \breve{z}+a \breve{z}+\nabla \cdot(b \breve{z})+\frac{1}{\nu} B_{M} \mathcal{R}^{-1} B_{M}^{*} \breve{\widehat{\Pi}}_{\lambda} \breve{z}=\mathcal{N}(\breve{z}) ; \\
\left.\breve{z}\right|_{\Gamma}=0 ; \quad \breve{z}(0)=y_{0}-\hat{y}_{0},
\end{aligned}
$$

and $\breve{\Pi}_{\lambda}(\tau)=\widehat{\Pi}_{\lambda}\left(\frac{\tau}{\nu}\right)$ solves 34 with a different pair $(\mathcal{R}, \mathcal{M})$ :

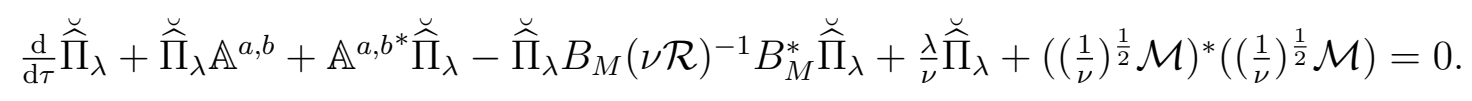

Therefore, from Theorem 4.9 , we have that

$$
|\breve{z}(\tau)|_{V}^{2} \leq C \mathrm{e}^{-\frac{\lambda}{\nu} \tau}|\breve{z}(0)|_{V}^{2}, \quad \text { for all } \tau \geq 0,
$$

provided $|\breve{z}(0)|_{V}$ is small enough. This implies that

$$
|y(t)-\hat{y}(t)|_{V}^{2} \leq C \mathrm{e}^{-\lambda t}\left|y_{0}-\hat{y}_{0}\right|_{V}^{2}, \quad \text { for all } t \geq 0,
$$

and we can conclude that the following theorem holds true.

Theorem 5.1. Under the hypotheses of Theorem 4.9, there exists $\epsilon>0$ with the following properties. If $y_{0} \in H$ is such that

$$
y_{0}-\hat{y}_{0} \in V \quad \text { and } \quad\left|y_{0}-\hat{y}_{0}\right|_{V}<\epsilon
$$

then the solution $y$ of the system (54) goes exponentially to $\hat{y}$ with rate $\frac{\lambda}{2}$, that is,

$$
|y(t)-\hat{y}(t)|_{V}^{2} \leq C \mathrm{e}^{-\lambda t}\left|y_{0}-\hat{y}_{0}\right|_{V}^{2}, \quad \text { for all } t \geq 0,
$$

for a suitable constant $C$ independent of $\left(\epsilon, y_{0}-\hat{y}_{0}\right)$. Furthermore, the solution $y$ is, and is unique, in the affine space $\hat{y}+L_{\text {loc }}^{2}\left(\mathbb{R}_{0}, \mathrm{D}(A)\right) \cap C([0,+\infty), V)$.

Notice that the feedback control stabilizes the linearized system to zero globally, that is, we have the following theorem.

Theorem 5.2. Under the hypotheses of Theorem 4.9, given $z_{0} \in V$, the solution of

$$
\begin{aligned}
\partial_{t} z-\nu \Delta z+\hat{a} z+\nabla \cdot(\hat{b} z)+B_{M} \mathcal{R}^{-1} B_{M}^{*} \widehat{\Pi}_{\lambda} z & =0 ; \\
\left.z\right|_{\Gamma}=0 ; \quad z(0) & =z_{0},
\end{aligned}
$$

satisfies

$$
|z(t)|_{V}^{2} \leq C \mathrm{e}^{-\lambda t}\left|z_{0}\right|_{V}^{2}, \quad \text { for all } t \geq 0
$$

for a suitable constant $C$ independent of $z_{0}$. Furthermore, the solution $z$ is, and is unique, in the space $L_{\text {loc }}^{2}\left(\mathbb{R}_{0}, \mathrm{D}(A)\right) \cap C([0,+\infty), V)$. 


\section{Example. Polynomial nOnlinearities}

Many systems modelling real evolutions involve polynomial nonlinearities, for example the Fisher-like equations [Fis37, VP09] modelling population dynamics and the Burgerslike equations [KR15b, BRT15] modelling fluid (e.g., traffic) flow. Here we check the previous assumptions for the case the function $f(y, \nabla y)$ takes the form $f_{\mathrm{r}}(y)+f_{\mathrm{c}}(y) \cdot \nabla y$ where $f_{\mathrm{r}}$ and $f_{\mathrm{c}}=\left[\begin{array}{llll}f_{\mathrm{c} 1} & f_{\mathrm{c} 2} & \cdots & f_{\mathrm{c} d}\end{array}\right]^{\top}$, are polynomials:

$$
f_{\mathrm{r}}(y)=\sum_{j=0}^{\bar{p}} \bar{r}_{j} y^{j} \quad \text { and } \quad f_{\mathrm{c} k}(y)=\sum_{j=0}^{p_{k}} r_{k, j} y^{j},
$$

with $\bar{r}_{j}$ and $r_{k, j}$ real numbers, and $p_{k} \in \mathbb{N}$ for $k \in\{1, \ldots, d\}$.

For illustration, we consider here the case $d=3$. The following estimates will be also valid for $d \in\{1,2\}$, though in those cases better estimates may hold true. On the other hand some of the following arguments will not work in dimension $d \geq 4$, in that case some changes are needed.

It is enough to analyze the case of monomials, with degree greater than or equal to 2 :

$$
f(y)=y^{\bar{n}} \text { with } \bar{n} \geq 2
$$

and

$$
f(y)=y^{n} \partial_{x_{\bar{k}}} y=\frac{1}{n+1} \partial_{x_{\bar{k}}} y^{n+1}, \quad \text { with } n \geq 1 \text { for some } \bar{k} \in\{1, \ldots, d\} .
$$

In this case, recalling the notation in Section 2 , for a given trajectory $\hat{y}$, we obtain respectively either

$$
\hat{a}=\bar{n} \hat{y}^{\bar{n}-1} \quad \text { and } \quad \hat{b}=0,
$$

or

$$
\hat{a}=0 \quad \text { and } \quad \hat{b}=\left[\begin{array}{llll}
\hat{b}_{1} & \hat{b}_{2} & \ldots & \hat{b}_{d}
\end{array}\right]^{\top}, \quad \text { with } \quad \hat{b}_{k}= \begin{cases}0 & \text { if } k \neq \bar{k} \\
\hat{y}^{n} & \text { if } k=\bar{k}\end{cases}
$$

Observe that in the case of a reaction nonlinearity $f(y)=y^{\bar{n}}$, condition $(9)$ is satisfied provided $\hat{y} \in L^{\infty}\left(\mathbb{R}_{0}, L^{3(\bar{n}-1)}\right)$. In the case of a convection nonlinearity $f(y)=\partial_{x_{\bar{k}}} y^{n+1}$, conditions (9) and (46) are satisfied provided $\hat{y} \in L_{w}^{\infty}\left(\mathbb{R}_{0}, L^{\infty}\right) \cap L^{\infty}\left(\mathbb{R}_{0}, W^{1,3}(\Omega, \mathbb{R})\right)$.

The nonlinearity. Concerning (44), we need to be more careful, and need a bit more of work. Again we consider only monomials. We also consider the case $\hat{y} \in L_{w}^{\infty}\left(\mathbb{R}_{0}, L^{\infty}\right)$.

Example 6.1. In the case $\mathcal{N}(z)=\hat{y}^{m} z^{2}, m \in \mathbb{N}$, (44) holds true. We may write

$$
|\mathcal{N}(z)-\mathcal{N}(\tilde{z})|_{H}^{2}=\left|\hat{y}^{m}(z-\tilde{z})(z+\tilde{z})\right|_{H}^{2} \leq|z-\tilde{z}|_{H}^{2}\left|\hat{y}^{m}\right|_{L^{\infty}}^{2}|z+\tilde{z}|_{L^{\infty}}^{2}
$$

and

$$
|\mathcal{N}(z)-\mathcal{N}(\tilde{z})|_{H}^{2} \leq C\left|\hat{y}^{m}\right|_{L^{\infty}}^{2}|z-\tilde{z}|_{V}^{2}\left(|z|_{\mathrm{D}(\Delta)}^{2}+|\tilde{z}|_{\mathrm{D}(\Delta)}^{2}\right),
$$

which shows that (44) holds true.

Example 6.2. In the case $\mathcal{N}(z)=\hat{y}^{m} z^{n}, m \in \mathbb{N}$ and $n=\{3,4,5\}$, 44 holds true. We may write, for suitable nonzero constants $r_{j}$,

$$
\mathcal{N}(z)-\mathcal{N}(\tilde{z})=\hat{y}^{m}(z-\tilde{z}) \sum_{j=0}^{n-1} r_{j} z^{j} \tilde{z}^{n-1-j}
$$


where in the sum we have monomials of degree $n-1$. For example for $z^{1} \tilde{z}^{n-2}$, by standard (yet appropriate) Young, Hölder, Sobolev, and Agmon inequalities, we may write

$$
\begin{aligned}
\left|(z-\tilde{z}) z^{1} \tilde{z}^{n-2}\right|_{H}^{2} & =\left|(z-\tilde{z})^{2} z^{2} \tilde{z}^{2 n-4}\right|_{L^{1}} \leq|z-\tilde{z}|_{L^{\infty}}^{2}|z|_{L^{\infty}}|\tilde{z}|_{L^{\infty}}\left|z \tilde{z}^{2 n-5}\right|_{L^{1}} \\
& \leq C_{1}|z-\tilde{z}|_{V}|z-\tilde{z}|_{\mathrm{D}(\Delta)}|z|_{V}^{\frac{1}{2}}|z|_{\mathrm{D}(\Delta)}^{\frac{1}{2}}|\tilde{z}|_{V}^{\frac{1}{2}}|\tilde{z}|_{\mathrm{D}(\Delta)}^{\frac{1}{2}}|z|_{L^{6}}|\tilde{z}|_{L^{\frac{6(2 n-5)}{5}}}^{2 n-5}
\end{aligned}
$$

and, since $H^{1}(\Omega, \mathbb{R}) \hookrightarrow L^{6}(\Omega, \mathbb{R}) \hookrightarrow L^{\frac{6(2 n-5)}{5}}(\Omega, \mathbb{R})$,

$$
\begin{aligned}
\left|(z-\tilde{z}) z^{1} \tilde{z}^{n-2}\right|_{H}^{2} & \leq \frac{C_{1}}{2}|z-\tilde{z}|_{V}^{2}|z|_{\mathrm{D}(\Delta)}|\tilde{z}|_{\mathrm{D}(\Delta)}+C_{2}|z-\tilde{z}|_{\mathrm{D}(\Delta)}^{2}|z|_{V}^{3}|\tilde{z}|_{V}^{1+2(2 n-5)} \\
& \leq \frac{C_{1}}{4}|z-\tilde{z}|_{V}^{2}\left(|z|_{\mathrm{D}(\Delta)}^{2}+|\tilde{z}|_{\mathrm{D}(\Delta)}^{2}\right)+C_{3}|z-\tilde{z}|_{\mathrm{D}(\Delta)}^{2}\left(|z|_{V}^{4 n-6}+|\tilde{z}|_{V}^{4 n-6}\right)
\end{aligned}
$$

For the other monomials we can obtain analogous estimates, which give us

$$
\begin{aligned}
|\mathcal{N}(z)-\mathcal{N}(\tilde{z})|_{H}^{2} \leq & C_{4}\left|\hat{y}^{m}\right|_{L^{\infty}}^{2}|z-\tilde{z}|_{V}^{2}\left(|z|_{\mathrm{D}(\Delta)}^{2}+|\tilde{z}|_{\mathrm{D}(\Delta)}^{2}\right) \\
& +C_{4}\left|\hat{y}^{m}\right|_{L^{\infty}}^{2}|z-\tilde{z}|_{\mathrm{D}(\Delta)}^{2}\left(|z|_{V}^{4 n-6}+|\tilde{z}|_{V}^{4 n-6}\right)
\end{aligned}
$$

which shows that (44) holds true.

Example 6.3. In the case $\mathcal{N}(z)=\hat{y}^{m} z^{6}$, we were not able to derive (44). Proceeding as above, for suitable nonzero constants $r_{j}$,

$$
\mathcal{N}(z)-\mathcal{N}(\tilde{z})=\hat{y}^{m}(z-\tilde{z}) \sum_{j=0}^{4} r_{j} z^{j} \tilde{z}^{5-j}
$$

where in the sum we have now monomials of degree 5 . If for example for $z^{1} \tilde{z}^{4}$, we proceed as above and write

$$
\begin{aligned}
\left|(z-\tilde{z}) z^{1} \tilde{z}^{4}\right|_{H}^{2} & =\left|(z-\tilde{z})^{2} z^{2} \tilde{z}^{8}\right|_{L^{1}} \leq|z-\tilde{z}|_{L^{\infty}}^{2}|z|_{L^{\infty}}|\tilde{z}|_{L^{\infty}}\left|z \tilde{z}^{7}\right|_{L^{1}} \\
& \leq C_{1}|z-\tilde{z}|_{V}|z-\tilde{z}|_{\mathrm{D}(\Delta)}|z|_{V}^{\frac{1}{2}}|z|_{\mathrm{D}(\Delta)}^{\frac{1}{2}}|\tilde{z}|_{V}^{\frac{1}{2}}|\tilde{z}|_{\mathrm{D}(\Delta)}^{\frac{1}{2}}\left|z \tilde{z}^{7}\right|_{L^{1}}
\end{aligned}
$$

we cannot bound the term $\left|z \tilde{z}^{7}\right|_{L^{1}}$ by the $V$-norms of $z$ and $\tilde{z}$ (for $d=3$ ). Trying to use again the $\mathrm{D}(\Delta)$-norms, we were not able to arrive to 44 (the $\mathrm{D}(\Delta)$-norms will appear with a power strictly greater than 2 ).

Example 6.4. In the case $\mathcal{N}(z)=\nabla \cdot\left(g(\hat{y}) z^{n}\right)$, where $n \in\{2,3\}$ and $g: \mathbb{R} \rightarrow \mathbb{R}^{3}$ is a smooth function, estimate (44) holds true provided $g(\hat{y}) \in \mathcal{W}_{\text {st }}$. We consider only the case $n=3$. We write, for suitable nonzero constants $r_{j}$,

$$
\mathcal{N}(z)-\mathcal{N}(\tilde{z})=\nabla \cdot\left(g(\hat{y})(z-\tilde{z}) \sum_{j=0}^{2} r_{j} z^{j} \tilde{z}^{2-j}\right)
$$


where in the sum we have monomials of degree 2. For example for $z \tilde{z}$ we find

$$
\begin{aligned}
& |\nabla \cdot(g(\hat{y})(z-\tilde{z}) z \tilde{z})|_{H}^{2} \\
\leq & \left|(\nabla \cdot g(\hat{y}))^{2}(z-\tilde{z})^{2} z^{2} \tilde{z}^{2}\right|_{L^{1}}+|g(\hat{y})|_{L^{\infty}}^{2}\left|(\nabla((z-\tilde{z}) z \tilde{z}))^{2}\right|_{L^{1}} \\
\leq & |(\nabla \cdot g(\hat{y}))|_{L^{3}}^{2}|z-\tilde{z}|_{L^{6}}^{2}\left|z^{2} \tilde{z}^{2}\right|_{L^{\infty}} \\
& +|g(\hat{y})|_{L^{\infty}}^{2}\left(\left|(\nabla(z-\tilde{z}))^{2}\right|_{L^{1}}\left|z^{2} \tilde{z}^{2}\right|_{L^{\infty}}+|z-\tilde{z}|_{L^{\infty}}^{2}\left|(\nabla(z \tilde{z}))^{2}\right|_{L^{1}}\right) \\
\leq & C|z-\tilde{z}|_{V}^{2}|z|_{L^{\infty}}^{2}|\tilde{z}|_{L^{\infty}}^{2}+C|z-\tilde{z}|_{V}|z-\tilde{z}|_{\mathrm{D}(\Delta)}\left(|z|_{V}^{2}|\tilde{z}|_{L^{\infty}}^{2}+|z|_{L^{\infty}}^{2}|\tilde{z}|_{V}^{2}\right) \\
\leq & C_{1}|z-\tilde{z}|_{V}^{2}\left(|z|_{V}^{2}+|z|_{V}^{2}\right)\left(|z|_{\mathrm{D}(\Delta)}^{2}+|\tilde{z}|_{\mathrm{D}(\Delta)}^{2}\right) \\
& +C_{1}|z-\tilde{z}|_{V}|z-\tilde{z}|_{\mathrm{D}(\Delta)}\left(|z|_{V}^{2}|\tilde{z}|_{V}|\tilde{z}|_{\mathrm{D}(\Delta)}+|z|_{V}|z|_{\mathrm{D}(\Delta)}|\tilde{z}|_{V}^{2}\right) \\
\leq & C_{2}|z-\tilde{z}|_{V}^{2}\left(|z|_{V}^{2}+|z|_{V}^{2}+1\right)\left(|z|_{\mathrm{D}(\Delta)}^{2}+|\tilde{z}|_{\mathrm{D}(\Delta)}^{2}\right)+C_{2}|z-\tilde{z}|_{\mathrm{D}(\Delta)}^{2}\left(|z|_{V}^{6}+|\tilde{z}|_{V}^{6}\right) .
\end{aligned}
$$

We can obtain analogous estimates for the other monomials, and conclude that

$$
\begin{aligned}
|\mathcal{N}(z)-\mathcal{N}(\tilde{z})|_{H}^{2} \leq & C_{3}|z-\tilde{z}|_{V}^{2}\left(|z|_{V}^{2}+|z|_{V}^{2}+1\right)\left(|z|_{\mathrm{D}(\Delta)}^{2}+|\tilde{z}|_{\mathrm{D}(\Delta)}^{2}\right) \\
& +C_{3}|z-\tilde{z}|_{\mathrm{D}(\Delta)}^{2}\left(|z|_{V}^{6}+|\tilde{z}|_{V}^{6}\right) .
\end{aligned}
$$

which shows that (44) holds true.

\section{BOUNDARY STABILIZATION}

We start by considering a linear system in the form (7), without the nonlinearity, which we rewrite in the more general form

$$
\begin{aligned}
\partial_{t} z-\Delta z+a z+\nabla \cdot(b z) & =0 ;\left.\quad z\right|_{\Gamma}=B_{\Gamma} \zeta ; \\
z(0) & =z_{0} .
\end{aligned}
$$

where now our control is a function $\zeta \in \mathcal{Z}$, where $\mathcal{Z}$ is an Hilbert space, and $B_{\Gamma} \in$ $\mathcal{L}\left(\mathcal{Z}, G_{\text {loc }}^{1}\left(\mathbb{R}_{0}, \Gamma\right)\right)$ with

$$
\begin{aligned}
G_{\mathrm{loc}}^{1}\left(\mathbb{R}_{0}, \Gamma\right) & :=\bigcap_{T>0} G^{1}((0, T), \Gamma), \\
G^{1}\left(\left(s_{0}, s_{1}\right), \Gamma\right) & :=\left.W\left(\left(s_{0}, s_{1}\right), H^{1}(\Omega, \mathbb{R}), V^{\prime}\right)\right|_{\Gamma}:=\left\{\left.v\right|_{\Gamma} \mid v \in W\left(\left(s_{0}, s_{1}\right), H^{1}(\Omega, \mathbb{R}), V^{\prime}\right)\right\},
\end{aligned}
$$

for all $s_{1}>s_{0} \geq 0$.

7.1. Weak solutions. Let us consider the more general system

$$
\begin{aligned}
& \partial_{t} z-\Delta z+a z+\nabla \cdot(b z)+g=0, \\
& \left.z\right|_{\Gamma}=\gamma, \quad z(0)=z_{0} .
\end{aligned}
$$

with an external body forcing $g$ and where the control in (57) is replaced by a general external boundary forcing $\gamma$.

Notice that for $I=\left(s_{0}, s_{1}\right)$ and for given $v \in W\left(I, H^{1}(\Omega, \mathbb{R}), V^{\prime}\right)$, we have $\left.v\right|_{\Gamma}=0$ if, and only if, $v \in W\left(I, V, V^{\prime}\right)$. Therefore since $v \in W\left(I, V, V^{\prime}\right)$ is a closed subspace of $W\left(I, H^{1}(\Omega, \mathbb{R}), V^{\prime}\right)$ we have that the function

$$
\begin{aligned}
E_{1}: G^{1}(I, \Gamma) & \rightarrow W\left(I, H^{1}(\Omega, \mathbb{R}), V^{\prime}\right) ; \\
\gamma & \mapsto E_{1} \gamma \in W\left(I, V, V^{\prime}\right)^{\perp}, \quad \text { with }\left.\quad E_{1}(\gamma)\right|_{\Gamma}=\gamma
\end{aligned}
$$


is well defined. Notice also that in this case we can see that the trace space $G^{1}(I, \Gamma)$ is an Hilbert space, with the scalar product

$$
(\gamma, \xi)_{G^{1}(I, \Gamma)}:=\left(E_{1}(\gamma), E_{1}(\xi)\right)_{W\left(I, H^{1}(\Omega, \mathbb{R}), V^{\prime}\right)} .
$$

The corresponding induced norm corresponds to the trace norm (or range norm, cf. [Rod14, Lemma 3.1])

$$
|\gamma|_{G^{1}(I, \Gamma)}:=\inf _{\gamma=\left.v\right|_{\Gamma}}|v|_{\left.W(I), H^{1}(\Omega, \mathbb{R}), V^{\prime}\right)} .
$$

Definition 7.1. We say that $z$ is a weak solution for system (58) if $y=z-E_{1} \gamma$ is a weak solution for the system (10) with $f=g+\partial_{t} E_{1} \gamma-\Delta E_{1} \gamma+a E_{1} \gamma+\nabla \cdot\left(b E_{1} \gamma\right)$, and $y(0)=z_{0}-E_{1} \gamma(0)$.

Lemma 7.2. Given $(a, b) \in \mathcal{W}$ satisfying (9), $g \in L^{2}\left(I, V^{\prime}\right), \gamma \in G^{1}(I, \Gamma)$ and $z_{0} \in H$, there exists a weak solution $z \in W\left(I, H^{1}(\Omega, \mathbb{R}), V^{\prime}\right)$ for $(10)$. Moreover $z$ is unique and depends continuously on the data:

$$
|z|_{W\left(I, H^{1}(\Omega, \mathbb{R}), V^{\prime}\right)}^{2} \leq \bar{C}_{\left[|I|, C_{\mathcal{W}}\right]}\left(\left|z\left(s_{0}\right)\right|_{H}^{2}+|g|_{L^{2}\left(I, V^{\prime}\right)}^{2}+|\gamma|_{G^{1}(I, \Gamma)}^{2}\right) .
$$

Proof. The proof is straightforward from Definition 7.1] and Lemma 3.3 (cf. [Rod14, Theorem 3.2]).

7.2. Strong solutions. In order to define strong solutions, we introduce

$$
\begin{aligned}
G_{\mathrm{loc}}^{2}\left(\mathbb{R}_{0}, \Gamma\right) & :=\bigcap_{T>0} G^{1}((0, T), \Gamma), \\
G^{2}\left(\left(s_{0}, s_{1}\right), \Gamma\right) & :=\left.W\left(\left(s_{0}, s_{1}\right), H^{2}(\Omega, \mathbb{R}), H\right)\right|_{\Gamma},
\end{aligned}
$$

for all $s_{1}>s_{0} \geq 0$, and consider the extension

$$
\begin{aligned}
E_{2}: G^{2}\left(\left(s_{0}, s_{1}\right), \Gamma\right) & \rightarrow W\left(\left(s_{0}, s_{1}\right), H^{2}(\Omega, \mathbb{R}), H\right) ; \\
\gamma & \mapsto E_{2} \gamma \in W\left(\left(s_{0}, s_{1}\right), \mathrm{D}(\Delta), H\right)^{\perp}, \quad \text { with }\left.\quad E_{2}(\gamma)\right|_{\Gamma}=\gamma
\end{aligned}
$$

The trace space $G^{2}\left(\left(s_{0}, s_{1}\right), \Gamma\right)$ is endowed with the scalar product

$$
(\gamma, \xi)_{G^{2}\left(\left(s_{0}, s_{1}\right), \Gamma\right)}:=\left(E_{2}(\gamma), E_{2}(\xi)\right)_{W\left(\left(s_{0}, s_{1}\right), H^{2}(\Omega, \mathbb{R}), H\right)}
$$

and induced norm

$$
|\gamma|_{G^{2}\left(\left(s_{0}, s_{1}\right), \Gamma\right)}:=\inf _{\gamma=\left.v\right|_{\Gamma}}|v|_{W\left(\left(s_{0}, s_{1}\right), H^{2}(\Omega, \mathbb{R}), H\right)} .
$$

Definition 7.3. We say that $z$ is a strong solution for system (58) if $y=z-E_{2} \gamma$ is a strong solution for the system (10) with $f=g+\partial_{t} E_{2} \gamma-\Delta E_{2} \gamma+a E_{2} \gamma+\nabla \cdot\left(b E_{2} \gamma\right)$, and $y(0)=z_{0}-E_{2} \gamma(0) \in V$.

Lemma 7.4. Given $(a, b) \in \mathcal{W}_{\text {st }}$ satisfying (46), $g \in L^{2}(I, H), \gamma \in G^{2}(I, \Gamma)$ and $z_{0} \in$ $H^{1}(\Omega, \mathbb{R})$, with $z_{0}-E_{2} \gamma(0) \in V$, then there exists a strong solution $z \in W\left(I, H^{2}(\Omega, \mathbb{R}), H\right)$ for (58). Moreover $z$ is unique and depends continuously on the data

$$
|z|_{W\left(I, H^{2}(\Omega, \mathbb{R}), H\right)}^{2} \leq \bar{C}_{\left[|I|, C_{\mathcal{W}, \text { st }}\right]}\left(\left|z\left(s_{0}\right)\right|_{H}^{2}+|g|_{L^{2}(I, H)}^{2}+|\gamma|_{G^{2}(I, \Gamma)}^{2}\right) .
$$

Proof. The proof is straightforward from Definition 7.3 and Lemma 4.2 .

Also in the case of nonhomogeneous boundary conditions, we have the following smoothing property. 
Lemma 7.5. Let us be given $(a, b) \in \mathcal{W}_{\text {st }}$ satisfying (46), $z_{0} \in H, g \in L^{2}(I, H)$, and $\left.\gamma \in G^{2}(I, \Gamma)\right)$; then for the weak solution $z$ of system $(58)$, we have that $\left(\cdot-s_{0}\right) z \in$ $W\left(I, H^{2}(\Omega, \mathbb{R}), H\right)$, and

$$
\left(\left|\left(\cdot-s_{0}\right) z\right|_{\left.W\left(I, H^{2}(\Omega, \mathbb{R}), H\right)\right)}^{2} \leq \bar{C}_{\left[C_{\mathcal{W}, \mathrm{st}}\right]}\left(\left|z_{0}\right|_{H}^{2}+|g|_{L^{2}(I, H)}^{2}+|\gamma|_{G^{2}(I, \Gamma)}^{2}\right) .\right.
$$

Proof. Since $z$ solves (58), it turns out that also $w=\left(\cdot-s_{0}\right) z$ does, with different data:

$$
\begin{aligned}
& \partial_{t} w-\Delta w+a w+\nabla \cdot(b w)+\left(\cdot-s_{0}\right) g-z=0, \\
& \left.w\right|_{\Gamma}=\left(\cdot-s_{0}\right) \gamma, \quad w(0)=0 .
\end{aligned}
$$

Then, from Lemma 7.4, we can derive that

$$
|w|_{W\left(I, H^{2}(\Omega, \mathbb{R}), H\right)}^{2} \leq \bar{C}_{\left[|I|, C_{\mathcal{W}, \mathrm{st}}\right]}\left(\left|\left(\cdot-s_{0}\right) g\right|_{L^{2}(I, H)}^{2}+|z|_{L^{2}(I, H)}^{2}+\left|\left(\cdot-s_{0}\right) \gamma\right|_{G^{2}(I, \Gamma)}^{2}\right) .
$$

Thus, the result follows from $|z|_{L^{2}(I, H)}^{2} \leq|z|_{W\left(I, H^{1}(\Omega, \mathbb{R}), H^{-1}(\Omega, \mathbb{R})\right)}^{2}$ and from Lemma 3.3

Lemma 7.6. Let $(a, b) \in \mathcal{W}_{\text {st }}$ satisfy (46). Let $z$ solve system (58), with $g \in L^{2}\left(\mathbb{R}_{0}, H\right)$ and $\gamma \in G^{2}\left(\mathbb{R}_{0}, \Gamma\right)$. If $z \in L^{2}\left(\mathbb{R}_{0}, H\right)$, then $z \in W\left(\mathbb{R}_{0}, H^{1}(\Omega, \mathbb{R}), H^{-1}(\Omega, \mathbb{R})\right)$, with

$$
|z|_{W\left(\mathbb{R}_{0}, H^{1}(\Omega, \mathbb{R}), H^{-1}(\Omega, \mathbb{R})\right)}^{2} \leq \bar{C}_{\left[C_{\mathcal{W}, \mathrm{st}}\right]}\left(\left|z_{0}\right|_{H}^{2}+|z|_{L^{2}\left(\mathbb{R}_{0}, H\right)}^{2}+|g|_{L^{2}\left(\mathbb{R}_{0}, H\right)}^{2}+|\gamma|_{G^{2}\left(\mathbb{R}_{0}, \Gamma\right)}^{2}\right) .
$$

Proof. From Theorem 3.3 we find

$$
|z|_{W\left((0,1), H^{1}(\Omega, \mathbb{R}), H^{-1}(\Omega, \mathbb{R})\right)}^{2} \leq \bar{C}_{\left[C_{\mathcal{W}, \mathrm{st}}\right]}\left(\left|z_{0}\right|_{H}^{2}+|g|_{L^{2}\left((0,1), V^{\prime}\right)}^{2}+|\gamma|_{G^{1}((0,1), \Gamma)}^{2}\right)
$$

and, from Lemma 7.5 we have that for all $t \geq 1$

$$
|z(t)|_{H^{1}(\Omega, \mathbb{R})}^{2} \leq \bar{C}_{\left[C_{\mathcal{W}, \mathrm{st}}\right]}\left(|v(t-1)|_{H}^{2}+|g|_{L^{2}((t-1, t), H)}^{2}+|\gamma|_{G^{2}((t-1, t), \Gamma)}^{2}\right),
$$

which allow us to obtain

$$
\begin{aligned}
& |z|_{L^{2}\left(\mathbb{R}_{1}, H^{1}(\Omega, \mathbb{R})\right)}^{2}=\sum_{n=1}^{+\infty}|z|_{L^{2}\left((n, n+1), H^{1}(\Omega, \mathbb{R})\right)}^{2} \\
\leq & \bar{C}_{\left[C_{\mathcal{W}, \mathrm{st}}\right]} \sum_{n=1}^{+\infty} \int_{n}^{n+1}|v(t-1)|_{H}^{2}+|g|_{L^{2}((t-1, t), H)}^{2}+|\gamma|_{G^{2}((t-1, t), \Gamma)}^{2} \mathrm{~d} t \\
\leq & \bar{C}_{\left[C_{\mathcal{W}, \mathrm{st}}\right]}\left(|z|_{L^{2}\left(\mathbb{R}_{0}, H\right)}^{2}+\sum_{n=1}^{+\infty} \int_{n}^{n+1}|g|_{L^{2}((t-1, t), H)}^{2}+\left|E_{2} \gamma\right|_{W^{1}\left((t-1, t), H^{2}(\Omega, \mathbb{R}), H\right)}^{2} \mathrm{~d} t\right) \\
\leq & \bar{C}_{\left[C_{\mathcal{W}, \mathrm{st}}\right]}\left(|z|_{L^{2}\left(\mathbb{R}_{0}, H\right)}^{2}+\sum_{n=1}^{+\infty}|g|_{L^{2}((n-1, n+1), H)}^{2}+\left|E_{2} \gamma\right|_{W^{1}\left((n-1, n+1), H^{2}(\Omega, \mathbb{R}), H\right)}^{2}\right) .
\end{aligned}
$$

Hence, it follows

$$
\begin{aligned}
|z|_{L^{2}\left(\mathbb{R}_{1}, H^{1}(\Omega, \mathbb{R})\right)}^{2} & \leq \bar{C}_{\left[C_{\mathcal{W}, \mathrm{st}}\right]}\left(|z|_{L^{2}\left(\mathbb{R}_{0}, H\right)}^{2}+2|g|_{L^{2}\left(\mathbb{R}_{0}, H\right)}^{2}+2\left|E_{2} \gamma\right|_{W^{1}\left(\mathbb{R}_{0}, H^{2}(\Omega, \mathbb{R}), H\right)}^{2}\right) \\
& \leq 2 \bar{C}_{\left[C_{\mathcal{W}, \mathrm{st}}\right]}\left(|z|_{L^{2}\left(\mathbb{R}_{0}, H\right)}^{2}+|g|_{L^{2}\left(\mathbb{R}_{0}, H\right)}^{2}+|\gamma|_{G^{2}\left(\mathbb{R}_{0}, \Gamma\right)}^{2}\right)
\end{aligned}
$$

which, together with (59) gives us

$$
|z|_{L^{2}\left(\mathbb{R}_{0}, H^{1}(\Omega, \mathbb{R})\right)}^{2} \leq \bar{C}_{\left[C_{\mathcal{W}, \mathrm{st}}\right]}\left(\left|z_{0}\right|_{H}^{2}+|z|_{L^{2}\left(\mathbb{R}_{0}, H\right)}^{2}+|g|_{L^{2}\left(\mathbb{R}_{0}, H\right)}^{2}+|\gamma|_{G^{2}\left(\mathbb{R}_{0}, \Gamma\right)}^{2}\right) .
$$

Since $z$ solves system (58), we can obtain that

$$
\left.\left|\partial_{t} z\right|_{L^{2}\left(\mathbb{R}_{0}, H^{-1}(\Omega, \mathbb{R})\right)}^{2} \leq \bar{C}_{\left[C_{\mathcal{W}, \text { st }}\right]}\right]\left.z\right|_{L^{2}\left(\mathbb{R}_{0}, H^{1}(\Omega, \mathbb{R})\right)} ^{2},
$$

which finishes the proof. 
7.3. Null controllability. Consider, in the bounded cylinder $I \times \Omega, I=\left(s_{0}, s_{1}\right)$, the controlled system (57). and also the adjoint system $(16)$.

Let $z(\cdot)=z\left(z_{0}, \zeta\right)(\cdot)$ and $q(\cdot)=q\left(q_{1}\right)(\cdot)$ solve (57) and (16), respectively. Thus, integrating $\frac{\mathrm{d}}{\mathrm{d} t}(z, q)$, we find following the arguments in [Rod14, section 4] and in [Rod15a, section 3.1] that

$$
\begin{aligned}
\left(z\left(s_{1}\right), q_{1}\right)_{H}-\left(z_{0}, q\left(s_{0}\right)\right)_{H} & =\left\langle\mathbf{n} \cdot \nabla q, B_{\Gamma} \zeta\right\rangle_{G^{1}\left(\left(s_{0}, s_{1}\right), \Gamma\right)^{\prime}, G^{1}\left(\left(s_{0}, s_{1}\right), \Gamma\right)} \\
& =\left\langle B_{\Gamma}^{*} \circ(\mathbf{n} \cdot \nabla) q, \zeta\right\rangle_{\mathcal{Z}^{\prime}, \mathcal{Z}}
\end{aligned}
$$

where $B_{\Gamma}^{*} \in \mathcal{L}\left(G^{1}\left(\left(s_{0}, s_{1}\right), \Gamma\right)^{\prime}, \mathcal{Z}^{\prime}\right)$ is the adjoint of $B_{\Gamma}$, and the symbol $\circ$ stands for the composition of two operators.

Also in the boundary case we have the following lemma (cf. Lemma 3.6).

Lemma 7.7. System (16) is $B_{\Gamma}^{*} \circ(\mathbf{n} \cdot \nabla)$ observable in $I$ with

$$
\left|q\left(q_{1}\right)\left(s_{0}\right)\right|_{H} \leq C_{\text {obs }}\left|B_{\Gamma}^{*} \circ(\mathbf{n} \cdot \nabla) q\left(q_{1}\right)\right|_{\mathcal{Z}^{\prime}}
$$

if, and only if, system (57) is null controllable in I and the family of controls $\left\{u\left(z_{0}\right)\right.$ | $\left.z_{0} \in H\right\}$ is a bounded linear function of $z_{0}$ :

$$
\left|\zeta\left(z_{0}\right)\right|_{\mathcal{Z}} \leq C_{\mathrm{obs}}\left|z_{0}\right|_{H} \text {, where } C_{\mathrm{obs}} \text { is as in } 60 \text {. }
$$

Controls supported in a subset. Given an open subset $\Gamma_{\mathrm{c}} \subseteq \Gamma$, we define the spaces

$$
\begin{aligned}
& G_{\mathrm{c}}^{1}\left(\left(s_{0}, s_{1}\right), \Gamma\right):=\left\{\gamma \in G^{1}\left(\left(s_{0}, s_{1}\right), \Gamma\right)|\gamma|_{\Gamma \backslash \overline{\Gamma_{\mathrm{c}}}}=0\right\}, \\
& G_{\mathrm{c}}^{2}\left(\left(s_{0}, s_{1}\right), \Gamma\right):=\left\{\gamma \in G^{2}\left(\left(s_{0}, s_{1}\right), \Gamma\right)|\gamma|_{\Gamma \backslash \overline{\Gamma_{\mathrm{c}}}}=0\right\} .
\end{aligned}
$$

From the results in the section 3.2 and following the arguments in [Rod14, section 4] we have that we can construct open subsets $\tilde{\omega}$ with $\Omega \cap \tilde{\omega}=\emptyset$ and $\Gamma \cap \partial \tilde{\omega}=\overline{\Gamma_{\mathrm{c}}}$ leading to the existence of a constant $C_{\tilde{\omega}, \Omega}>0$, depending on $\tilde{\omega}$ and $\Omega$, such that the weak solution $\tilde{q}$ for 16$)$ in $I \times \widetilde{\Omega}$ with $\widetilde{\Omega}=\Omega \cup \omega \cup \Gamma_{\mathrm{c}}$ ), that is, $\tilde{q}$ solving

$$
\begin{aligned}
& \partial_{t} \tilde{q}-\Delta \tilde{q}+\tilde{a} \tilde{q}-\tilde{b} \cdot \nabla \tilde{q}=0, \\
& \left.\tilde{q}\right|_{\Gamma}=0, \quad \tilde{q}\left(s_{1}\right)=\tilde{q}_{1},
\end{aligned}
$$

satisfies

$$
|\tilde{q}(0)|_{L^{2}(\widetilde{\Omega}, \mathbb{R})}^{2} \leq \mathrm{e}^{C_{\tilde{\omega}, \Omega} \Theta\left(|I|,|a|_{L^{\infty}\left(I, L^{d}\right)},|b|_{L_{w}^{\infty}\left(I, L^{\infty}\right)}, d\right)}|\tilde{q}|_{L^{2}\left(I, L^{2}(\tilde{\omega}, \mathbb{R})\right.}^{2} .
$$

with $\Theta$ as in (18). Here the functions $\tilde{a}$ and $\tilde{b}$ are extensions of $a$ and $b$ by zero outside $\Omega$.

By Lemmas 7.7 and 3.3 we can find a control $\tilde{\eta} \in L^{2}\left(I, L^{2}(\widetilde{\Omega}, \mathbb{R})\right)$ such that the corresponding solution of the system

$$
\begin{aligned}
& \partial_{t} \tilde{z}-\Delta \tilde{z}+\tilde{a} \tilde{z}+\nabla \cdot(\tilde{b} \tilde{z})+1_{\tilde{\omega}} \tilde{\eta}=0, \\
& \left.\tilde{z}\right|_{\tilde{\Gamma}}=0, \quad \tilde{z}\left(s_{0}\right)=\tilde{z}_{0},
\end{aligned}
$$

where $\tilde{z}_{0}$ is the extension of $z_{0}$ by zero outside $\Omega$, satisfies

$$
|\tilde{z}|_{W\left(I, H_{0}^{1}(\widetilde{\Omega}, \mathbb{R}), H^{-1}(\widetilde{\Omega}, \mathbb{R})\right)}^{2} \leq \bar{C}_{\left[|I|, C_{\mathcal{W}}\right]}\left(1+\mathrm{e}^{C_{\tilde{\omega}, \Omega} \Theta}\right)\left|\tilde{z}\left(s_{0}\right)\right|_{L^{2}(\widetilde{\Omega}, \mathbb{R})}^{2},
$$

Therefore $z:=\left.\tilde{z}\right|_{\Omega}$ solves (58) with $\gamma=\left.\tilde{z}\right|_{\Gamma} \in G_{\mathrm{c}}^{1}\left(\left(s_{0}, s_{1}\right), \Gamma\right)$ satisfying

$$
|\gamma|_{G_{c}^{1}\left(\left(s_{0}, s_{1}\right), \Gamma\right)}^{2} \leq C \bar{C}_{\left[|I|, C_{\mathcal{W}}\right]}\left(1+\mathrm{e}^{C_{\tilde{\omega}, \Omega} \Theta}\right)\left|\tilde{z}\left(s_{0}\right)\right|_{L^{2}(\widetilde{\Omega}, \mathbb{R})}^{2} \leq \bar{C}_{\left[|I|, C_{\mathcal{W}}\right]} \mathrm{e}^{C_{\tilde{\omega}, \Omega} \Theta}\left|z\left(s_{0}\right)\right|_{H}^{2} .
$$

Since the choice of such subset $\tilde{\omega}$ is at our disposal, and using Lemmas 3.6 and 7.7 we can conclude that there exists a constant $C_{\Gamma_{c}, \Omega}>0$ depending on $\Gamma_{c}$ and $\Omega$, such that

$$
|q(0)|_{H}^{2} \leq \bar{C}_{\left[|I|, C_{\mathcal{W}}\right]} \mathrm{e}^{\left.C_{\Gamma_{c}, \Omega} \Theta\left(|I|,|a|_{L^{\infty}\left(I, L^{d}\right)},|b|_{L_{w}^{\infty}(I, L} \infty\right), d\right)}\left|1_{\Gamma_{\mathrm{c}}}\left(\mathbf{n} \cdot \nabla q\left(q_{1}\right)\right)\right|_{G_{\mathrm{c}}^{1}\left(\left(s_{0}, s_{1}\right), \Gamma\right)^{\prime}}^{2},
$$


if $(a, b) \in \mathcal{W}$. Further, if $(a, b) \in \mathcal{W}_{\text {st }}$ (cf. [Rod15a, Remark 3.3]) we can derive that

$$
|q(0)|_{H}^{2} \leq \bar{C}_{\left[|I|, C_{\mathcal{W}, \mathrm{st}}\right]} \mathrm{e}^{C_{\Gamma_{c}, \Omega} \Theta\left(|I|,|a|_{L^{\infty}\left(I, L^{d}\right)},|b|_{L_{w}^{\infty}\left(I, L^{\infty}\right)}, d\right)}\left|1_{\Gamma_{\mathrm{c}}}\left(\mathbf{n} \cdot \nabla q\left(q_{1}\right)\right)\right|_{G_{\mathrm{c}}^{2}\left(\left(s_{0}, s_{1}\right), \Gamma\right)^{\prime}}^{2} .
$$

Thus, in the case we take $B_{\Gamma}=\iota_{\Gamma_{\mathrm{c}}} \in \mathcal{L}\left(G_{\mathrm{c}}^{1}\left(\left(s_{0}, s_{1}\right), \Gamma\right), G^{1}\left(\left(s_{0}, s_{1}\right), \Gamma\right)\right), \gamma \mapsto \gamma$, as the inclusion operator, then we have $B_{\Gamma}^{*}=1_{\Gamma_{\mathrm{c}}}$ and we can conclude that 60 holds with $C_{\text {obs }}=C \mathrm{e}^{C_{\Gamma_{c}, \Omega} \Theta\left(|I|,|a|_{L^{\infty}\left(I, L^{d}\right)},|b|_{L_{w}^{\infty}\left(I, L^{\infty}\right)}, d\right)}$. Therefore we have the following.

Theorem 7.8. Let $(a, b) \in \mathcal{W}$ and $B_{\Gamma}=\iota_{\Gamma_{\mathrm{c}}}$. Then, there exists a family $\left\{\bar{\zeta}\left(z_{0}\right) \mid z_{0} \in\right.$ $H\} \subseteq G_{\mathrm{c}}^{1}\left(\left(s_{0}, s_{1}\right), \Gamma\right)$ such that the solutions $z\left(z_{0}, \bar{\zeta}\left(z_{0}\right)\right)$ to (57) satisfy $z\left(z_{0}, \bar{\zeta}\left(z_{0}\right)\right)\left(s_{1}\right)=0$ and, for a constant $\widehat{C}=C\left(\Gamma_{c}, \Omega\right)=C_{\left[C_{\mathcal{W}}\right]}$, we have that

$$
\left|\bar{\zeta}\left(z_{0}\right)\right|_{G_{\mathrm{c}}^{1}\left(\left(s_{0}, s_{1}\right), \Gamma\right)} \leq \bar{C}_{\left[|I|, C_{\mathcal{W}}\right]} \mathrm{e}^{\widehat{C} \Theta}\left|z_{0}\right|_{H},
$$

with $\Theta=\Theta\left(|I|,|a|_{L^{\infty}\left(\mathbb{R}_{0}, L^{d}\right)},|b|_{L_{w}^{\infty}\left(\mathbb{R}_{0}, L^{\infty}\right)}, d\right)$ given by 18$)$.

Furthermore, if $(a, b) \in \mathcal{W}_{\text {st }}$ then there exists a family $\left\{\bar{\zeta}\left(z_{0}\right) \mid z_{0} \in H\right\} \subseteq G_{\mathrm{c}}^{2}\left(\left(s_{0}, s_{1}\right), \Gamma\right)$ such that, still $z\left(z_{0}, \bar{\zeta}\left(z_{0}\right)\right)\left(s_{1}\right)=0$, and we have

$$
\left|\bar{\zeta}\left(z_{0}\right)\right|_{G_{\mathrm{c}}^{2}\left(\left(s_{0}, s_{1}\right), \Gamma\right)} \leq \bar{C}_{\left[|I|, C_{\mathcal{W}, \mathrm{st}}\right]} \mathrm{e}^{\widehat{C} \Theta}\left|z_{0}\right|_{H} .
$$

Given a nonzero smooth function $\chi_{\Gamma}: \Gamma \rightarrow \mathbb{R}$ with supp $\chi_{\Gamma} \subseteq \overline{\Gamma_{\mathrm{c}}}$, we also have the following (cf. [Rod15a, section 3.3]).

Corollary 7.9. Theorem 7.8 holds in the more general case

$$
B_{\Gamma}=1_{\Gamma_{\mathrm{c}}} \chi_{\Gamma} 1_{\Gamma_{\mathrm{c}}} \in \mathcal{L}\left(G_{\mathrm{c}}^{1}\left(\left(s_{0}, s_{1}\right), \Gamma\right), G^{1}\left(\left(s_{0}, s_{1}\right), \Gamma\right)\right),
$$

with $\widehat{D}=\widehat{D}\left(\chi_{\Gamma}, \Gamma_{\mathrm{c}}, \Omega\right)>0$ in the place of $\widehat{C}$.

7.4. Stabilization to zero by finite dimensional controls. Now we consider the case of finite dimensional controls, of the form $\sum_{i=1}^{M} u_{i}(t) \Phi_{i}(x)$. Let us consider a family $\widehat{\mathcal{C}_{\Gamma}}=$ $\left\{\widehat{\Psi}_{i} \in H^{\frac{3}{2}}(\Gamma, \mathbb{R}) \mid i \in\{1,2, \ldots, M\}\right\}$ satisfying $1_{\Gamma_{\mathrm{c}}} \chi_{\Gamma} \widehat{\mathcal{C}}_{\Gamma} \subset H^{\frac{3}{2}}(\Gamma, \mathbb{R})$, and denote by $P_{M}$ the orthogonal projection in $L^{2}(\Gamma, \mathbb{R})$ onto $\mathcal{S}_{\widehat{\mathcal{C}}_{\Gamma}}:=\operatorname{span} \widehat{\mathcal{C}}_{\Gamma}$.

Let us also fix a positive constant $\bar{\lambda}>0$ and consider, in $\mathbb{R}_{s_{0}} \times \Omega$, the system:

$$
\begin{aligned}
& \partial_{t} z_{\bar{\lambda}}-\Delta z_{\bar{\lambda}}+\left(a-\frac{\bar{\lambda}}{2}\right) z_{\bar{\lambda}}+\nabla \cdot\left(b z_{\bar{\lambda}}\right)=0, \\
& \left.z_{\bar{\lambda}}\right|_{\Gamma}=1_{\Gamma_{\mathrm{c}}} \chi_{\Gamma} P_{M} 1_{\Gamma_{\mathrm{c}}} \zeta, \quad z_{\bar{\lambda}}\left(s_{0}\right)=z_{0} .
\end{aligned}
$$

Definition 7.10. We say that $(62)$ is exponentially stabilizable to zero, with rate 0 , if there are constants $C_{1}>0$ and $C_{2}>0$, and a bounded family $\left\{\zeta=\zeta\left(z_{0}\right) \mid z_{0} \in H\right\} \subseteq$ $G_{\mathrm{c}}^{1}\left(\mathbb{R}_{0}, \Gamma\right)$

$$
\left|\zeta\left(z_{0}\right)\right|_{G_{\mathrm{c}}^{1}\left(\mathbb{R}_{0}, \Gamma\right)}^{2} \leq C_{1}\left|z_{0}\right|_{H}^{2}
$$

such that the corresponding global solution $z_{\bar{\lambda}}(t)=z_{\bar{\lambda}}\left(z_{0}, \zeta\left(z_{0}\right)\right)(t)$ satisfies

$$
\left|z_{\bar{\lambda}}(t)\right|_{H}^{2} \leq C_{2}\left|z_{0}\right|_{H}^{2}, \quad \text { for all } t \geq s_{0}
$$

Notice that we may write

$$
1_{\Gamma_{\mathrm{c}}} \chi_{\Gamma} P_{M} 1_{\Gamma_{\mathrm{c}}} \zeta=1_{\Gamma_{\mathrm{c}}} \chi_{\Gamma} \sum_{i=1}^{M} \zeta_{i} \widehat{\Psi}_{i}=\sum_{i=1}^{M} u_{i} \Psi_{i}
$$

with $u_{i}:=\zeta_{i}$ and $\Psi_{i}:=1_{\Gamma} \chi_{\Gamma} \widehat{\Psi}_{i}, i \in\{1,2, \ldots, M\}$.

Henceforth we use the control operator

$$
B_{M}^{\Gamma}:=1_{\Gamma_{\mathrm{c}}} \chi_{\Gamma} P_{M} 1_{\Gamma_{\mathrm{c}}} .
$$


Further $\Theta$ and $\widehat{D}$ are as in Theorem 7.8 and Corollary 7.9 .

Let $z_{\bar{\lambda}}$ solve (57) with $B_{\Gamma}=1_{\Gamma_{\mathrm{c}}} \chi_{\Gamma} 1_{\Gamma_{\mathrm{c}}}$ and $\left(a-\frac{\bar{\lambda}}{2}\right)$ in the place of $a$, and with the corresponding control $\zeta=\bar{\zeta}\left(z_{0}\right) \in G_{\mathrm{c}}^{2}\left(\left(s_{0}, s_{1}\right), \Gamma\right)$ given by Corollary 7.9 , and let $z_{M}$ solve (62) also with $\zeta=\bar{\zeta}\left(z_{0}\right)$. Then, $d=z-z_{M}$ solves

$$
\begin{aligned}
& \partial_{t} d-\Delta d+\left(a-\frac{\bar{\lambda}}{2}\right) d+\nabla \cdot(b d)=0, \\
& \left.d\right|_{\Gamma}=1_{\Gamma_{\mathrm{c}}} \chi_{\Gamma}\left(1-P_{M}\right) 1_{\Gamma_{\mathrm{c}}} \zeta\left(z_{0}\right), \quad d\left(s_{0}\right)=0 .
\end{aligned}
$$

If $(a, b) \in \mathcal{W}_{\text {st }}$ satisfies 46 , from Lemma 7.2 and Corollary 7.9 it follows

$$
|d|_{W\left(I, H^{1}(\Omega, \mathbb{R}), V^{\prime}\right)}^{2} \leq \Xi_{\Gamma}(|I|)\left|1_{\Gamma_{\mathrm{c}}} \chi_{\Gamma}\left(1-P_{M}\right) 1_{\Gamma_{\mathrm{c}}}\right|_{\mathcal{L}\left(G_{\mathrm{c}}^{2}\left(\left(s_{0}, s_{1}\right), \Gamma\right)\right), G_{\mathrm{c}}^{1}\left(\left(s_{0}, s_{1}\right), \Gamma\right)}^{2}\left|z_{0}\right|_{H}^{2}
$$

with

$$
\Xi_{\Gamma}(\tau):=\bar{C}_{\left[|I|, C_{\mathcal{W}, \mathrm{st}}\right]} \mathrm{e}^{\widehat{D} \Theta\left(\tau,\left|a-\frac{\bar{\lambda}}{2}\right|_{L^{\infty}\left(\mathbb{R}_{0}, L^{d}\right)},|b|_{L_{w}^{\infty}\left(\mathbb{R}_{0}, L^{\infty}\right)}, d\right)}, \quad \tau>0 .
$$

We can see that when $\left(a-\frac{\bar{\lambda}}{2}, b\right)=(0,0)$ then 62$)$ is exponentially stabilizable to zero, with rate 0 , just by setting $\zeta\left(z_{0}\right)=0$ for all $z_{0} \in H$. Therefore from now we consider the case $\left(a-\frac{\bar{\lambda}}{2}, b\right) \neq(0,0)$ where we can see that it holds

$$
\lim _{\tau \rightarrow+\infty} \Xi_{\Gamma}(\tau)=+\infty \text { and } \lim _{\tau \rightarrow 0} \Xi_{\Gamma}(\tau)=+\infty .
$$

Hence we can set $T_{*}>0$ such that $\Xi_{\Gamma}\left(T_{*}\right)=\min _{\tau>0} \Xi_{\Gamma}(\tau)=: \Upsilon_{\Gamma}$.

This allow us to derive the following result on a sufficient condition on the family $\widehat{\mathcal{C}}_{\Gamma}$ for the existence of a stabilizing control. The proof can be done following the arguments used in the internal controls case as in in [KR15a, BKR15].

Theorem 7.11. Let us be given a nonzero $\chi_{\Gamma} \in C^{\infty}(\bar{\Omega})$ satisfying $\operatorname{supp} \chi_{\Gamma} \subseteq \overline{\Gamma_{\mathrm{c}}}$. If

$$
\left|1_{\Gamma_{\mathrm{c}}} \chi_{\Gamma}\left(1-P_{M}\right) 1_{\Gamma_{\mathrm{c}}}\right|_{\mathcal{L}\left(G_{\mathrm{c}}^{2}\left(\left(s_{0}, s_{1}\right), \Gamma\right)\right), G_{\mathrm{c}}^{1}\left(\left(s_{0}, s_{1}\right), \Gamma\right)}^{2} \leq \Upsilon_{\Gamma}^{-1}
$$

then system (62) is stabilizable to zero with rate 0.

7.5. Feedback stabilizing rule and Riccati equation. Here we follow the ideas from [Bad09, Rod15b], by considering a suitable extended system. This is done in order to be able to use the Dynamical Programming Principle.

Here we suppose that (64) holds true, so that from Theorem 7.11 we know that there exists $\zeta \in G_{\mathrm{c}}^{2}\left(\left(s_{0}, s_{1}\right), \Gamma\right)$ stabilizing system 62 to zero with rate 0 . Writing

$$
1_{\Gamma_{\mathrm{c}}} \chi_{\Gamma} P_{M} 1_{\Gamma_{\mathrm{c}}} \zeta=\sum_{i=1}^{M} u_{i} 1_{\Gamma_{\mathrm{c}}} \chi_{\Gamma} \widehat{\Psi}_{i}
$$

we know that $E_{2} 1_{\Gamma_{\mathrm{c}}} \chi_{\Gamma} P_{M} 1_{\Gamma_{\mathrm{c}}} \zeta \in W^{1}\left(\left(s_{0}, s_{1}\right), H^{2}(\Omega, \mathbb{R}), H\right)$, but we do not have necessarily that $u_{i} \in H^{1}\left(\left(s_{0}, s_{1}\right), \mathbb{R}\right)$, for each $i \in\{1,2, \ldots, M\}$. Though, we can say that $u_{i} \in$ $H^{\frac{3}{4}}\left(\left(s_{0}, s_{1}\right), \mathbb{R}\right)$, see [LM72b, chapter 4 , section 2.2$]$.

Since we would like to follow the procedure in $\left[\operatorname{Rod} 15 \mathrm{~b}\right.$ ] we need $u_{i} \in H^{1}\left(\left(s_{0}, s_{1}\right), \mathbb{R}\right)$. This regularity in time could be guaranteed (for a suitable class of actuators) by following the arguments based on suitable truncated observability inequalities, in both space and time variable, from [Rod15a, section 4.3], see also [Shi11]. Skiping those technical details, we would arrive to the following result for a suitable projection $P_{\tilde{M}}^{t}$ defined in $L^{2}\left(\left(s_{0}, s_{1}\right), \mathbb{R}\right)$, with range contained in $H^{1}\left(\left(s_{0}, s_{1}\right), \mathbb{R}\right)$.

Proposition 7.12. Let us be given $\chi_{\Gamma}$ as in Theorem 7.11. If

$$
\left|1_{\Gamma_{\mathrm{c}}} \chi_{\Gamma}\left(1-P_{\tilde{M}}^{t} P_{M}\right) 1_{\Gamma_{\mathrm{c}}}\right|_{\mathcal{L}\left(G_{\mathrm{c}}^{2}\left(\left(s_{0}, s_{1}\right), \Gamma\right)\right), G_{\mathrm{c}}^{1}\left(\left(s_{0}, s_{1}\right), \Gamma\right)}^{2} \leq \Upsilon_{\Gamma}^{-1},
$$

then system (62), with $P_{\tilde{M}}^{t} P_{M}$ in the place of $P_{M}$, is stabilizable to zero with rate 0 . 
Remark 7.13. A characterization of $G_{\mathrm{c}}^{2}\left(\left(s_{0}, s_{1}\right), \Gamma\right)$ in terms of fractional Sobolev spaces can be found in LM72b, chapter 4, section 2.2]. However, we do not know a similar characterization of $G_{\mathrm{c}}^{1}\left(\left(s_{0}, s_{1}\right), \Gamma\right)$. Thus, we cannot follow word by word the procedure in [Rod15a, section 4.3], but we can use the main idea. Starting by writing $1-P_{\tilde{M}}^{t} P_{M}=$ $1-P_{M}+\left(1-P_{\tilde{M}}^{t}\right) P_{M}$, we find (using the identity $P_{M}=P_{M} P_{M}$ )

$$
\begin{aligned}
& \quad\left|1_{\Gamma_{\mathrm{c}}} \chi_{\Gamma}\left(1-P_{\tilde{M}}^{t} P_{M}\right) 1_{\Gamma_{\mathrm{c}}}\right|_{\mathcal{L}\left(G_{\mathrm{c}}^{2}\left(\left(s_{0}, s_{1}\right), \Gamma\right)\right), G_{\mathrm{c}}^{1}\left(\left(s_{0}, s_{1}\right), \Gamma\right)} \\
& \leq\left|1_{\Gamma_{\mathrm{c}}} \chi\left(1-P_{M}\right) 1_{\Gamma_{\mathrm{c}}}\right|_{\mathcal{L}\left(G_{\mathrm{c}}^{2}\left(\left(s_{0}, s_{1}\right), \Gamma\right)\right), G_{\mathrm{c}}^{1}\left(\left(s_{0}, s_{1}\right), \Gamma\right)} \\
& \quad+\left|1_{\Gamma_{\mathrm{c}}} \chi\left(1-P_{\tilde{M}}^{t}\right) P_{M}\right|_{\mathcal{L}\left(G_{\mathrm{c}}^{2}\left(\left(s_{0}, s_{1}\right), \Gamma\right)\right), G_{\mathrm{c}}^{\frac{3}{2}}\left(\left(s_{0}, s_{1}\right), \Gamma\right)}\left|P_{M} 1_{\Gamma_{\mathrm{c}}}\right|_{\mathcal{L}\left(G_{\mathrm{c}}^{\frac{3}{2}}\left(\left(s_{0}, s_{1}\right), \Gamma\right)\right), G_{\mathrm{c}}^{1}\left(\left(s_{0}, s_{1}\right), \Gamma\right)} \\
& \leq\left|1_{\Gamma_{\mathrm{c}}} \chi\left(1-P_{M}\right) 1_{\Gamma_{\mathrm{c}}}\right|_{\mathcal{L}\left(G_{\mathrm{c}}^{2}\left(\left(s_{0}, s_{1}\right), \Gamma\right)\right), G_{\mathrm{c}}^{1}\left(\left(s_{0}, s_{1}\right), \Gamma\right)},\left|P_{M} 1_{\Gamma_{\mathrm{c}}}\right|_{\mathcal{L}\left(G_{\mathrm{c}}^{\frac{3}{2}}\left(\left(s_{0}, s_{1}\right), \Gamma\right)\right), G_{\mathrm{c}}^{1}\left(\left(s_{0}, s_{1}\right), \Gamma\right)}, \\
& \quad+C_{\chi_{\Gamma}, M}\left|\left(1-P_{\tilde{M}}^{t}\right)\right|_{\left.\left.\mathcal{L}\left(H^{\frac{3}{4}}\left(\left(s_{0}, s_{1}\right), \mathbb{R}\right)\right), H^{\frac{2}{3}}\left(\left(s_{0}, s_{1}\right), \mathbb{R}\right)\right)\right)}
\end{aligned}
$$

where $G_{\mathrm{c}}^{\frac{3}{2}}\left(\left(s_{0}, s_{1}\right), \Gamma\right):=\left[G_{\mathrm{c}}^{2}\left(\left(s_{0}, s_{1}\right), \Gamma\right), G_{\mathrm{c}}^{1}\left(\left(s_{0}, s_{1}\right), \Gamma\right)\right]_{\frac{1}{2}}$ is an interpolation space in the sense of [LM72a]. If $P_{M}$ satisfies (64) with, say, $\frac{\Upsilon_{\Gamma}^{-1}}{2}$ in the place of $\Upsilon_{\Gamma}^{-1}$, then we can set $P_{\tilde{M}}^{t}$ (i.e., set $\tilde{M}$ big enough) such that 65 holds true. Finally, notice that with $X=W^{1}\left(\left(s_{0}, s_{1}\right), H^{2}(\Omega, \mathbb{R}), H\right)$ and $Y=W^{1}\left(\left(s_{0}, s_{1}\right), H^{1}(\Omega, \mathbb{R}), H^{-1}(\Omega, \mathbb{R})\right)$ we have (using some results from [LM72b, chapter 4 , section 2.2])

$$
\begin{aligned}
& G_{\mathrm{c}}^{\frac{3}{2}}\left(\left(s_{0}, s_{1}\right), \Gamma\right)=\left.[X, Y]_{\frac{1}{2}}\right|_{\Gamma}, \\
& G_{\mathrm{c}}^{2}\left(\left(s_{0}, s_{1}\right), \Gamma\right) \stackrel{\mathrm{c}}{\hookrightarrow} G_{\mathrm{c}}^{\frac{3}{2}}\left(\left(s_{0}, s_{1}\right), \Gamma\right) \stackrel{\mathrm{c}}{\hookrightarrow} G_{\mathrm{c}}^{1}\left(\left(s_{0}, s_{1}\right), \Gamma\right), \\
&\left.W^{1}\left(\left(s_{0}, s_{1}\right), H^{\frac{3}{2}}(\Omega, \mathbb{R}), H\right)\right|_{\Gamma}=L^{2}\left(\left(s_{0}, s_{1}\right), H^{1}(\Gamma, \mathbb{R})\right) \cap H^{\frac{2}{3}}\left(\left(s_{0}, s_{1}\right), L^{2}(\Gamma, \mathbb{R})\right), \\
&\left.W^{1}\left(\left(s_{0}, s_{1}\right), H^{\frac{3}{2}}(\Omega, \mathbb{R}), H\right)\right|_{\Gamma}=\left.\left[X, W^{1}\left(\left(s_{0}, s_{1}\right), H^{1}(\Omega, \mathbb{R}), H\right)\right]_{\frac{1}{2}}\right|_{\Gamma} \\
& \hookrightarrow G_{\mathrm{c}}^{\frac{3}{2}}\left(\left(s_{0}, s_{1}\right), \Gamma\right), \\
& H^{\frac{3}{4}}\left(\left(s_{0}, s_{1}\right), \mathbb{R}\right) \stackrel{\mathrm{c}}{\hookrightarrow} H^{\frac{2}{3}}\left(\left(s_{0}, s_{1}\right), \mathbb{R}\right) .
\end{aligned}
$$

7.5.1. The auxiliary extended system. Once we have that $u_{i} \in H^{1}\left(\left(s_{0}, s_{1}\right), \mathbb{R}\right)$, for each $i \in\{1,2, \ldots, M\}$, then we can rewrite 62$)$ in the variables $\left(y_{\bar{\lambda}}, \kappa_{\bar{\lambda}}\right)=\left(z_{\bar{\lambda}}-B_{\Psi} \kappa_{\bar{\lambda}}, \kappa_{\bar{\lambda}}\right)$, as the extended system

$$
\begin{aligned}
& \partial_{t} y_{\bar{\lambda}}-\Delta y_{\bar{\lambda}}+\left(a-\frac{\bar{\lambda}}{2}\right) y_{\bar{\lambda}}+\nabla \cdot\left(b y_{\bar{\lambda}}\right)-\Delta B_{\Psi} \kappa_{\bar{\lambda}}+a B_{\Psi} \kappa_{\bar{\lambda}}+\nabla \cdot\left(b B_{\Psi} \kappa_{\bar{\lambda}}\right)+\bar{\varsigma} B_{\Psi} \kappa_{\bar{\lambda}}=B_{\Psi} \varkappa_{\bar{\lambda}}, \\
& \partial_{t} \kappa_{\bar{\lambda}}-\frac{\bar{\lambda}}{2} \kappa_{\bar{\lambda}}+\bar{\zeta} \kappa_{\bar{\lambda}}=\varkappa_{\bar{\lambda}}, \\
& y_{\bar{\lambda}}\left(s_{0}\right)=y_{0}=z_{0}-B_{\Psi} \kappa_{0}, \quad \kappa_{\bar{\lambda}}\left(s_{0}\right)=\kappa_{0},\left.\quad y_{\bar{\lambda}}\right|_{\Gamma}=0
\end{aligned}
$$

where $B_{\Psi}: \mathbb{R}^{M} \rightarrow H^{2}(\Omega, \mathbb{R})$ is given by

$$
B_{\Psi} \kappa:=\sum_{i=1}^{M} \kappa_{i} \widetilde{\Psi}_{i}, \quad \text { with } \quad \widetilde{\Psi}_{i} \in H^{2}(\Omega, \mathbb{R}),\left.\quad \widetilde{\Psi}_{i}\right|_{\Gamma}=\Psi_{i}:=1_{\Gamma_{\mathrm{c}}} \chi_{\Gamma} \widehat{\Psi}_{i}
$$

where the extensions $\widetilde{\Psi}_{i}$ of the $\Psi_{i}$ s are fixed. Recall that by assumption $\Psi_{i} \in H^{\frac{3}{2}}(\Gamma, \mathbb{R})=$ $\left.H^{2}(\Omega, \mathbb{R})\right|_{\Gamma}$ for all $i \in\{1,2, \ldots, M\}$. Furthermore, $\bar{\varsigma} \in \mathbb{R}$ is a parameter at our disposal, notice that it does not appear in 62 .

From Proposition 7.12 we can conclude that there exists $\kappa=u \in H^{1}\left(\left(s_{0}, s_{1}\right), \mathbb{R}^{M}\right)$ such that system (66) is stabilizable to zero with rate 0 , provided 65 holds true. 
Hereafter we will use a particular extension, namely, the given actuators $\Psi_{i}$, defined on the boundary $\Gamma$, are extended to $\widetilde{\Psi}_{i}$, defined in $\Omega$, by solving the elliptic system

$$
-\Delta \widetilde{\Psi}+{ }_{\zeta} \widetilde{\Psi}_{i}=0,\left.\quad \widetilde{\Psi}_{i}\right|_{\Gamma}=\Psi_{i} .
$$

The following discretization is valid only for this particular extension, which as we see leads to suitable simplifications on the corresponding Riccati equations, namely the 2nd order space derivatives term $-\Delta B_{\widetilde{\Psi}} \kappa$ disappears in (66), because $\left(A^{a, b}+\bar{\varsigma}\right) B_{\widetilde{\Psi}}$ reduces to $a B_{\widetilde{\Psi}}+\nabla \cdot\left(b B_{\widetilde{\Psi}}\right)$. To simplify the exposition, we rewrite system $\{(66),(68)\}$, for $(y, \kappa) \in H \times \mathbb{R}^{M}$, as

$$
\partial_{t}\left[\begin{array}{c}
y_{\bar{\lambda}} \\
\kappa_{\bar{\lambda}}
\end{array}\right]+\left[\begin{array}{cc}
-\Delta+K^{a, b, \bar{\lambda}} & K^{a, b, 0} \\
0 & \bar{\varsigma}_{\bar{\lambda}}
\end{array}\right]\left[\begin{array}{c}
y_{\bar{\lambda}} \\
\kappa_{\bar{\lambda}}
\end{array}\right]-\left[\begin{array}{c}
B_{\Psi} \\
1
\end{array}\right] \varkappa_{\bar{\lambda}}=0, \quad\left[\begin{array}{l}
y_{\bar{\lambda}}\left(s_{0}\right) \\
\kappa_{\bar{\lambda}}\left(s_{0}\right)
\end{array}\right]=\left[\begin{array}{l}
y_{0} \\
\kappa_{0}
\end{array}\right],
$$

where $\bar{\varsigma}_{r}$ and $K^{a, b, r} \in \mathcal{L}\left(V+B_{\Psi} \mathbb{R}^{M}, V^{\prime}\right)$, for $r \geq 0$, are given by

$$
\bar{\varsigma}_{r}:=\bar{\varsigma}-\frac{r}{2}, \quad K^{a, b, r} w:=a w+\nabla \cdot(b w)-\frac{r}{2} .
$$

As in the internal case we can prove that the control can be taken in feedback form,

$$
\varkappa_{\bar{\lambda}}=\mathcal{F}_{\bar{\lambda}}(t)\left(y_{\bar{\lambda}}, \kappa_{\bar{\lambda}}\right)=-B_{M}^{\Gamma} \Pi_{\bar{\lambda}}^{\Gamma}(t)\left(y_{\bar{\lambda}}, \kappa_{\bar{\lambda}}\right),
$$

with $B_{M}^{\Gamma}=\left[\begin{array}{c}B_{\Psi} \\ 1\end{array}\right]$, with adjoint $B_{M}^{\Gamma *}=\left[\begin{array}{ll}B_{\Psi}^{*} & 1\end{array}\right]$. Furthermore the operator $\Pi_{\bar{\lambda}}^{\Gamma}$ can be chosen to satisfy a differential Riccati equation

$$
\frac{\mathrm{d}}{\mathrm{d} t} \Pi_{\bar{\lambda}}^{\Gamma}+\Pi_{\bar{\lambda}}^{\Gamma} \mathbb{A}_{\bar{\lambda}, \bar{\varsigma}}^{a, b}+\mathbb{A}_{\bar{\lambda}, \bar{\varsigma}}^{a, b^{*}} \Pi_{\bar{\lambda}}^{\Gamma}-\Pi_{\bar{\lambda}}^{\Gamma} B_{M}^{\Gamma} \mathcal{R}^{-1} B_{M}^{\Gamma *} \Pi_{\bar{\lambda}}^{\Gamma}+\mathcal{M}^{*} \mathcal{M}=0
$$

with

$$
\mathbb{A}_{\bar{\lambda}, \bar{\varsigma}}^{a, b}=-\left[\begin{array}{cc}
-\Delta+K^{a, b, \bar{\lambda}} & K^{a, b, 0} \\
0 & \overline{\varsigma_{\bar{\lambda}}}
\end{array}\right],
$$

and for suitable $\mathcal{R} \in \mathcal{L}\left(\mathbb{R}^{M}\right)$ and $\mathcal{M} \in \mathcal{L}\left(V \times \mathbb{R}^{M} \rightarrow V^{\prime} \times \mathbb{R}^{M}\right)$. In this case, the obtained feedback control is the one that minimizes

$$
\int_{s_{0}}^{+\infty}\left|\mathcal{M}\left[\begin{array}{l}
y_{\bar{\lambda}}(\tau) \\
\kappa_{\bar{\lambda}}(\tau)
\end{array}\right]\right|_{H \times \mathbb{R}^{M}}^{2}+\varkappa_{\bar{\lambda}}(\tau)^{\top} \mathcal{R} \varkappa_{\bar{\lambda}}(\tau) \mathrm{d} \tau
$$

For example we can take $\mathcal{R}=1$ and $\mathcal{M}=\left[\begin{array}{cc}(-\Delta)^{\frac{1}{2}} & 0 \\ 0 & 1\end{array}\right]$ as in [BRS11, KR15b]. From Lemma 7.6 we can also take $\mathcal{M}=\left[\begin{array}{ll}1 & 0 \\ 0 & 1\end{array}\right]$ instead (cf. [Rod15b, BKR15]).

It follows (cf. Theorem 3.15 and Corollary 3.16) that the solution $\left(y_{\bar{\lambda}}, \kappa_{\bar{\lambda}}\right)$ of the system

$$
\partial_{t}\left[\begin{array}{l}
y_{\bar{\lambda}} \\
\kappa_{\bar{\lambda}}
\end{array}\right]-\mathbb{A}_{\bar{\lambda}, \bar{\varsigma}}^{a, b}\left[\begin{array}{c}
y_{\bar{\lambda}} \\
\kappa_{\bar{\lambda}}
\end{array}\right]+\left[\begin{array}{c}
B_{\Psi} \\
1
\end{array}\right]\left[\begin{array}{cc}
B_{\Psi}^{*} & 1]
\end{array} \Pi_{\bar{\lambda}}^{\Gamma}\left[\begin{array}{l}
y_{\bar{\lambda}} \\
\kappa_{\bar{\lambda}}
\end{array}\right]=0, \quad\left[\begin{array}{c}
y_{\bar{\lambda}}\left(s_{0}\right) \\
\kappa_{\bar{\lambda}}\left(s_{0}\right)
\end{array}\right]=\left[\begin{array}{l}
y_{0} \\
\kappa_{0}
\end{array}\right],\right.
$$

remains bounded: $\left|\left(y_{\bar{\lambda}}, \kappa_{\bar{\lambda}}\right)\right|_{H \times \mathbb{R}^{M}}^{2} \leq \bar{C}_{\left[C_{\mathcal{W}}, \bar{\lambda}, \frac{1}{\lambda}\right]} \mid\left(y_{0},\left.\kappa_{0}\right|_{H \times \mathbb{R}^{M}} ^{2}\right.$. Therefore, the solution of

$$
\partial_{t}\left[\begin{array}{l}
y \\
\kappa
\end{array}\right]+\mathbb{A}_{0, \bar{\varsigma}}^{a, b}\left[\begin{array}{c}
y \\
\kappa
\end{array}\right]+\left[\begin{array}{c}
B_{\Psi} \\
1
\end{array}\right]\left[\begin{array}{ll}
B_{\Psi}^{*} & 1
\end{array}\right] \Pi_{\bar{\lambda}}^{\Gamma}\left[\begin{array}{l}
y \\
\kappa
\end{array}\right]=0, \quad\left[\begin{array}{c}
y\left(s_{0}\right) \\
\kappa\left(s_{0}\right)
\end{array}\right]=\mathrm{e}^{-\frac{\bar{\lambda}}{2} s_{0}}\left[\begin{array}{l}
y_{0} \\
\kappa_{0}
\end{array}\right],
$$

goes exponentially to 0 with rate $\frac{\bar{\lambda}}{2}$. Notice that $\left(y_{\bar{\lambda}}, \kappa_{\bar{\lambda}}\right)$ solves (71) if, and only if, $(y, \kappa)=\mathrm{e}^{-\frac{\bar{\lambda}}{2} t}\left(y_{\bar{\lambda}}, \kappa_{\bar{\lambda}}\right)$ solves 72 . 
7.5.2. From the auxiliary to the original system. Once we have the feedback rule

$$
\Pi_{\bar{\lambda}}^{\Gamma}=:\left[\begin{array}{cc}
\Pi_{1,1}^{\Gamma, \bar{\lambda}} & \Pi_{1,2}^{\Gamma, \bar{\lambda}} \\
\Pi_{1,2}^{\Gamma, \bar{\lambda}^{*}} & \Pi_{2,2}^{\Gamma, \bar{\lambda}}
\end{array}\right] \in \mathcal{L}\left(H \times \mathbb{R}^{M}\right)
$$

we observe that

$$
\varkappa=-\left[\begin{array}{ll}
B_{\Psi}^{*} & 1
\end{array}\right] \Pi_{\bar{\lambda}}^{\Gamma}\left[\begin{array}{l}
y \\
\kappa
\end{array}\right]=-\left(B_{\Psi}^{*} \Pi_{1,1}^{\Gamma, \bar{\lambda}}+\Pi_{1,2}^{\Gamma, \bar{\lambda}^{*}}\right) y-\left(B_{\Psi}^{*} \Pi_{1,2}^{\Gamma, \bar{\lambda}}+\Pi_{2,2}^{\Gamma, \bar{\lambda}}\right) \kappa,
$$

and that $z_{\bar{\lambda}}=y_{\bar{\lambda}}+B_{\Psi} \kappa_{\bar{\lambda}}$ solves for time $t \geq s_{0}$

$$
\begin{aligned}
& \partial_{t} z_{\bar{\lambda}}-\Delta z_{\bar{\lambda}}+\left(a-\frac{\bar{\lambda}}{2}\right) z_{\bar{\lambda}}+\nabla \cdot\left(b z_{\bar{\lambda}}\right)=0, \quad z_{\bar{\lambda}}\left(s_{0}\right)=y_{0}+B_{\Psi} \kappa_{0}, \\
& \left.z_{\bar{\lambda}}\right|_{\Gamma}=B_{\Psi}^{\Gamma}\left(\mathrm{e}^{-\bar{\varsigma} t} \kappa_{0}-\int_{s_{0}}^{\cdot} \mathrm{e}^{-\bar{\varsigma}(\cdot-s)}\left[\begin{array}{ll}
B_{\Psi}^{*} & 1
\end{array}\right] \Pi_{\bar{\lambda}}^{\Gamma}(s)\left[\begin{array}{l}
y_{\bar{\lambda}}(s) \\
\kappa_{\bar{\lambda}}(s)
\end{array}\right] \mathrm{d} s\right),
\end{aligned}
$$

and remains bounded. Here $B_{\Psi}^{\Gamma}: \mathbb{R}^{M} \rightarrow \operatorname{span} 1_{\Gamma_{\mathrm{c}}} \chi_{\Gamma} \widehat{\mathcal{C}}_{\Gamma}$ stands for the mapping

$$
B_{\Psi}^{\Gamma} u \mapsto \sum_{i=1}^{M} u_{i} 1_{\Gamma_{\mathrm{c}}} \chi_{\Gamma} \widehat{\Psi}_{i} .
$$

Notice that, without loss of generality, we can suppose that the functions $\Psi_{i}$ are linearly independent. In this case, we have that $V \cap B_{\Psi} \mathbb{R}^{M}=\emptyset$. Thus, since for a.e. $t \geq s_{0}$ we have $z(t) \in V+B_{\Psi} \mathbb{R}^{M}$, we can write $z(t)=y^{z}(t)+B_{\Psi} \kappa^{z}(t)=0$ in a unique way, with $\left(y^{z}(t), \kappa^{z}(t)\right) \in V \times \mathbb{R}^{M}$. That is, $y_{\bar{\lambda}}(s)$ and $\kappa_{\bar{\lambda}}(s)$ appearing in (73) can be constructed from $z_{\bar{\lambda}}(s)$.

A procedure to construct the mapping $z \mapsto\left(y^{z}, \kappa^{z}\right)$ is the following: we orthonormalize (e.g., by applying Gram-Schmidt procedure) the family $\widetilde{\Psi}_{i}$, say in the $H$-scalar product. In this way we arrive to the orthonormal family $\breve{\mathcal{C}}=\left\{\breve{\Psi}_{i} \mid i \in\{1,2, \ldots, M\}\right\}$, Now we can write $v=w+\sum_{i=1}^{M} \xi_{i} \breve{\Psi}_{i}$, with $\xi_{i}:=\left(v, \breve{\Psi}_{i}\right)_{H}$. Finally, we construct $\sigma$ by a matrix of change of coordinates $\sum_{i=1}^{M} \sigma_{i} \widetilde{\Psi}_{i}:=\sum_{i=1}^{M} \xi_{i} \breve{\Psi}_{i}$, and still have $v=w+\sum_{i=1}^{M} \sigma_{i} \widetilde{\Psi}_{i}$. That is, denoting $y^{z}=w$ and $\kappa^{z}=\sigma$, the mapping $z(t) \mapsto\left(y^{z(t)}, \kappa^{z(t)}\right)$ is well defined and we can rewrite the integral feedback rule in (73) as

$$
\left.z\right|_{\Gamma}=B_{\Psi}^{\Gamma}\left(\mathrm{e}^{-\bar{\varsigma} t} \kappa_{0}-\int_{s_{0}}^{\cdot} \mathrm{e}^{-\bar{\varsigma}(\cdot-s)}\left[\begin{array}{ll}
B_{\Psi}^{*} & 1
\end{array}\right] \Pi_{\bar{\lambda}}^{\Gamma}(s)\left[\begin{array}{l}
y^{z(s)} \\
\kappa^{z(s)}
\end{array}\right] \mathrm{d} s\right),
$$

which underlines the (integral) feedback nature of the control, also as a boundary control.

7.6. The nonlinear systems. As we have done for the case of internal controls, under suitable conditions on the nonlinear function $\mathcal{N}$ we can derive also that the local stabilization result holds for nonlinear system in the form $(73)$ with $\mathcal{N}$ as a perturbation

$$
\begin{aligned}
& \partial_{t} z_{\bar{\lambda}}+A^{a, b} z_{\bar{\lambda}}-\frac{\bar{\lambda}}{2} z_{\bar{\lambda}}=\mathcal{N}\left(z_{\bar{\lambda}}\right),\left.\quad z_{\bar{\lambda}}\right|_{\Gamma}=B_{\Psi}^{\Gamma} \kappa_{\bar{\lambda}}, \quad z_{\bar{\lambda}}\left(s_{0}\right)=y_{\bar{\lambda}}\left(s_{0}\right)+B_{\Psi} \kappa_{\bar{\lambda}}\left(s_{0}\right), \\
& \partial_{t} \kappa_{\bar{\lambda}}-\frac{\bar{\lambda}}{2} \kappa_{\bar{\lambda}}+\bar{\varsigma} \kappa_{\bar{\lambda}}=-\left[\begin{array}{ll}
B_{\Psi}^{*} & 1
\end{array}\right] \Pi_{\bar{\lambda}}^{\Gamma}\left[\begin{array}{c}
y_{\bar{\lambda}}-B_{\Psi}^{\Gamma} \kappa_{\bar{\lambda}} \\
\kappa
\end{array}\right], \quad \kappa_{\bar{\lambda}}\left(s_{0}\right)=\kappa_{0},
\end{aligned}
$$

The procedure is analogous to the one in section 4 (cf. Rod15b]), so we will not repeat the details here. However, it is important to recall that since we need (in general) strong solutions to deal with the nonlinearity, it is important that the initial condition satisfies the compatibility condition $z\left(s_{0}\right)=z_{0} \in V+B_{\Psi} \mathbb{R}^{M}$ (cf.Lemma 7.4). This means that when dealing with stabilization to trajectories (cf. section 5, for the internal controls case), we need the compatibility condition

$$
\left.\left(y\left(s_{0}\right)-\hat{y}\left(s_{0}\right)\right)\right|_{\Gamma} \in B_{\Psi}^{\Gamma} \mathbb{R}^{M}=\operatorname{span}\left\{1_{\Gamma_{\mathrm{c}}} \chi_{\Gamma} \widehat{\Psi}_{i}\right\} .
$$


At this point we also recall that the solutions given in Lemmas 7.2 and 7.4 do not depend on the extension $E_{1}$ and $E_{2}$, respectively. See [Rod14, Remark 3.2].

Similar estimates on the transient bound, and on dimension of the control like (26) and (27), depending exponentially on the data, can also be derived.

It turns out that, in both internal and boundary cases, numerical simulations do suggest that a better estimate on the number of needed actuators might exist, like the one in (28) depending polynomially on the data. So, we would like to finish this section with two very particular questions:

- Can we find a particular case, in the boundary setting, leading to an estimate like (28)?

- How does the observability constant $C_{\text {obs }}$ in (60) "looks like" when the control acts on all the boundary? Can we get, in this case, a constant which is "better" than those in (61)?

Remark 7.14. Though the closed-loop systems $\{(73 \mathrm{a}),(73 \mathrm{c})\}$ and $(74)$ are formally equivalent, the latter may have some advantages for numerical simulations, because the dynamical equation in $(74 \mathrm{~b})$ is relatively easier to discretize than the integral equation in (73c). Hereafter, we will consider only the discretization of (74).

7.7. Back to original time. Stabilization to trajectories. Let us be give a solution $\hat{y}$ for the uncontrolled system (2) (with $u=0$ ) with $\hat{y}_{0}:=\hat{y}(0) \in H$ and such that the vector functions in (5) are such that $\widehat{\mathcal{N}}$ satisfies (44) and (45), and $(\hat{a}, \hat{b})$ satisfies (46), for suitable nonnegative constants $\widehat{C}$ and $C_{\mathcal{W} \text {,st }}$. Notice that, recalling the notation in section 2 , in this case $\mathcal{N}:=\frac{1}{\nu} \breve{\hat{\mathcal{N}}}$ also satisfies 44) and (45), and $(a, b)=\left(\frac{1}{\nu} \breve{\hat{a}}, \frac{1}{\nu} \hat{\hat{b}}\right)$ also satisfies (46),

We consider system (2) with a dynamical feedback as follows

$$
\begin{array}{rlrl}
\partial_{t} y-\nu \Delta y+f(y, \nabla y)=0, & \left.y\right|_{\Gamma} & =g-B_{\Psi}^{\Gamma} \kappa, \\
\partial_{t} \kappa+\varsigma \kappa=-\left[\begin{array}{ll}
B_{\Psi}^{*} & 1
\end{array}\right] \widehat{\Pi}_{\lambda}^{\Gamma}\left[\begin{array}{c}
y-\hat{y}-B_{\Psi}^{\Gamma} \kappa \\
\kappa
\end{array}\right], & y(0)=y_{0},
\end{array}
$$

with $\widehat{\Pi}_{\lambda}$ solving

$$
\frac{\mathrm{d}}{\mathrm{d} t} \widehat{\Pi}_{\lambda}^{\Gamma}+\widehat{\Pi}_{\lambda}^{\Gamma} \mathbb{A}_{\lambda, \varsigma}^{\hat{a}, \hat{b}, \nu}+\mathbb{A}_{\lambda, \varsigma}^{\hat{a}, \hat{b}, \nu} \widehat{\Pi}_{\lambda}^{\Gamma}-\widehat{\Pi}_{\lambda}^{\Gamma} B_{M}^{\Gamma} \mathcal{R}^{-1} B_{M}^{\Gamma} \widehat{\Pi}_{\lambda}^{\Gamma}+\mathcal{M}^{*} \mathcal{M}=0
$$

with $\mathcal{R}$ and $\mathcal{M}$ as in $(70)$ and with

$$
\mathbb{A}_{\lambda, \varsigma}^{\hat{a}, \hat{b}, \nu}=-\left[\begin{array}{cc}
-\nu \Delta+K^{\hat{a}, \hat{b}, \lambda} & K^{\hat{a}, \hat{b}, 0} \\
0 & \varsigma_{\lambda}
\end{array}\right],
$$

and $K^{\hat{a}, \hat{b}, r}$ and $\varsigma_{\lambda}$ defined as in $(69)$.

Observe that $(\breve{z}, \breve{\kappa})(\tau):=(y-\hat{y}, \kappa)\left(\frac{\tau}{\nu}\right)$ solves, with

$$
\begin{array}{rlrl}
\partial_{\tau} \breve{z}-\Delta \breve{z}+a \breve{z}+\nabla \cdot(b \breve{z}) & =\mathcal{N}(\breve{z}) ; & \left.\breve{z}\right|_{\Gamma} & =-B_{\Psi}^{\Gamma} \breve{\kappa}, \\
\partial_{t} \breve{\kappa}+\frac{\varsigma}{\nu} \breve{\kappa}=-\left[\begin{array}{ll}
B_{\Psi}^{*} & 1
\end{array}\right] \breve{\Pi}_{\lambda}^{\Gamma}\left[\begin{array}{c}
\breve{z}-B_{\Psi}^{\Gamma} \breve{\kappa} \\
\breve{\kappa}
\end{array}\right], & \breve{z}(0) & =y_{0}-\hat{y}_{0},
\end{array}
$$

and $\breve{\Pi}_{\lambda}^{\Gamma}(\tau)=\widehat{\Pi}_{\lambda}^{\Gamma}\left(\frac{\tau}{\nu}\right)$ solves $(70)$ with a different pair $(\mathcal{R}, \mathcal{M})$. Indeed, from $(77)$, we obtain that for $(\bar{\lambda}, \bar{\varsigma})=\left(\frac{\lambda}{\nu}, \frac{\varsigma}{\nu}\right)$,

$$
\frac{\mathrm{d}}{\mathrm{d} \tau} \breve{\widehat{\Pi}}_{\lambda}^{\Gamma}+\breve{\widehat{\Pi}}_{\lambda} \mathbb{A}_{\bar{\lambda}, \bar{\varsigma}}^{a, b, 1}+\mathbb{A}_{\bar{\lambda}, \bar{\varsigma}}^{a, b, 1^{*}} \breve{\widehat{\Pi}}_{\lambda}-\breve{\widehat{\Pi}}_{\lambda} B_{M}(\nu \mathcal{R})^{-1} B_{M}^{*} \breve{\widehat{\Pi}}_{\lambda}+\left(\left(\frac{1}{\nu}\right)^{\frac{1}{2}} \mathcal{M}\right)^{*}\left(\left(\frac{1}{\nu}\right)^{\frac{1}{2}} \mathcal{M}\right)=0 .
$$

Therefore, we can conclude that

$$
|\breve{z}(\tau)|_{V}^{2} \leq C \mathrm{e}^{-\bar{\lambda} \tau}|\breve{z}(0)|_{V}^{2}, \quad \text { for all } \tau \geq 0,
$$


provided $|\breve{z}(0)|_{V}$ is small enough. This implies that

$$
|y(t)-\hat{y}(t)|_{V}^{2} \leq C \mathrm{e}^{-\lambda t}\left|y_{0}-\hat{y}_{0}\right|_{V}^{2}, \quad \text { for all } t \geq 0,
$$

and we can conclude that the following theorem holds true. Recall the operators (63) and (67).

Theorem 7.15. Under the hypotheses of Proposition 7.12, with

$$
\operatorname{Ran} P_{M}=\operatorname{span}\left\{\widehat{\Psi}_{i} \mid i \in\{1,2, \ldots, M\}\right\} \subset H^{\frac{3}{2}}(\Gamma, \mathbb{R}),
$$

there exists $\epsilon>0$ with the following properties: if $y_{0} \in H$ is such that

$$
y_{0}-\hat{y}_{0} \in V+B_{\Psi} \mathbb{R}^{M} \quad \text { and } \quad\left|y_{0}-\hat{y}_{0}\right|_{V}<\epsilon,
$$

then the solution $y$ of the system (76) goes exponentially to $\hat{y}$ with rate $\frac{\lambda}{2}$, that is,

$$
|y(t)-\hat{y}(t)|_{V}^{2} \leq C \mathrm{e}^{-\lambda t}\left|y_{0}-\hat{y}_{0}\right|_{V}^{2}, \quad \text { for all } t \geq 0,
$$

for a suitable constant $C$ independent of $\left(\epsilon, y_{0}-\hat{y}_{0}\right)$. Furthermore, the solution $y$ is, and is unique, in the affine space $\hat{y}+L_{\text {loc }}^{2}\left(\mathbb{R}_{0}, \mathrm{D}(A)+B_{\Psi} \mathbb{R}^{M}\right) \cap C\left([0,+\infty), V+B_{\Psi} \mathbb{R}^{M}\right)$.

Notice that the feedback control stabilizes the linearized system to zero globally, that is, we have the following theorem.

Theorem 7.16. Under the hypotheses of Proposition 7.12, with

$$
\operatorname{Ran} P_{M}=\operatorname{span}\left\{\widehat{\Psi}_{i} \mid i \in\{1,2, \ldots, M\}\right\} \subset H^{\frac{3}{2}}(\Gamma, \mathbb{R}),
$$

given $\left(z_{0}, \kappa_{0}\right) \in H \times \mathbb{R}^{M}$, the solution of

$$
\begin{array}{rlrl}
\partial_{t} z-\nu \Delta z+a z+\nabla \cdot(b z)=0, & \left.z\right|_{\Gamma}=-B_{\Psi}^{\Gamma} \kappa, \\
\partial_{t} \kappa+\varsigma \kappa=-\left[\begin{array}{ll}
B_{\Psi}^{*} & 1
\end{array}\right] \widehat{\Pi}_{\lambda}^{\Gamma}\left[\begin{array}{c}
z-B_{\Psi}^{\Gamma} \kappa \\
\kappa
\end{array}\right], & {\left[\begin{array}{c}
z(0) \\
\kappa(0)
\end{array}\right]} & =\left[\begin{array}{c}
z_{0} \\
\kappa_{0}
\end{array}\right] .
\end{array}
$$

satisfies

$$
|z(t)|_{H}^{2} \leq C \mathrm{e}^{-\lambda t}\left|z_{0}\right|_{H}^{2}, \quad \text { for all } t \geq 0
$$

for a suitable constant $C$ independent of $z_{0}$. Furthermore, the solution $z$ is, and is unique, in the space $L_{\mathrm{loc}}^{2}\left(\mathbb{R}_{0}, \mathrm{D}(A)+B_{\Psi} \mathbb{R}^{M}\right) \cap C\left([0,+\infty), V+B_{\Psi} \mathbb{R}^{M}\right)$.

\section{DisCRETIZATION OF THE LineAR SYSTEMS}

We explain how we discretize a linear parabolic equation with nonhomogeneous Dirichlet boundary conditions. We will focus our simulations on the $2 \mathrm{D}$ case, considering our domain to be the unit ball $\Omega=\mathbb{D}:=\left\{\left(x_{1}, x_{2}\right) \in \mathbb{R}^{2} \mid x_{1}^{2}+x_{2}^{2}<1\right\}$.

Here, we focus on the approximation of the linearized closed-loop systems (56) and $(79)$ perturbed with the reaction term $\frac{\lambda}{2} z$ :

$$
\begin{aligned}
\partial_{t} z-\nu \Delta z+\left(\hat{a}-\frac{\lambda}{2}\right) z+\nabla \cdot(\hat{b} z)+\mathcal{F}_{\lambda}^{\text {in }} z & =0,\left.\quad z\right|_{\Gamma}=0, \\
z(0) & =z_{0},
\end{aligned}
$$

and

$$
\begin{aligned}
\partial_{t} z-\nu \Delta z+\left(\hat{a}-\frac{\lambda}{2}\right) z+\nabla \cdot(\hat{b} z) & =0, & \left.z\right|_{\Gamma} & =B_{\Psi}^{\Gamma} \kappa, \\
\partial_{t} \kappa+\left(\varsigma-\frac{\lambda}{2}\right) \kappa+\mathcal{F}_{\lambda}^{\mathrm{bo}}\left(z-B_{\Psi} \kappa, \kappa\right) & =0, & (z(0), \kappa(0)) & =\left(z_{0}, \kappa_{0}\right),
\end{aligned}
$$

with $\mathcal{F}_{\lambda}^{\text {in }} z:=B_{M} \mathcal{R}^{-1} B_{M}^{*} \widehat{\Pi}_{\lambda} z$ and $\mathcal{F}_{\lambda}^{\text {bo }}\left(z-B_{\Psi} \kappa, \kappa\right):=\left[\begin{array}{ll}B_{\Psi}^{*} & 1\end{array}\right] \widehat{\Pi}_{\lambda}^{\Gamma}\left[\begin{array}{c}z-B_{\Psi} \kappa \\ \kappa\end{array}\right]$. 
Remark 8.1. Notice that we want to observe that the solutions of (56), respectively (79) go to zero exponentially with rate $\frac{\lambda}{2}$. Thus we want the solutions of (80) and 81) to remain bounded. Indeed $z$ solves $(\sqrt[56)]{ }$, respectively $(z, \kappa)$ solves $(79)$, if and only if $\mathrm{e}^{\frac{\lambda}{2}} \cdot z$ solves (80), respectively $\mathrm{e}^{\frac{\lambda}{2}} \cdot(z, \kappa)$ solves 81 .

8.1. Discretization in space. The simulations are done in MATLAB. We approximate $\Omega$ by a polygonal domain $\Omega_{D}$ and consider a partition of $\Omega_{D}$ into nonoverlapping triangles. For this we use the function initmesh from MATLAB. This function gives us a mesh triple $(\mathbf{p}, \mathbf{e}, \mathbf{t})$ where

- the point matrix $\mathbf{p}$ contains (information about) all the vertices of all triangles of the partition.

- the edge matrix e contains (information about) all the boundary segments of $\Omega_{D}$.

- the triangle matrix $\mathbf{t}$ contains (information about) the triangles of the partition.

Recall that given a triangle $\mathbf{t}_{k}$ with vertices $\left(\mathbf{p}_{\mathbf{t}_{k}(1)}, \mathbf{p}_{\mathbf{t}_{k}(2)}, \mathbf{p}_{\mathbf{t}_{k}(3)}\right)$, then any point $x \in$ $\mathbf{t}_{k}$ (inside or on the boundary of the triangle $\mathbf{t}_{k}$ ) can be written uniquely as a convex combination: there are nonnegative $x^{\mathbf{t}_{k}(1)}, x^{\mathbf{t}_{k}(2)}$, and $x^{\mathbf{t}_{k}(3)}$ such that

$$
\begin{aligned}
& 1=x^{\mathbf{t}_{k}(1)}+x^{\mathbf{t}_{k}(2)}+x^{\mathbf{t}_{k}(3)}, \\
& x=x^{\mathbf{t}_{k}(1)} \mathbf{p}_{\mathbf{t}_{k}(1)}+x^{\mathbf{t}_{k}(2)} \mathbf{p}_{\mathbf{t}_{k}(2)}+x^{\mathbf{t}_{k}(3)} \mathbf{p}_{\mathbf{t}_{k}(3)} .
\end{aligned}
$$

Any continuous function $z \in C(\bar{\Omega}, \mathbb{R})$ can, and will, be approximated by a sum

$$
z(x) \approx \tilde{z}(x):=\sum_{i=1}^{s_{\mathbf{p}}} z\left(\mathbf{p}_{i}\right) \widehat{\phi}_{i}(x), \quad \text { for all } \quad x \in \overline{\Omega_{D}},
$$

where $s_{\mathbf{p}}$ is the total number of points in the mesh; $\left\{\mathbf{p}_{i} \mid i=1,2, \ldots, s_{\mathbf{p}}\right\}$ is the set of all mesh points and the $\widehat{\phi}_{i}$ s are the classical piecewise linear hat functions defined as

$$
\widehat{\phi}_{i}(x):= \begin{cases}1, & \text { if } x=\mathbf{p}_{i} ; \\ 0, & \text { if } x=\mathbf{p}_{j} \text { with } j \neq i ; \\ \sum_{l=1}^{3} x^{\mathbf{t}_{k}(l)} \widehat{\phi}_{i}\left(\mathbf{p}_{\mathbf{t}_{k}(l)}\right), & \text { if } x \in \mathbf{t}_{k} .\end{cases}
$$

Notice that the support of $\phi_{i}$ consists of the triangles with the common vertex $\mathbf{p}_{i}$. We will denote the finite-dimensional space

$$
V_{D}:=\operatorname{span}\left\{\widehat{\phi}_{i} \mid i \in\left\{1,2, \ldots, s_{\mathbf{p}}\right\}\right\} .
$$

In other words the function $z$ can be approximated by the evaluation vector

$$
\bar{z}:=\left[\begin{array}{llll}
z\left(\mathbf{p}_{1}\right) & z\left(\mathbf{p}_{2}\right) & \ldots & z\left(\mathbf{p}_{s_{\mathbf{p}}}\right)
\end{array}\right]^{\top} \in \mathcal{M}_{s_{\mathbf{p}} \times 1}
$$

where $A^{\top}$ stands for the transpose matrix of $A$.

The weak discretization matrix $\mathbf{L}_{D}$ of a given operator $L \in \mathcal{L}\left(H^{1} \rightarrow V^{\prime}\right)$, is defined so that (for smooth functions)

$$
\bar{v}^{\top} \mathbf{L}_{D} \bar{u}:=(L \tilde{u}, \tilde{v})_{H}=(\tilde{v}, L \tilde{u})_{H},
$$

that is, the entry in the $i$ th row and $j$ th column of $\mathbf{L}_{D}$ is $\left(\mathbf{L}_{D}\right)_{(i, j)}=\left(\widehat{\phi}_{i}, L \widehat{\phi}_{j}\right)_{H}$.

We recall the so called mass $\mathbf{M}$ and stiffness $\mathbf{S}$ matrices are defined as

$$
\mathbf{M}_{(i, j)}=\left(\widehat{\phi}_{i}, \widehat{\phi}_{j}\right)_{H} \quad \text { and } \quad \mathbf{S}_{(i, j)}:=\left(\nabla \widehat{\phi}_{i}, \nabla \widehat{\phi}_{j}\right)_{H}
$$

Notice that $\mathbf{M}=(I d)_{D}$ where $I d: v \mapsto v$ is the identity/inclusion operator, and that $\mathbf{S}$ is related to the Laplacian: for smooth $(u, v) \in H^{1}(\Omega) \times H_{0}^{1}(\Omega)$ we have $\langle-\Delta u, v\rangle_{V^{\prime}, V}=$ $(\nabla u, \nabla v)_{H} \approx(\nabla \tilde{u}, \nabla \tilde{v})_{H}=\bar{v}^{\top} \mathbf{S} \bar{u}$.

We refer to [Che05, Section 1.3 to 1.5] for further details. 
8.1.1. Discretization of the heat equation. We are ready to semi-discretize the system

$$
\begin{aligned}
\partial_{t} z-\nu \Delta z+f & =0, \\
\left.z\right|_{\Gamma}=g, \quad z(0) & =z_{0},
\end{aligned}
$$

provided the functions $f$ and $g$ are known (and continuous in space variable).

Inspired from Lemma 7.2 we look for a solution in $W\left(I, H^{1}, V^{\prime}\right)$, thus we look at $82 \mathrm{a}$ ) as an identity in $L^{2}\left(I, V^{\prime}\right)$. Therefore, it is enough to test this equation with elements $w$ in the dual $L^{2}(I, V)$. Replacing $z$ by $\tilde{z}$ and testing with a function $\tilde{w} \in C\left(I, V_{D}\right)$ with $\left.\tilde{w}\right|_{\Gamma}=0$, we find that $\tilde{z} \in L^{2}(I, V)$ and $(\Delta \tilde{z}, \tilde{w})=-\bar{w}^{\top} \mathbf{S} \bar{z}$, and we arrive to

$$
\bar{w}^{\top} \partial_{t} \mathbf{M} \bar{z}+\nu \bar{w}^{\top} \mathbf{S} \bar{z}+\bar{w}^{\top} \mathbf{M} \bar{f}=0,\left.\quad \bar{z}\right|_{\Gamma}=\bar{g} .
$$

Reordering the mesh points. The vector $\mathbf{p}$ contains both interior and boundary points of $\Omega$ (or $\Omega_{D}$ ). We define the permutation matrix $\mathbf{P}^{\mathbf{i b}}$ such that the boundary points will appear at the end of the vector (and the relative order of interior (resp. boundary) points is unchanged). We can find which indices correspond to the boundary points from the information we have in the edge vector $\mathbf{e}$. In the new coordinates $\bar{z}_{*}=\mathbf{P}$ $\bar{z}_{*}=:\left[\begin{array}{l}\bar{z}_{* \mathrm{i}} \\ \bar{z}_{* \mathrm{~b}}\end{array}\right]$ where the vector $\bar{z}_{* \mathrm{i}}$ correspond to the values at the interior points and $\bar{z}_{* \mathrm{~b}}$ to the values at the boundary points. Thus we arrive at

$$
\bar{w}_{*}^{\top} \partial_{t} \mathbf{M}_{*} \bar{z}_{*}+\nu w_{*}^{\top} \mathbf{S}_{*} \bar{z}_{*}^{\top}+\bar{w}_{*}^{\top} \mathbf{M}_{*} \bar{f}_{*}=0, \quad \bar{z}_{* \mathrm{~b}}=\bar{g}
$$

with $\mathbf{M}_{*}=\mathbf{P}^{\mathbf{i b}} \mathbf{M} \mathbf{P}^{\mathbf{i b} \top}$ and $\mathbf{S}_{*}=\mathbf{P}^{\mathbf{i b}} \mathbf{S} \mathbf{P}^{\mathbf{i b} \top}$. Notice that the inverse and transpose of a permutation matrix do coincide. Writing $\mathbf{M}_{*}$ and $\mathbf{S}_{*}$ in blocks notation

$$
\mathbf{M}_{*}=:\left[\begin{array}{ll}
\mathbf{M}_{* \mathrm{ii}} & \mathbf{M}_{* \mathrm{bb}} \\
\mathbf{M}_{* \mathrm{bi}} & \mathbf{M}_{* \mathrm{bb}}
\end{array}\right] \text { and } \mathbf{S}_{*}=:\left[\begin{array}{cc}
\mathbf{S}_{* \mathrm{ii}} & \mathbf{S}_{* \mathrm{ib}} \\
\mathbf{S}_{* \mathrm{bi}} & \mathbf{S}_{* \mathrm{bb}}
\end{array}\right]
$$

and recalling that $\bar{w}_{* \mathrm{~b}}^{\top}=0$ we obtain

$$
\bar{w}_{* \mathrm{i}}^{\top} \partial_{t}\left[\begin{array}{ll}
\mathbf{M}_{* \mathrm{ii}} & \mathbf{M}_{* \mathrm{ib}}
\end{array}\right]\left[\begin{array}{c}
\bar{z}_{* \mathrm{i}} \\
\bar{z}_{* \mathrm{~b}}
\end{array}\right]+\nu \bar{w}_{* \mathrm{i}}^{\top}\left[\begin{array}{ll}
\mathbf{S}_{* \mathrm{ii}} & \mathbf{S}_{* \mathrm{ib}}
\end{array}\right]\left[\begin{array}{c}
\bar{z}_{* \mathrm{i}} \\
\bar{z}_{* \mathrm{~b}}
\end{array}\right]+\bar{w}_{* \mathrm{i}}^{\top}\left[\begin{array}{ll}
\mathbf{M}_{* \mathrm{ii}} & \mathbf{M}_{* \mathrm{ib}}
\end{array}\right]\left[\begin{array}{c}
\bar{f}_{* \mathrm{i}} \\
\bar{f}_{* \mathrm{~b}}
\end{array}\right]=0 .
$$

Remark 8.2. In what follows we will work in the new coordinates and for simplicity we will skip the subscript $*$.

Therefore, taking into account Remark 8.2, we arrive to the semi-discrete system

$$
\partial_{t} \mathbf{M}_{\mathrm{ii}} \bar{z}_{\mathrm{i}}=-\nu \mathbf{S}_{\mathrm{ii}} \bar{z}_{\mathrm{i}}-\nu \mathbf{S}_{\mathrm{ib}} \bar{z}_{\mathrm{b}}-\partial_{t} \mathbf{M}_{\mathrm{ib}} \bar{z}_{\mathrm{b}}-\left[\begin{array}{ll}
\mathbf{M}_{\mathrm{ii}} & \mathbf{M}_{\mathrm{ib}}
\end{array}\right] \bar{f}
$$

which underlines that when $\bar{g}$ (i.e., $\bar{z}_{\mathrm{b}}$ ) is known then the number of unknowns (i.e., the entries of $\left.\bar{z}_{\mathbf{i}}\right)$ is reduced to the number of interior points $s_{\mathbf{p}}-s_{\mathbf{e}}$, where $s_{\mathbf{e}}$ is the total number of points in the boundary.

Remark 8.3. In the elliptic case, $\partial_{t} \mathbf{M} \bar{z}=0$, we see that $(83)$ reduces to $\nu \mathbf{S}_{\mathrm{ii}} \bar{z}_{\mathrm{i}}=-\nu \mathbf{S}_{\mathrm{ib}} \bar{z}_{\mathrm{b}}-$ $\left[\begin{array}{ll}\mathbf{M}_{\mathrm{ii}} & \mathbf{M}_{\mathrm{ib}}\end{array}\right] \bar{f}$ which are the system we find in [FGP83, section 2] when $\bar{z}_{\mathrm{b}}$ is given. See also [FGP83, section 1] for references to other methods to deal with nonhomogeneous boundary conditions.

8.1.2. Discretization of a composition of linear operators. In order to discretize systems (80) and (81) we follow a simple idea. See, for example, [KR15b, Section 5.1].

Given two operators $L_{1} \in \mathcal{L}\left(H^{1}, Z\right)$ and $L_{2} \in \mathcal{L}\left(Z, V^{\prime}\right)$, where $Z \subset H^{1}$ is an Hilbert space then the composition $L_{2} \circ L_{1}$ is in $\mathcal{L}\left(H^{1}, V^{\prime}\right)$. Suppose we know the discretization matrix $\left(L_{2}\right)_{D}$ and also an evaluation matrix $\overline{L_{1}}$ which approximates $L_{1}$, that is, $\overline{L_{1}} \bar{v} \approx \overline{L_{1} v}$. Then we may write

$$
\bar{w}^{\top}\left(L_{2} \circ L_{1}\right)_{D} \bar{z} \approx\left(L_{2} \circ L_{1} z, w\right)_{H} \approx \bar{w}^{\top}\left(L_{2}\right)_{D} \overline{L_{1}} \bar{z} .
$$


Analogously if we know the discretization matrix $\left(L_{1}\right)_{D}$ and an evaluation matrix $\overline{L_{2}^{*}}$ of the adjoint $L_{2}^{*}$, we may write

$$
\bar{w}^{\top}\left(L_{2} \circ L_{1}\right)_{D} \bar{z} \approx\left(L_{2} \circ L_{1} z, w\right)_{H} \approx\left(\overline{\left(L_{2}\right)^{*}} \bar{w}\right)^{\top}\left(L_{1}\right)_{D} \bar{z}
$$

Therefore we have two candidates to approximate $\left(L_{2} \circ L_{1}\right)_{D}$ :

$$
\left(L_{2}\right)_{D} \overline{L_{1}} \approx\left(L_{2} \circ L_{1}\right)_{D} \approx{\overline{\left(L_{2}\right)^{*}}}^{\top}\left(L_{1}\right)_{D}
$$

Further notice that $\left(L^{*}\right)_{D}=L_{D}^{\top}$ and that

$$
{\overline{L^{*}}}^{\top} \mathbf{M} \approx L_{D} \approx \mathbf{M} \bar{L}, \quad \bar{L} \approx \mathbf{M}^{-1} L_{D}, \quad{\overline{L^{*}}}^{\top} \approx L_{D} \mathbf{M}^{-1}
$$

8.1.3. Discretization of the linear reaction and linear convection operators. We can construct the discretization matrices $\mathbf{G}_{x_{k}}:=\left(\partial_{x_{k}}\right)_{D},\left(\mathbf{G}_{x_{k}}\right)_{i j}:=\left(\phi_{i}, \partial_{x_{k}} \phi_{j}\right)_{H}$, of the directional derivatives operators, $k \in\{1,2\}$, in the same way as we construct the mass and stiffness matrices. Then for a function $w$, we set $\bar{w} \cdot:=\mathcal{D}_{\bar{w}}$, where $\mathcal{D}_{v}$ stands for the diagonal matrix whose diagonal is $v$. Observe that $2 w \cdot=\iota_{V} \circ(w \cdot)+(w \cdot) \circ \iota_{V}$. Finally for the linear reaction and convection operators, we take

$$
\left(\left(\hat{a}-\frac{\lambda}{2}\right) \cdot\right)_{D} \approx \frac{1}{2}\left(\mathbf{M} \mathcal{D}_{\bar{a}-\frac{\lambda}{2}}+\mathcal{D} \overline{\hat{a}-\frac{\lambda}{2}} \mathbf{M}\right) \quad \text { and } \quad(\nabla \cdot(\hat{b} \cdot))_{D} \approx \mathbf{G}_{x_{1}} \mathcal{D}_{\overline{\hat{b}}_{1}}+\mathbf{G}_{x_{2}} \mathcal{D}_{\overline{\hat{b}}_{2}}
$$

Notice that $w \cdot=(w \cdot)^{*}$ and by taking the semi-sum above as an approximation for $(w \cdot)_{D}$ we preserve the symmetry. We must say, however that in our simulations we did not observe much difference when we have simply taken $(w \cdot)_{D} \approx \mathbf{M} \mathcal{D}_{\bar{w}}$.

Notice also that the above discretization idea would lead us to take $(\hat{b} \cdot \nabla \cdot) \approx \mathcal{D}_{\bar{b}_{1}} \mathbf{G}_{x_{1}}+$ $\mathcal{D}_{\bar{b}_{2}} \mathbf{G}_{x_{2}}$, and that looking at the operators as $\mathcal{L}\left(H^{1}, V^{\prime}\right)$, we need to test the operators with functions/vectors vanishing at the boundary. Now, for either $\left.v\right|_{\Gamma}=0$ or $\left.w\right|_{\Gamma}=0$, the definition of $\mathbf{G}_{x_{k}}$, gives us $\left(\tilde{v}, \partial_{x_{k}} \tilde{w}\right)_{H}=-\left(\partial_{x_{k}} \tilde{v}, \tilde{w}\right)_{H}$, that is, $\bar{v}^{\top} \mathbf{G}_{x_{k}} \bar{w}=-\bar{w}^{\top} \mathbf{G}_{x_{k}} \bar{v}$. Now our approximations give $\bar{v}^{\top}\left(\mathbf{G}_{x_{1}} \mathcal{D}_{\bar{b}_{1}}+\mathbf{G}_{x_{2}} \mathcal{D}_{\bar{b}_{2}}\right) \bar{w}=\bar{w}^{\top}\left(\mathcal{D}_{\bar{b}_{1}} \mathbf{G}_{x_{1}}^{\top}+\mathcal{D}_{\overline{\hat{b}}_{2}} \mathbf{G}_{x_{2}}^{\top}\right) \bar{v}=$ $\bar{w}^{\top}\left(-\mathcal{D}_{\bar{b}_{1}} \mathbf{G}_{x_{1}}-\mathcal{D}_{\overline{\hat{b}}_{2}} \mathbf{G}_{x_{2}}\right) \bar{v}$. Therefore, $\left(\mathbf{G}_{x_{1}} \mathcal{D}_{\bar{b}_{1}}+\mathbf{G}_{x_{2}} \mathcal{D}_{\overline{\hat{b}}_{2}}\right)^{\top}=-\mathcal{D}_{\overline{\hat{b}}_{1}} \mathbf{G}_{x_{1}}-\mathcal{D}_{\bar{b}_{2}} \mathbf{G}_{x_{2}}$, which agrees with the fact that we have $(w, \nabla \cdot(\hat{b} v))_{H}=(-\hat{b} \cdot \nabla w, v)_{H}$.

Remark 8.4. In some situations, for example when the viscosity coefficient is small and the exact solution is known to have some big magnitude directional space derivatives, it may be the case that the numerical solution present some oscillations. For solving such problems with a reasonable number of mesh points, avoiding or minimizing the oscillations, we may need more sophisticated numerical discretizations. We refer to [JS08] and to [JK07, JK08] for such discretizations.

Remark 8.5. Below, we will consider the stabilization to a trajectory $\hat{y}$ (given a priori) whose first order derivatives are not too big, which is also motivated from the discussion in Section 5. Therefore, remaining close to $\hat{y}$ the solutions will not present big first order derivatives and we may expect that no big oscillations will be observed on the numerical solution. From the control point of view, if some small oscillations do appear, then we could thing of them as small perturbations of the solution and, since the control will be given in feedback form, we may expect the control to be able to respond to such perturbations. Roughly speaking, we could see those small perturbations as a further test for the robustness of the control. 
8.1.4. Discretization of the feedback control. We start by recalling that the feedback depends on the solution $\Pi$ of the corresponding Riccati equation and is self-adjoint, that is, we know that $\Pi_{D}$ is symmetric. We look for an approximation of $\Pi_{D}$ by solving a suitable matrix Riccati equation

$$
\partial_{t} \Pi_{D}+\Pi_{D} \mathbf{X}+\mathbf{X}^{\top} \Pi_{D}-\Pi_{D} \mathbf{R} \mathbf{R}^{\top} \Pi_{D}+\mathbf{C}^{\top} \mathbf{C}=0, \quad t>0,
$$

For $r \geq 0$, let us introduce the matrices

$$
\mathbf{K}_{\mathbf{r}}(t):=\mathbf{M}^{-1}\left(\mathbf{G}_{x_{1}} \mathcal{D}_{\overline{\hat{b}_{1}(t)}}+\mathbf{G}_{x_{2}} \mathcal{D}_{\overline{\hat{b}_{2}(t)}}\right)+\mathcal{D}_{\overline{\hat{a}-\frac{r}{2}(t)}}=:\left[\begin{array}{cc}
\mathbf{K}_{r, \mathrm{ii}}(t) & \mathbf{K}_{r, \mathrm{ib}}(t) \\
\mathbf{K}_{r, \mathrm{bi}}(t) & \mathbf{K}_{r, \mathrm{bb}}(t)
\end{array}\right] .
$$

Internal feedback. For system (80) we need to solve (55). Since $\left.z\right|_{\Gamma}=0$, we look for matrices in $\mathcal{M}_{\left(s_{\mathbf{p}}-s_{\mathbf{e}}\right) \times\left(s_{\mathbf{p}}-s_{\mathbf{e}}\right)}$.

We choose the matrices $\mathbf{C}^{\mathrm{in}}=\nu^{\frac{1}{2}} \mathbf{S}_{\mathrm{ii}, \mathrm{c}}$ and $\mathbf{X}_{\lambda}^{\mathrm{in}}=\mathbf{X}_{\lambda}^{\mathrm{in}}(t)=-\nu \mathbf{M}_{\mathrm{ii}}^{-1} \mathbf{S}_{\mathrm{ii}}-\mathbf{K}_{\lambda, \mathrm{ii}}(t)$, where $\mathbf{S}_{\mathrm{ii}, \mathrm{c}}$ is the Cholesky factor of $\mathbf{S}_{\mathrm{ii}}$, which gives us $\left(\mathbf{C}^{\mathrm{in}}\right)^{\top}\left(\mathbf{C}^{\mathrm{in}}\right)=\nu \mathbf{S}_{\mathrm{ii}}$. To construct the matrix $\mathbf{R}^{\text {in }}$ we set $\mathcal{R}=1$ in (55) and observe that we must have

$$
\Pi_{D} \mathbf{R}^{\mathrm{in}} \mathbf{R}^{\mathrm{in} \top} \Pi_{D} \approx\left(\Pi 1_{\omega} \chi P_{M} 1_{\omega} \mathcal{R} 1_{\omega} P_{M} \chi 1_{\omega} \Pi\right)_{D}
$$

We will also take actuators $\left\{\overline{\Phi_{i}} \mid i \in 1,2, \ldots, M\right\}$ which are supported in $\bar{\omega}$, thus since $P_{M}$ stands for the orthogonal projection onto $\operatorname{span}\left\{\overline{\Phi_{i}} \mid i \in 1,2, \ldots, M\right\}$, we may write

$$
\left(\Pi 1_{\omega} \chi P_{M} 1_{\omega} \mathcal{R} 1_{\omega} P_{M} \chi 1_{\omega} \Pi\right)_{D} \approx \Pi_{D} \overline{1_{\omega} \chi P_{M} \chi 1_{\omega} \Pi}
$$

and observe that the natural evaluation matrix of the multiplication $\overline{1_{\omega} \chi} \cdot=\overline{\chi_{\omega}}$. is just the diagonal matrix $\mathcal{D} \overline{\chi_{1} 1_{\omega}}$ when we identify $\chi 1_{\omega}$ with the function that takes the value 1 if $x \in \omega \cap \operatorname{supp} \chi$ and the value 0 otherwise. To construct an evaluation matrix of the $H$-orthogonal projection $P_{M}$, we start by orthonormalizing, in the $\mathbf{M}_{\mathrm{ii}}$-scalar product, the family of actuators $\left\{\bar{\Phi}_{i \mathrm{i}} \mid i \in 1,2, \ldots, M\right\}$. Then we denote the orthonormal family by $\left\{\bar{\Phi}_{i}^{o} \mid i \in 1,2, \ldots, M\right\}$ and set

$$
\overline{P_{M}}=\mathbf{P}_{M}:=S_{M} S_{M}^{\top} \mathbf{M}_{\mathrm{ii}}, \quad \text { with } \quad S_{M}:=\left[\begin{array}{llll}
{\overline{\Phi_{1}}}^{o} & {\overline{\Phi_{2}}}^{o} & \ldots & {\overline{\Phi_{M}}}^{o}
\end{array}\right],
$$

which leads us to

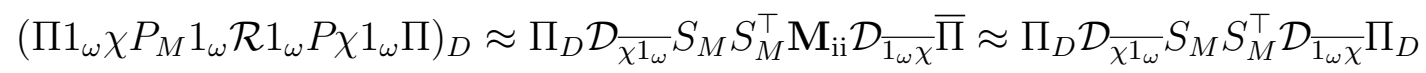

and this is the reason we propose to take the matrix

$$
\mathbf{R}^{\text {in }}:=\mathcal{D} \frac{\overline{\chi 1_{\omega}}}{S_{M}} .
$$

Therefore, as the approximation evaluation matrix of the feedback control rule we set

$$
\overline{\mathcal{F}_{\lambda}^{\text {in }}}:=\left(\mathbf{R}^{\text {in }}\right)\left(\mathbf{R}^{\text {in }}\right)^{\top} \Pi_{D}
$$

We refer to [KR15b, section 5.3.3] for a different choice of $\mathbf{R}^{\text {in }}$, namely the less simple expression $\left(\mathbf{M}_{\mathrm{ii}, \mathrm{c}} \mathbf{P}_{M} \mathcal{D} \overline{\overline{\chi 1_{\omega}}} \mathbf{M}_{\mathrm{ii}}^{-1}\right)^{\top}$.

Boundary feedback. For system (81) we need to solve (77). Notice that (70) is related with the extended system (66) whose state belongs to $V \times \mathbb{R}^{M}$, thus we look for matrices in $\mathcal{M}_{\left(s_{\mathbf{p}}-s_{\mathbf{e}}+M\right) \times\left(s_{\mathbf{p}}-s_{\mathbf{e}}+M\right)}$.

First of all we given the boundary actuators $\Phi$ we construct the extensions $\widetilde{\Phi}$, defined in $\Omega$, by solving (numerically) the elliptic system

$$
-\nu \Delta \widetilde{\Psi}+\varsigma \widetilde{\Psi}_{i}=0,\left.\quad \widetilde{\Psi}_{i}\right|_{\Gamma}=\Psi_{i} .
$$

(which is equivalent to (68), with $\bar{\varsigma}=\frac{\varsigma}{\nu}$ ). 
We choose the matrices $\mathbf{C}^{\text {bo }}$ and $\mathbf{X}_{\lambda}^{\text {bo }}=\mathbf{X}_{\lambda}^{\text {bo }}(t)$ as

$$
\mathbf{C}^{\mathrm{bo}}=\left[\begin{array}{cc}
\nu^{\frac{1}{2}} \mathbf{S}_{\mathrm{ii}, \mathrm{c}} & 0 \\
0 & \mathbf{I}_{M}
\end{array}\right] \quad \text { and } \quad \mathbf{X}^{\mathrm{bo}}=\left[\begin{array}{cc}
\mathbf{X}_{\lambda}^{\mathrm{in}} & -\mathbf{K}_{0, \mathrm{ii}}\left[B_{\widetilde{\Psi}}\right]_{\mathrm{i}} \\
0 & -\varsigma_{M}
\end{array}\right],
$$

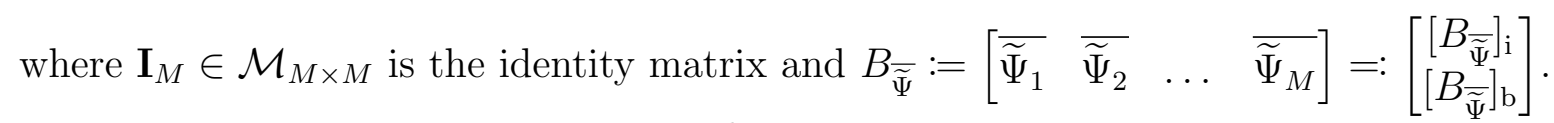

To choose an appropriate operator $\mathbf{R}^{\text {bo }}$ we start again by setting $\mathcal{R}=1$ and, looking at the nonlinear term of the Riccati equation we can formally write

$$
\left(\Pi\left[\begin{array}{l}
B_{\Psi} \\
\mathbf{I}_{M}
\end{array}\right]\left[\begin{array}{ll}
B_{\Psi}^{*} & \mathbf{I}_{M}
\end{array}\right] \Pi\right)_{D} \approx \Pi_{D}\left[\begin{array}{l}
B_{\Psi} \\
\mathbf{I}_{M}
\end{array}\right]\left[\begin{array}{ll}
\overline{B_{\Psi}^{*}} & \mathbf{I}_{M}
\end{array}\right] \bar{\Pi}
$$

From $\left(B_{\Psi} \kappa, y\right)_{H}=\sum_{j=1}^{M} \kappa_{j}\left(\widetilde{\Psi}_{j}, y\right)_{H}$ we may also set $\overline{B_{\Psi}^{*}}={\overline{B_{\Psi}}}^{\top} \mathbf{M}=B_{\widetilde{\Psi}}^{\top} \mathbf{M}$. For given $y \in V$ and $j \in\{1,2, \ldots, M\}$ we obtain

$$
\begin{aligned}
& \left(\widetilde{\Psi}_{j}, y\right)_{H} \approx \overline{\widetilde{\Psi}}_{j}^{\top} \mathbf{M} \bar{y}=\overline{\widetilde{\Psi}}_{j}^{\top}\left[\begin{array}{ll}
\mathbf{M}_{\mathrm{ii}} & \mathbf{M}_{\mathrm{bi}}
\end{array}\right] \bar{y}_{\mathrm{i}}=\left(\overline{\widetilde{\Psi}}_{j_{\mathrm{i}}}^{\top}+\overline{\widetilde{\Psi}}_{j_{\mathrm{b}}}^{\top} \mathbf{M}_{\mathrm{bi}} \mathbf{M}_{\mathrm{ii}}^{-1}\right) \mathbf{M}_{\mathrm{ii}} \bar{y}_{\mathrm{i}} \\
& =\left(\widetilde{\widetilde{\Psi}}_{j_{\mathrm{i}}}+\mathbf{M}_{\mathrm{ii}}^{-1} \mathbf{M}_{\mathrm{ib}} \widetilde{\widetilde{\Psi}}_{j_{\mathrm{b}}}\right)^{\top} \mathbf{M}_{\mathrm{ii}} \bar{y}_{\mathrm{i}} .
\end{aligned}
$$

and this is why, looking at $B_{\Psi}$ as an operator in $\mathcal{L}\left(\mathbb{R}^{M}, V^{\prime}\right)$, we take

$$
\overline{B_{\Psi}}=\left[\begin{array}{llll}
\bar{B}_{\Psi(1,1)} & \bar{B}_{\Psi(1,2)} & \ldots & \bar{B}_{\Psi(1, M)}
\end{array}\right] \quad \text { and } \quad{\overline{B_{\Psi}^{*}}}^{*}{\overline{B_{\Psi}}}^{\top} \mathbf{M}_{\mathrm{ii}},
$$

with $\overline{B_{\Psi}(1, j)}:=\overline{\widetilde{\Psi}}_{j_{\mathrm{i}}}+\mathbf{M}_{\mathrm{ii}}^{-1} \mathbf{M}_{\mathrm{ib}} \widetilde{\widetilde{\Psi}}_{j_{\mathrm{b}}}$.

Therefore, we arrive to

$$
\left(\Pi\left[\begin{array}{l}
B_{\Psi} \\
\mathbf{I}_{M}
\end{array}\right]\left[\begin{array}{ll}
B_{\Psi}^{\top} & \mathbf{I}_{M}
\end{array}\right] \Pi\right)_{D} \approx \Pi_{D}\left[\begin{array}{l}
B_{\Psi} \\
\mathbf{I}_{M}
\end{array}\right]\left[\begin{array}{ll}
\bar{B}_{\Psi}^{\top} & \mathbf{I}_{M}
\end{array}\right]\left[\begin{array}{cc}
\mathbf{M}_{\mathrm{ii}} & 0 \\
0 & \mathbf{I}_{M}
\end{array}\right] \bar{\Pi} .
$$

Since $\left[\begin{array}{cc}\mathbf{M}_{\mathrm{ii}} & 0 \\ 0 & \mathbf{I}_{M}\end{array}\right] \bar{\Pi} \approx \Pi_{D}$ we propose to take the matrix

$$
\mathbf{R}^{\text {bo }}:=\left[\begin{array}{l}
\overline{B_{\Psi}} \\
\mathbf{I}_{M}
\end{array}\right]
$$

and, as the approximation evaluation matrix of the feedback control rule, we set

$$
\overline{\mathcal{F}_{\lambda}^{\mathrm{bo}}}:=\left(\mathbf{R}^{\mathrm{bo}}\right)\left(\mathbf{R}^{\mathrm{bo}}\right)^{\top} \Pi_{D} .
$$

8.1.5. The semidicrete systems. We are now ready to present the semidiscrete versions of the linear closed-loop systems. Introducing the matrices

$$
\mathbf{L}_{\lambda}=\mathbf{L}_{\left(\hat{a}-\frac{\lambda}{2}, \hat{b}\right)}:=\frac{1}{2}\left(\mathbf{M} \mathcal{D}_{\bar{a}-\frac{\lambda}{2}}+\mathcal{D} \frac{\bar{a}-\frac{\lambda}{2}}{\mathbf{M}}\right)+\mathbf{G}_{x_{1}} \mathcal{D}_{\bar{b}_{1}}+\mathbf{G}_{x_{2}} \mathcal{D}_{\bar{b}_{2}}, \quad \mathbf{L}_{\lambda}=:\left[\begin{array}{cc}
\mathbf{L}_{\lambda, \mathrm{ii}} & \mathbf{L}_{\lambda, \mathrm{ib}} \\
\mathbf{L}_{\lambda, \mathrm{bi}} & \mathbf{L}_{\lambda, \mathrm{bb}}
\end{array}\right],
$$

system 80 is approximated by

$$
\partial_{t} \mathbf{M}_{\mathrm{ii}} \bar{z}_{\mathrm{i}}=-\nu \mathbf{S}_{\mathrm{ii}} \bar{z}_{\mathrm{i}}-\mathbf{L}_{\lambda, \mathrm{ii}} \bar{z}_{\mathrm{i}}-\mathbf{M}_{\mathrm{ii}} \overline{\mathcal{F}_{\lambda}^{\mathrm{in}}} \bar{z}_{\mathrm{i}},
$$

and system (81), setting $\varsigma_{\lambda}=\varsigma-\frac{\lambda}{2}$, is approximated by

$$
\begin{aligned}
& \partial_{t} \kappa=-\varsigma_{\lambda} \kappa-\mathcal{F}_{\lambda, \mathrm{b}}^{\mathrm{bo}}\left[\begin{array}{c}
\bar{z}_{\mathrm{i}} \\
\kappa
\end{array}\right] \\
& \partial_{t} \mathbf{M}_{\mathrm{ii}} \bar{z}_{\mathrm{i}}=-\nu \mathbf{S}_{\mathrm{ii}} \bar{z}_{\mathrm{i}}-\left[\begin{array}{ll}
\mathbf{L}_{\lambda, \mathrm{ii}} & \mathbf{L}_{\lambda, \mathrm{ib}}
\end{array}\right] \bar{z}-\left(\nu \mathbf{S}_{\mathrm{ib}}+\partial_{t} \mathbf{M}_{\mathrm{ib}}\right) B_{\bar{\Psi}}^{\Gamma} \kappa, \\
& \text { with } \overline{\mathcal{F}_{\lambda, \mathrm{b}}^{\text {bo }}}:=\left[\begin{array}{ll}
0_{M \times\left(s_{\mathbf{p}}-s_{\mathbf{e}}\right)} & \mathbf{I}_{M}
\end{array}\right] \overline{\mathcal{F}_{\lambda}^{\text {bo }}}\left[\begin{array}{cc}
\mathbf{I}_{s_{\mathbf{p}}-s_{\mathbf{e}}} & -\overline{B_{\Psi}} \\
0 & \mathbf{I}_{M}
\end{array}\right] \text {. }
\end{aligned}
$$


8.2. Discretization in time. Now to be able to solve numerically $(86)$ and $(87)$ in a given time interval $[0, T]$, with $T>0$, we need to discretize $[0, T]$. We introduce a uniform mesh $[0, T]_{D}$ consisting of $N_{t}+1 \geq 3$ points

$$
[0, T]_{D}:=\left(0, k T, 2 k T, \cdots,\left(N_{t}-1\right) k T, T\right),
$$

where $k:=\frac{T}{N_{t}}$ is the time step. Any function $z \in C([0, T] \times \Omega)$ will be approximated by the values $z_{i}^{j}:=z\left(j k, \mathbf{p}_{i}\right)$ taken in $[0, T]_{D} \times \Omega_{D}$, that is, we essentially approximate $z=z(t, x)$ by a matrix $[z]=\left[z_{(i, j)}\right] \in \mathcal{M}_{N_{p} \times\left(N_{t}+1\right)}, z_{(i, j)}:=z_{i}^{j}$. Notice that in the $j$ th column, denoted $\bar{z}^{j}$, we have an approximation of $z$ at time $t=j k, \bar{z}^{j}=\overline{z(j k, \cdot)} \approx z(j k, \cdot)$.

We will use the Crank-Nicolson scheme taking, for $t_{f}>t_{i},\left.\partial_{t} z\right|_{\frac{t_{i}+t_{f}}{2}} \approx \frac{z\left(t_{f}\right)-z\left(t_{i}\right)}{t_{f}-t_{i}}$ and $z\left(\frac{t_{i}+t_{f}}{2}\right) \approx \frac{z\left(t_{f}\right)+z\left(t_{i}\right)}{2}$. For system $(86)$, we obtain

$$
\frac{\mathbf{M}_{\mathrm{ii}}}{k}\left(\bar{z}_{\mathrm{i}}^{j+1}-\bar{z}_{\mathrm{i}}^{j}\right)=-\nu \frac{\mathbf{S}_{\mathrm{ii}}}{2}\left(\bar{z}_{\mathrm{i}}^{j+1}+\bar{z}_{\mathrm{i}}^{j}\right)-\frac{\mathbf{L}_{\lambda, \mathrm{ii}}^{j} \bar{z}_{\mathrm{i}}^{j}+\mathbf{L}_{\lambda, \mathrm{i}}^{j+1} \bar{z}_{\mathrm{i}}^{j+1}}{2}-\frac{\mathbf{M}_{\mathrm{ii}} \overline{\mathcal{F}_{\lambda}^{j, \mathrm{in}}} \bar{z}_{\mathrm{i}}^{j}+\mathbf{M}_{\mathrm{ii}} \overline{\mathcal{F}_{\lambda}^{j+1, \mathrm{in}}} \bar{z}_{\mathrm{i}}^{j+1}}{2},
$$

where, recalling that $\mathbf{L}_{\lambda, \mathrm{ii}}$ and $\mathcal{F}_{\lambda}^{\text {in }}$ (may) do depend on time, we denote $\mathbf{L}_{\lambda, \mathrm{ii}}^{j}:=\mathbf{L}_{\lambda, \mathrm{ii}}(j k)$ and $\overline{\mathcal{F}_{\lambda}^{j, \text { in }}}:=\overline{\mathcal{F}_{\lambda}^{\text {in }}(j k)}$. We wish to find $z_{\mathrm{i}}^{j+1}$ once $z_{\mathrm{i}}^{j}$ is known, Since $z_{\mathrm{i}}^{j+1}$ is unknown we make the following linear extrapolations

$$
\mathbf{L}_{\lambda, \mathrm{ii}}^{j+1} \bar{z}_{\mathrm{i}}^{j+1} \approx 2 \mathbf{L}_{\lambda, \mathrm{ii}}^{j} \bar{z}_{\mathrm{i}}^{j}-\mathbf{L}_{\lambda, \mathrm{ii}}^{j-1} \bar{z}_{\mathrm{i}}^{j-1} \text { and } \overline{\mathcal{F}_{\lambda}^{j+1, \mathrm{in}}} \bar{z}_{\mathrm{i}}^{j+1} \approx 2 \overline{\mathcal{F}}_{\lambda}^{j, \mathrm{in}} \bar{z}_{\mathrm{i}}^{j}-\overline{\mathcal{F}}_{\lambda}^{j-1, \mathrm{in}} \bar{z}_{\mathrm{i}}^{j-1} .
$$

Defining $\mathbf{A}_{\mathrm{ii}}^{\oplus}:=\left(2 \mathbf{M}_{\mathrm{ii}}+k \nu \mathbf{S}_{\mathrm{ii}}\right)$ and $\mathbf{A}_{\mathrm{ii}}^{\ominus}:=\left(2 \mathbf{M}_{\mathrm{ii}}-k \nu \mathbf{S}_{\mathrm{ii}}\right)$, we obtain the system

$$
\mathbf{A}_{\mathrm{ii}}^{\oplus} \bar{z}_{\mathrm{i}}^{j+1}=\mathbf{A}_{\mathrm{ii}}^{\ominus} \bar{z}_{\mathrm{i}}^{j}-k\left(3 \mathbf{L}_{\lambda, \mathrm{ii}}^{j} \bar{z}_{\mathrm{i}}^{j}-\mathbf{L}_{\lambda, \mathrm{ii}}^{j-1} \bar{z}_{\mathrm{i}}^{j-1}\right)-k \mathbf{M}_{\mathrm{ii}}\left(3 \overline{\mathcal{F}_{\lambda}^{j, \mathrm{in}}} \bar{z}_{\mathrm{i}}^{j}-\overline{\mathcal{F}}_{\lambda}^{j-1, \mathrm{in}} \bar{z}_{\mathrm{i}}^{j-1}\right),
$$

which we invert to obtain $\bar{z}_{\mathrm{i}}^{j+1}$. To start the loop we define, at the "ghost" time instant $t=-k T$, the terms $\mathbf{L}_{\lambda, \mathrm{ii}}^{-1} \bar{z}_{\mathrm{i}}^{-1}:=\mathbf{L}_{\lambda, \mathrm{ii}}^{0} \bar{z}_{\mathrm{i}}^{0}$ and $\overline{\mathcal{F}_{\lambda}^{-1, \mathrm{in}}} \bar{z}_{\mathrm{i}}^{-1}:=\overline{\mathcal{F}_{\lambda}^{0, \text { in }}} \bar{z}_{\mathrm{i}}^{0}$.

Remark 8.6. Notice that since $\mathbf{M}_{\mathrm{ii}}$ and $\mathbf{S}_{\mathrm{ii}}$ are symmetric and positive definite, then $\mathbf{A}_{\mathrm{ii}}^{\oplus}$ is symmetric and positive definite (at least for small enough $k$ ). Notice also that the idea in (89) is proposed in [KR15b, section 5.4], but with an extrapolation as $L^{j+1} \approx$ $(1+k) L^{j}-k L^{j-1}$, which approaches a zero order extrapolation as $k$ decreases to 0 . With the linear extrapolation, we observed a better accuracy/convergence performance in some tests. Finally, the feedback part of the control in [KR15b, section 5.4] is treated in a different way using a preliminary "uncontrolled guess" $z_{G}^{j+1}$ for $\bar{z}_{\mathrm{i}}^{j+1}$ by solving the system with no control, the idea is to use the fact that we know $\mathcal{F}^{j+1 \text {,in }}$. We do not use this fact with the linear extrapolation above. However, in the 2D boundary case, the extrapolation seems to work better in our simulations, this is why we take the extrapolation in both cases. Furthermore, the extrapolation approach is cheaper because we need to solve the system only once (at each time step).

Analogously, system $87 \mathrm{a}$ is approximated by

$$
\left(2+k \varsigma_{\lambda}\right) \kappa^{j+1}=\left(2-k \varsigma_{\lambda}\right) \kappa^{j}-k\left(3 \overline{\mathcal{F}_{\lambda, \mathrm{b}}^{j, \mathrm{bo}}}\left[\begin{array}{l}
\bar{z}_{\mathrm{i}}^{j} \\
\kappa^{j}
\end{array}\right]-\overline{\mathcal{F}_{\lambda, \mathrm{b}}^{j-1, \mathrm{bo}}}\left[\begin{array}{c}
\bar{z}_{\mathrm{i}}^{j-1} \\
\kappa^{j-1}
\end{array}\right]\right)
$$

with $\overline{\mathcal{F}_{\lambda, \mathrm{b}}^{-1, \mathrm{bo}}}\left[\begin{array}{c}\bar{z}_{\mathrm{i}}^{-1} \\ \kappa^{-1}\end{array}\right]:=\overline{\mathcal{F}_{\lambda, \mathrm{b}}^{0, \mathrm{bo}}}\left[\begin{array}{l}\bar{z}_{\mathrm{i}}^{0} \\ \kappa^{0}\end{array}\right]$, from which we obtain $\kappa^{j+1}$. Then, system $87 \mathrm{~b}$ is approximated by

$$
\begin{aligned}
\mathbf{A}_{\mathrm{ii}}^{\oplus} \bar{z}_{\mathrm{i}}^{j+1}= & \mathbf{A}_{\mathrm{ii}}^{\ominus} \bar{z}_{\mathrm{i}}^{j}-k \nu \mathbf{S}_{\mathrm{ib}} B \bar{\Psi}_{\bar{\Psi}}^{\Gamma}\left(\kappa^{j+1}+\kappa^{j}\right)-2 \mathbf{M}_{\mathrm{ib}} B_{\bar{\Psi}}^{\Gamma}\left(\kappa^{j+1}-\kappa^{j}\right) \\
& -k \mathbf{L}_{\lambda, \mathrm{ii}}^{j+1} \bar{z}_{\mathrm{i}}^{j+1}-k \mathbf{L}_{\lambda, \mathrm{ii}}^{j} \bar{z}_{\mathrm{i}}^{j}-k \mathbf{L}_{\lambda, \mathrm{ib}}^{j+1} B_{\bar{\Psi}}^{\Gamma} \kappa^{j+1}-k \mathbf{L}_{\lambda, \mathrm{ib}}^{j} B_{\bar{\Psi}}^{\Gamma} \kappa^{j} .
\end{aligned}
$$


Extrapolating again for the unknown $\mathbf{L}_{\lambda, \mathrm{ii}}^{j+1} \bar{z}_{\mathrm{i}}^{j+1}$, with $\mathbf{L}_{\lambda, \mathrm{ii}}^{-1} \bar{z}_{\mathrm{i}}^{-1}:=\mathbf{L}_{\lambda, \mathrm{ii}}^{0} \bar{z}_{\mathrm{i}}^{0}$, we arrive to

$$
\begin{aligned}
\mathbf{A}_{\mathrm{ii}}^{\oplus} \bar{z}_{\mathrm{i}}^{j+1}= & \mathbf{A}_{\mathrm{ii}}^{\ominus} \bar{z}_{\mathrm{i}}^{j}-\left(\mathbf{A}_{\mathrm{ib}}^{\oplus}+k \mathbf{L}_{\lambda, \mathrm{ib}}^{j+1}\right) B \bar{\Psi}_{\bar{T}}^{\Gamma} \kappa^{j+1}+\left(\mathbf{A}_{\mathrm{ib}}^{\ominus}-k \mathbf{L}_{\lambda, \mathrm{ib}}^{j}\right) B \Gamma_{\bar{\Psi}}^{\Gamma} \kappa^{j} \\
& -k 3 \mathbf{L}_{\lambda, \mathrm{ii}}^{j} \bar{z}_{\mathrm{i}}^{j}+k \mathbf{L}_{\lambda, \mathrm{ii}}^{j-1} \bar{z}_{\mathrm{i}}^{j-1},
\end{aligned}
$$

which we invert to obtain the interior component $\bar{z}_{\mathrm{i}}^{j+1}$ of $\bar{z}^{j}$. Notice that the boundary component is given by $\bar{z}_{\mathrm{b}}^{j+1}=B \frac{\Gamma}{\Psi} \kappa^{j+1}$.

8.2.1. Solving the Riccati systems. It remains to explain how we compute the the feedbacks $\overline{\mathcal{F}_{\lambda}^{\text {in }}}$ and $\overline{\mathcal{F}_{\lambda}^{\text {bo }}}$ we need in (90) and (91a). That is, to explain how we solve the Ricatti system (84), backwards in time and in an bounded interval of time $[0, T]$. We follow the procedure in [KR15b, sections 5.3.2 and 5.3.3], with some changes.

Internal feedback. Firstly, we look for a solution $\Pi_{D}^{T}$ of the agebraic Riccati equation

$$
\Pi_{D}^{T} \mathbf{X}_{\lambda}^{\text {in }}(T)+\mathbf{X}_{\lambda}^{\text {in }}(T)^{\top} \Pi_{D}^{T}-\Pi_{D}^{T} \mathbf{R} \mathbf{R}^{\top} \Pi_{D}^{T}+\left(\mathbf{C}^{\text {in }}\right)^{\top}\left(\mathbf{C}^{\text {in }}\right)=0
$$

with $\mathbf{R}=\mathbf{H}_{0}=\mathbf{M}_{\mathrm{ii}, \mathrm{c}}^{-1}$ as the Cholesky factor of $\mathbf{M}_{\mathrm{ii}}^{-1}$. The main idea is to have $\mathbf{H}_{0} \mathbf{H}_{0}^{\top}=$ $\mathbf{M}_{\mathrm{ii}}$ which corresponds to taking the identity as control operator (see [KR15b, 5.3.3] where it is chosen $\left.\mathbf{H}_{0}=\left(\mathbf{M}_{\mathrm{ii}, \mathrm{c}} \mathbf{M}_{\mathrm{ii}}^{-1}\right)^{\top}\right)$.

Secondly, we connect $\mathbf{H}_{0}$ to $\mathbf{R}^{\text {in }}$ by an homotopy. In the case where we have finitedimensional controls $\mathbf{R}^{\text {in }}$ is a rectangular matrix, so we add the enough zero columns to obtain a square matrix, and we consider

$$
\mathbf{H}_{\tau}=(1-\tau)^{2} \mathbf{H}_{0}+\tau^{2}\left[\mathbf{R}^{\mathrm{in}} 0\right], \quad \tau \in[0,1] .
$$

Discretizing the homotopy interval $[0,1]_{D}=\left[0, l, 2 l, \ldots,\left(N_{h}-1\right) l, 1\right], N_{h} \in \mathbb{N}_{0}$ and $l=\frac{1}{N_{h}}$, we compute the solution corresponding to $\mathbf{R}=\mathbf{H}_{m l}$ from those corresponding to $\mathbf{R}=$ $\mathbf{H}_{(m-1) l}, m \in\left\{1,2, \ldots, N_{h}\right\}$, following $\left[\mathrm{KR} 15 \mathrm{~b}\right.$. Let $\Pi_{D}^{T}$ be the solution corresponding to $\mathbf{H}_{1}$, then since $\mathbf{H}_{1} \mathbf{H}_{1}^{\top}=\mathbf{R}^{\mathrm{in}} \mathbf{R}^{\mathrm{in} T}, \Pi_{D}^{T}$ is the solution corresponding to $\mathbf{R}=\mathbf{R}^{\mathrm{in}}$.

Finally, we solve the equation (84) as in [KR15b], with the final condition $\Pi_{D}(T)=\Pi_{D}^{T}$ and using Crank-Nicolson discretization in time variable, by transforming the equation into an algebraic Riccati equation at each time step.

To solve the algebraic Riccati equations we use the software in [Ben] (see also [Ben06]).

Remark 8.7. Notice that in KR15b the solution $\Pi_{D}^{T}$ is found in three steps and $\mathbf{R}$ is always set to be a square matrix, this is because we need to connect two matrices by an homotopy. Here we find $\Pi_{D}^{T}$ in two steps and we implicitly connect the square matrix $\mathbf{H}_{0}$ to the rectangular one $\mathbf{R}^{\text {in }}$ by connecting $\mathbf{H}_{0}$ to $\left[\begin{array}{ll}\mathbf{R}^{\text {in }} & 0\end{array}\right]$. We have used the homotopy (92) because in some situations we observed that we need less homotopy steps than with the convex combination $(1-\tau) \mathbf{H}_{0}+\tau\left[\mathbf{R}^{\text {in }} \quad 0\right]$ like as in [KR15b], but we cannot say a priori which homotopy is better.

Boundary feedback. We proceed as in the internal case to find a final condition for the differential Riccati equation. At final time $t=T$ we start by solving the system

$$
\Pi_{D}^{T} \mathbf{X}_{\lambda}^{\mathrm{bo}}(T)+\mathbf{X}_{\lambda}^{\mathrm{bo}}(T)^{\top} \Pi_{D}^{T}-\Pi_{D}^{T} \mathbf{R} \mathbf{R}^{\top} \Pi_{D}^{T}+\left(\mathbf{C}^{\mathrm{bo}}\right)^{\top}\left(\mathbf{C}^{\mathrm{bo}}\right)=0
$$

and then set $\mathbf{H}_{0}=\left[\begin{array}{cc}\mathbf{M}_{\mathrm{ii,c}}^{-1} & 0 \\ 0 & \mathbf{I}_{M}\end{array}\right]$ and $\mathbf{H}_{\tau}=(1-\tau)^{2} \mathbf{H}_{0}+\tau^{2}\left[\begin{array}{ll}\mathbf{R}^{\mathrm{bo}} & 0\end{array}\right], \tau \in[0,1]$. 


\section{Numerical EXAMPles}

We present some results of numerical simulations which we have performed concerning the stabilization of systems (80) with internal feedback control or (81) with boundary feedback control to zero. Below, $z_{\mathrm{u}, 0}$ stands for the solution of the uncontrolled discretized systems (i.e., without the feedback term and $\lambda=0$ ), and $z_{\lambda}$ stands for the solution of the discretized systems under the action of a discretized feedback control. We focus on the $2 \mathrm{D}$ case and our domain is the unit ball. In the internal feedback control case, we define a rectangle subdomain $\omega:=\left(0, \frac{1}{2}\right) \times\left(0, \frac{1}{3}\right)$. Then, we take a regular partition of into $M=m n$ subrectangles

$$
\omega_{l_{1}, l_{2}}:=\left(\frac{l_{1}-1}{2 m}, \frac{l_{1}}{2 m}\right) \times\left(\frac{l_{2}-1}{3 n}, \frac{l_{2}}{3 n}\right), \quad\left(l_{1}, l_{2}\right) \in\{1,2, \ldots, m\} \times\{1,2, \ldots, n\} .
$$

We take the $M$ actuators $1_{\omega_{l_{1}, l_{2}}}$, thus in each subrectangle $\omega_{l_{1}, l_{2}}$ the control is constant. As an illustration, we plot a linear combination of 4 piecewise-constant actuators in Figure 1(a), corresponding to the arrangement $(m, n)=(2,2)$. For the boundary control case, our boundary, once parametrized by arc length, is $\Gamma=[0,2 \pi)$. We use boundary actuators whose form is

$$
\Psi_{i}(\theta)=1_{\left(\theta_{0}, \theta_{1}\right)} \sin \left(\frac{i\left(\theta-\theta_{0}\right)}{\theta_{1}-\theta_{0}}\right) .
$$

with $\theta_{0}=\pi$ and $\theta_{1}=\frac{5 \pi}{4}$. As an illustration, the boundary actuator $\Psi_{2}$ is plotted in Figure 1(b).

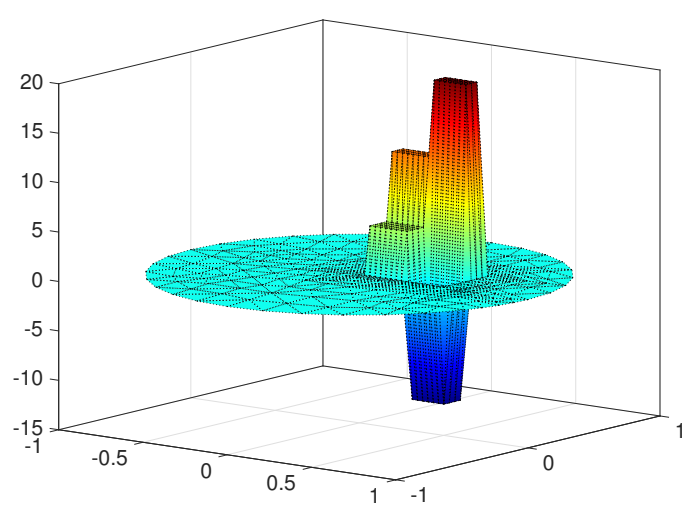

(a) A linear combination off 4 piecewise-constant internal actuators.

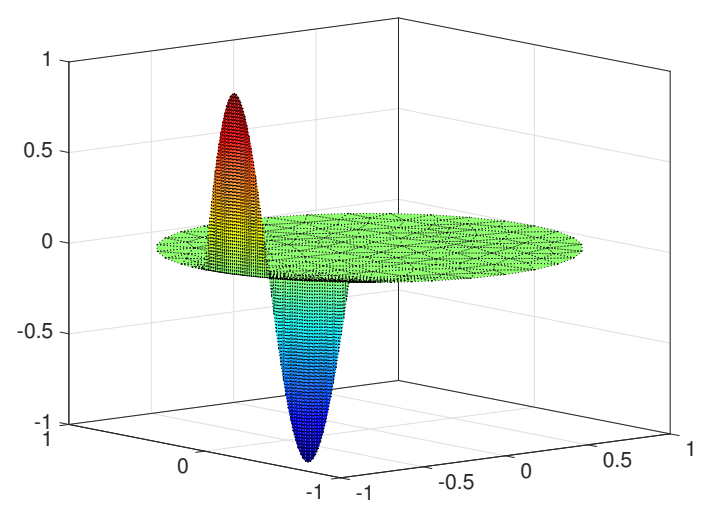

(b) The boundary actuator $\Psi_{2}$.

FiguRE 1. Internal and boundary actuators.

9.1. Testing with a family of functions $(\hat{a}, \hat{b})$. We set $\lambda=2, \nu=\frac{1}{4},, \chi=\mathbb{1}_{\omega}$. Next, we choose a family of functions $\hat{a}, \hat{b}_{1}$, and $\hat{b}_{2}$ as follows

$$
\begin{aligned}
\hat{a}(t, x) & =-\sin (t) \cos \left(\mathbf{i} x_{1}\right)+\sin (5 t) \sin \left(\mathbf{j} x_{2}\right)-3, \\
\hat{b}_{1}(t, x) & =\cos (t) \sin \left(-\mathbf{k} x_{1}\right)-\cos (3 t) \cos \left(\mathbf{l} x_{2}\right), \\
\hat{b}_{2}(t, x) & =\sin (-t) \sin \left(\mathbf{m} x_{1}\right)-\cos (2 t) \sin \left(\mathbf{n} x_{2}\right) .
\end{aligned}
$$

We will test firstly with 6 piecewise-constant actuators, corresponding to $(m, n)=(3,2)$, and then with 6 boundary actuators $\Psi_{i}, i \in\{1,2, \ldots, 6\}$. The initial condition is set $v_{0}(x):=\sin \left(2 x_{1}\right) \cos \left(x_{2}\right)$ and the time-interval is set $[0,8]$. In Figures $2(\mathrm{~b})$ and $2(\mathrm{c})$, we 


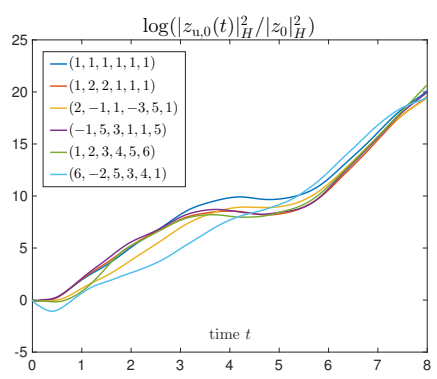

(a) Without control.

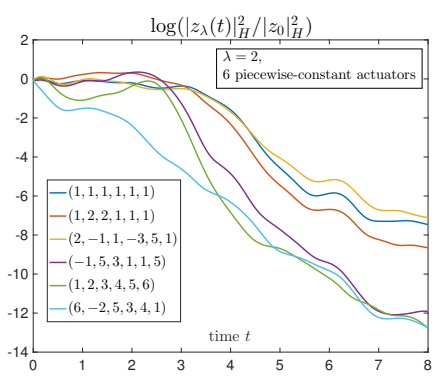

(b) With internal feedback.

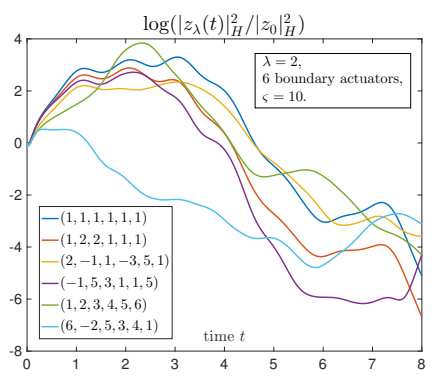

(c) With boundary feedback.

FiguRE 2. Stabilization rate is provided by the feedback control.

can observe that both internal and boundary feedback control is able to stabilize the system with the desired rate $\frac{\lambda}{2}=1$ for some parameters $(\mathbf{i}, \mathbf{j}, \mathbf{k}, \mathbf{l}, \mathbf{m}, \mathbf{n}) \in \mathcal{P}$, with

$$
\begin{aligned}
\mathcal{P}:= & \{(1,1,1,1,1,1),(1,2,2,1,1,1),(2,-1,1,-3,5,1), \\
& (-1,5,3,1,1,5),(1,2,3,4,5,6),(6,-2,5,3,4,1)\} .
\end{aligned}
$$

In Figure 2, as well in following ones, the squared norm $|z|_{H}^{2}$ is understood as the discrete approximation $\bar{z}^{\top} \mathbf{M} \bar{z}$. We would like to emphasize that without any control, the system is unstable for these parameters defined by $\mathcal{P}$ as we can see from Figure 2 (a).

9.2. Increasing the number of actuators. We compare the results we obtain by changing the number of actuators. With no surprise, we see that with more actuators we obtain better results. We will take one element of the family in (94), namely the one corresponding to $(\mathbf{i}, \mathbf{j}, \mathbf{k}, \mathbf{l}, \mathbf{m}, \mathbf{n})=(2,-1,1,-3,5,1)$ and also take $\lambda=2$.

9.2.1. Internal feedback control. In Figure 3, we present the results for some rearrangements $(m, n)$. We observe that the cost $(\Pi z(t), z(t))_{H}$, understood as $\overline{z(t)}{ }^{\perp} \Pi_{D}(t) \overline{z(t)}$, decreases as the number of controls increase, as we can see in Figure $3(\mathrm{~b})$.

Notice that we cannot say a priori which among $(m, n)=(2,2)$ and $(m, n)=(4,1)$ is better because one set of actuators does not include the other. However, for the considered example we observe that $(2,2)$ is better than $(4,1)$.

The case $(m, n)=(+\infty,+\infty)$ in Figure 3 should be understood as the case we do not impose any restriction on number of actuators. That is, to the case we take $P_{M}=1$, or in other words in the case our control operator in (33) is just $1_{\omega} \chi 1_{\omega}$. In this case $\mathbf{R}^{\text {in }}=\mathcal{D}_{\overline{\chi 1_{\omega}}}$ in 85 .

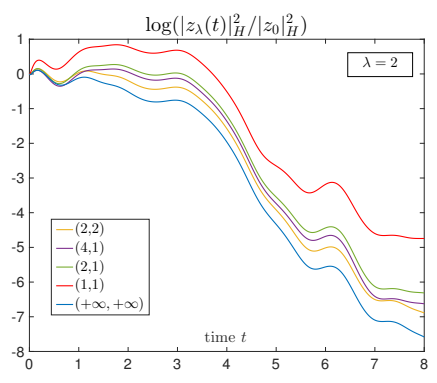

(a) Controlled solution.

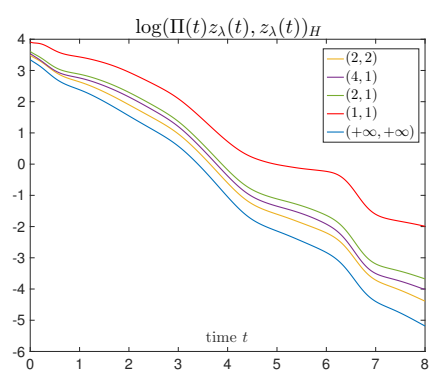

(b) Cost function.

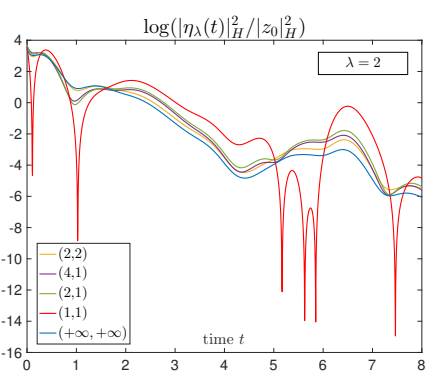

(c) Control.

FiguRE 3. The convergence rate of the solution, the cost function and the control. 
Recall that our control can be written as $\eta(t, x)=\sum_{l_{1}=1}^{m} \sum_{l_{2}=1}^{n} \eta_{l_{1}, l_{2}}(t) 1_{\omega_{l_{1}, l_{2}}}(x)$. For example, in the left column of Figure 4, we plot the value of the feedback control, in the case of a single actuator, at two time instants $t=4.5$ and $t=6.5$. We refer also to Figure 5 (a) for this value at all time instants $t \in[0,8]$. Notice that, the "cusps" in Figure 3 (c), in the case $(m, n)=(1,1)$, are due to the fact that the control vanishes at the corresponding time instants. In the case we take only one actuator a "cusp" will appear when the control changes sign, because the plotted function "takes" the value $-\infty$ if the control vanishes, see also Figure 5(a).

In Figure 4, we plot the control at two time instants $t=4.5$ and $t=6.5$. In Figure 5 we plot the value of each actuator for all time instants for some rearrangements $(m, n)$.
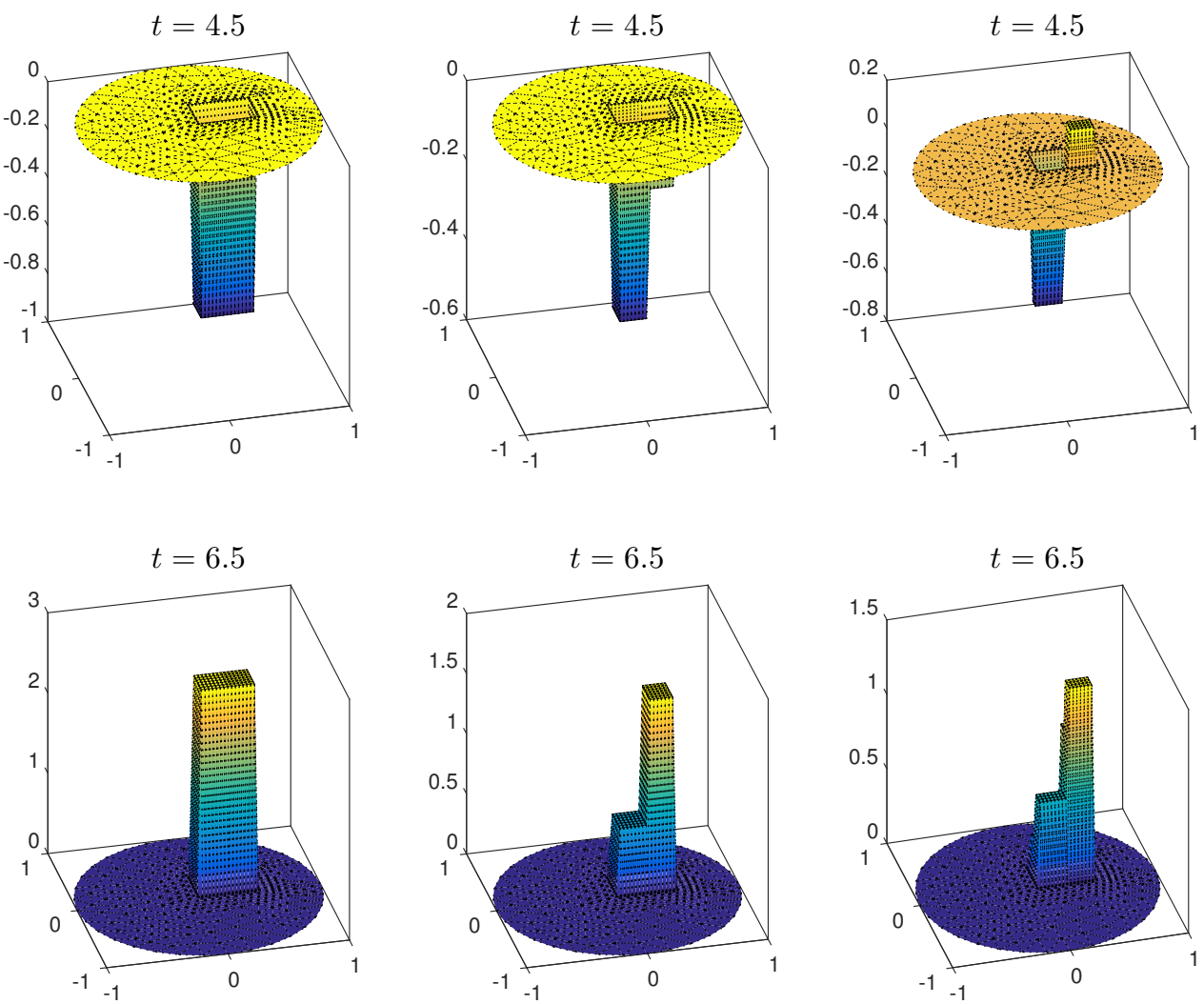

Figure 4. Feedback control at $t \in\{4.5,6.5\}$ for $(m, n) \in\{(1,1),(2,1),(2,2)\}$.

9.2.2. Boundary feedback control. Now we consider the reference function in 9.1 corresponding to $(\mathbf{i}, \mathbf{j}, \mathbf{k}, \mathbf{l}, \mathbf{m}, \mathbf{n})=(6,-2,5,3,4,1)$ and we take $\lambda=2$ and $\varsigma=10$. We take a family of actuators $\left\{\Psi_{i} \mid i \in\{1,2, \ldots, M\}\right\}$, as in (93). We compare the result in the cases $M \in\{1,2,4,6\}$. With no surprise, from Figure 6(a), taking more actuators leads to better results. The boundary feedback control here can be written as $\eta_{M}(t)=\sum_{i=1}^{M} \kappa_{i}(t) \Psi_{i}$. In Figures 6 (b,c) we plot the functions $\kappa_{i}=\kappa_{i}(t, M)$ for the cases $M \in\{1,6\}$.

9.3. Comparing with another placement of the actuators. We consider another placement of the actuators and compare the controlled solutions. We recall one member 


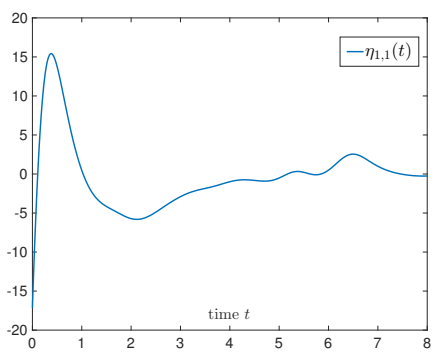

(a) $(m, n)=(1,1), 1$ actuator.

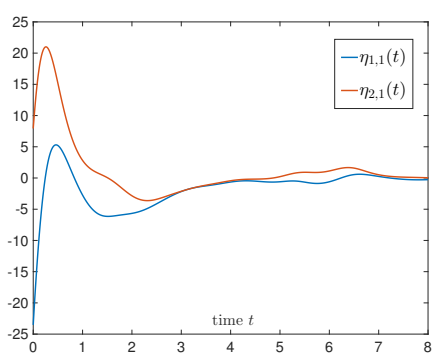

(b) $(m, n)=(2,1), 2$ actuators.

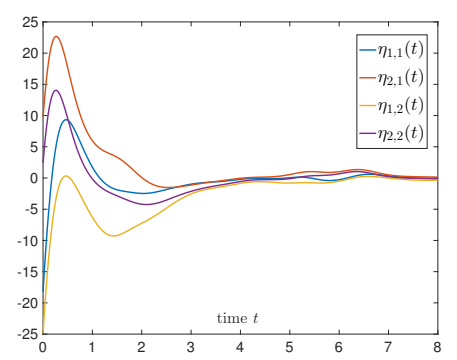

(c) $(m, n)=(2,2), 4$ actuators.

Figure 5. The magnitude(s) of the internal actuator(s).

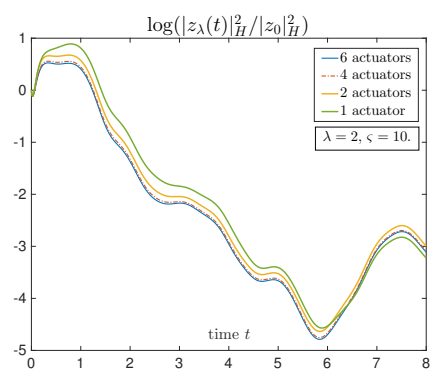

(a) The cases $M \in\{1,2,4,6\}$.

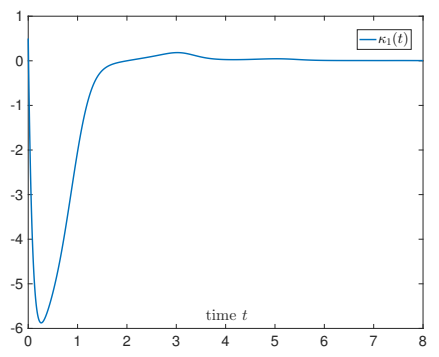

(b) Magnitude for 1 actuator.

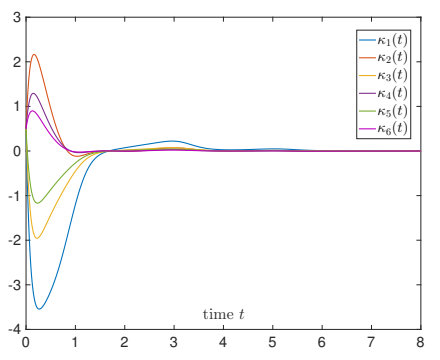

(c) Magnitudes for 6 actuators.

Figure 6. Convergence rate of solutions and the magnitude(s) of the boundary actuator(s).

of the family in (94), namely the one corresponding to $(\mathbf{i}, \mathbf{j}, \mathbf{k}, \mathbf{l}, \mathbf{m}, \mathbf{n})=(1,2,2,1,1,1)$ and also set $\lambda=2$.

9.3.1. Internal feedback control. Instead of defining all actuators in one rectangle $\omega=$ $\left(0, \frac{1}{2}\right) \times\left(0, \frac{1}{3}\right)$ as in Figure 1(a), we define 4 piecewise-constant actuators in 4 rectangles $\omega_{i}$ away from each other as in Figure 7(a). We want to say that the sum of the areas of the four separated rectangles $\omega_{i}$ equals the area of $\omega$, that is, $\sum_{i=1}^{4}\left|\omega_{i}\right|=|\omega|$. Comparing Figures 7 (b) and 3(a), we see that the latter placement is more effective than the former one (for this example).

9.3.2. Boundary feedback control. Here, we set $\zeta=10$ and we use 4 boundary actuators. Instead of defining as in (93), see Figure 1(b), using 4 frequencies of the sinus functions in an interval $\left(\pi, \frac{5 \pi}{4}\right)$, we now define the new actuators in domains away from each other as in Figure 8(a), where each actuator is as in (93) with $i=1$. Comparing Figures 8(b) and 6(a) we see that the latter placement seems to be better than the former one (for this example).

9.4. Dependence of the transient bound on the desired decreasing rate. Here we consider the case of internal controls with no restriction in the dimension of the control, we take $P_{M}=1, \chi=1$ and $\mathbf{R}^{\text {in }}=\mathcal{D} \overline{\chi_{1 \omega}}$ in $(85)$. We want to check whether the transient bound $\widehat{C}_{\lambda}$ associated to the Riccati based feedback control does depend on the desired exponential rate $\lambda \geq 0$ as

$$
C_{\lambda} \approx \mathrm{e}^{\mathcal{T}_{0, \alpha}+\mathcal{T}_{1, \alpha} \lambda^{\alpha}}
$$

(cf. Section 3.6), for a suitable $\alpha \geq 0$. As we will see, the answer is not clear. 


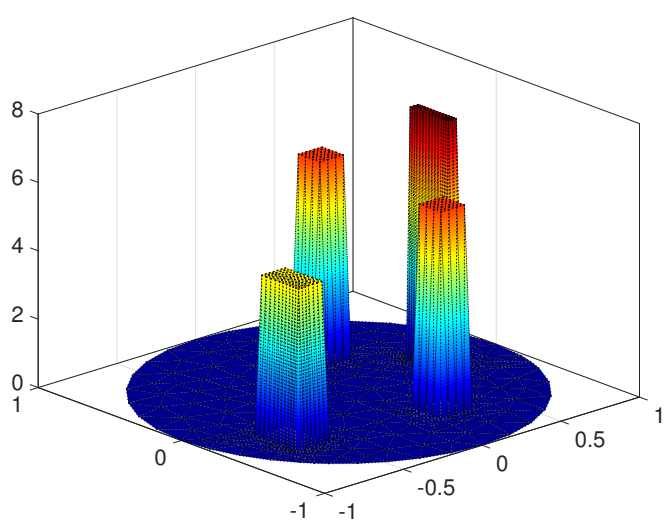

(a) New placement of 4 internal actuators.

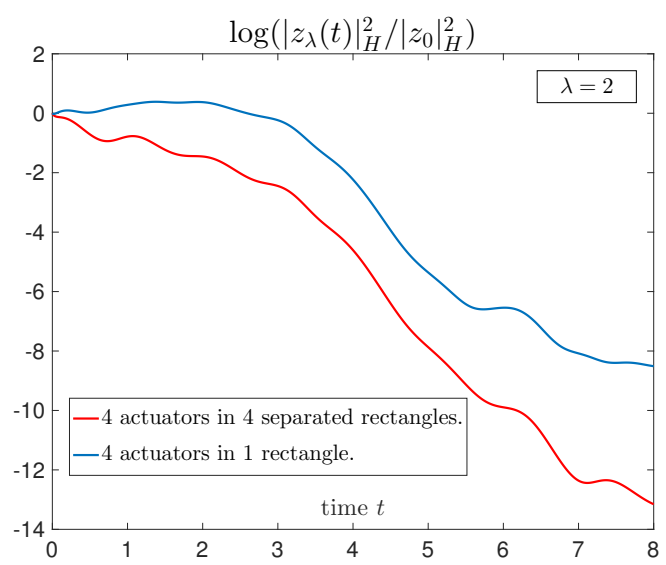

(b) Controlled solution of the 2 placements.

FiguRE 7. Compare two placements of internal actuators.

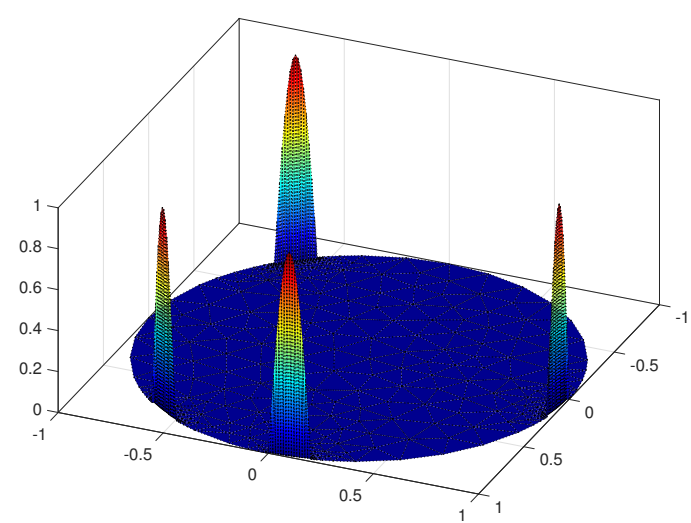

(a) New placement of 4 boundary actuators.

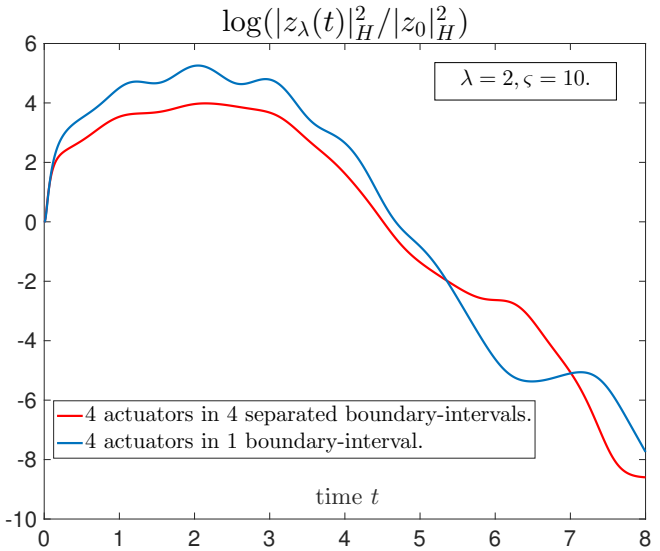

(b) Controlled solution of the two placements.

FiguRE 8. Compare two placements of the boundary actuators.

We set $\nu=\frac{1}{2}, \hat{a}=-10+2 x_{1}+\cos x_{2}, \hat{b}=\left(-x_{1}^{2},-\sin x_{2}\right)$, and we impose no restriction on number of actuators.

Notice that $(\hat{a}, \hat{b})$ is independent of time. In the stationary case, we have "just" to solve an algebraic Riccati equation for each $\lambda$. Up to our best knowledge, even in the stationary case it is not known how precisely the constant $C_{\lambda}$ depends on $\lambda$, for Riccati based stabilizing feedback.

Figure 9 shows that the system can be stabilized with rate $\lambda \in[0,30]$, the figure also shows that the constant $C_{\lambda}$ increases with $\lambda$.

Let us observe that starting at time $t=s_{0}<T$, if for all $t \in\left[s_{0}, T\right]$ we have $|z(t)|_{H}^{2} \leq$ $C_{\lambda, s_{0}} \mathrm{e}^{-\lambda\left(t-s_{0}\right)}\left|z\left(s_{0}\right)\right|_{H}^{2}$, then we obtain the lower bound estimate

$$
C_{\lambda} \geq C_{\lambda, s_{0}} \geq \max _{t \in\left[s_{0}, T\right]} \mathrm{e}^{\lambda\left(t-s_{0}\right)} \frac{|z(t)|_{H}^{2}}{\left|z\left(s_{0}\right)\right|_{H}^{2}}
$$




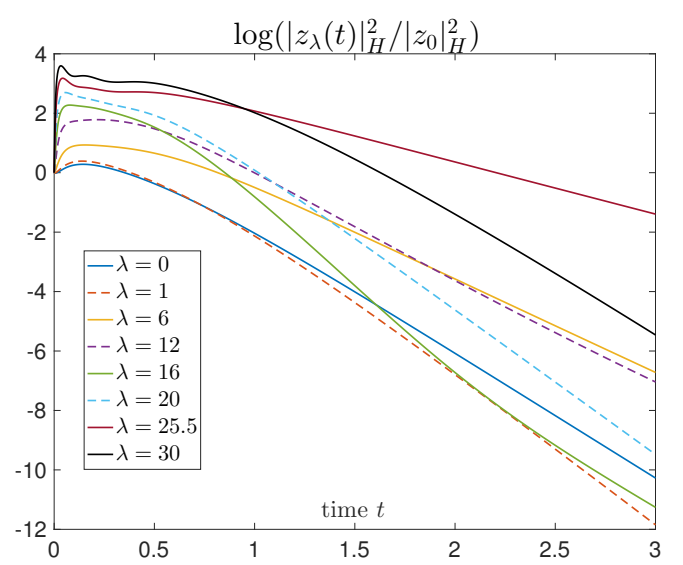

(a) With internal feedback control.

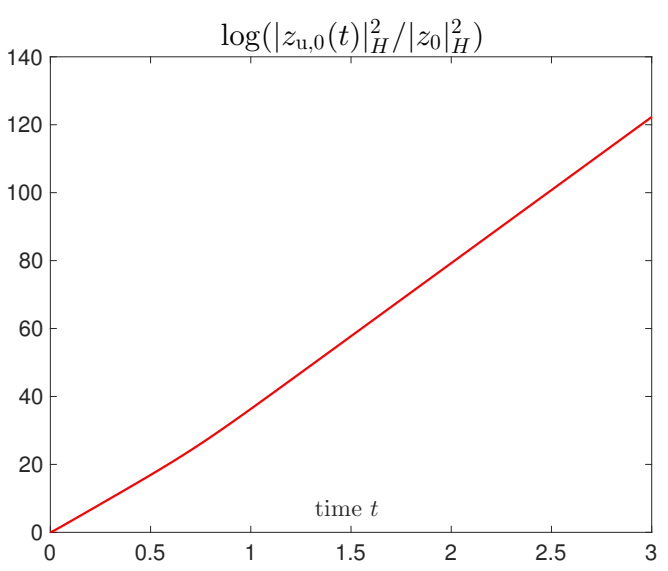

(b) Without control.

FiguRE 9. Stabilization rate of the solution is achieved under feedback control.

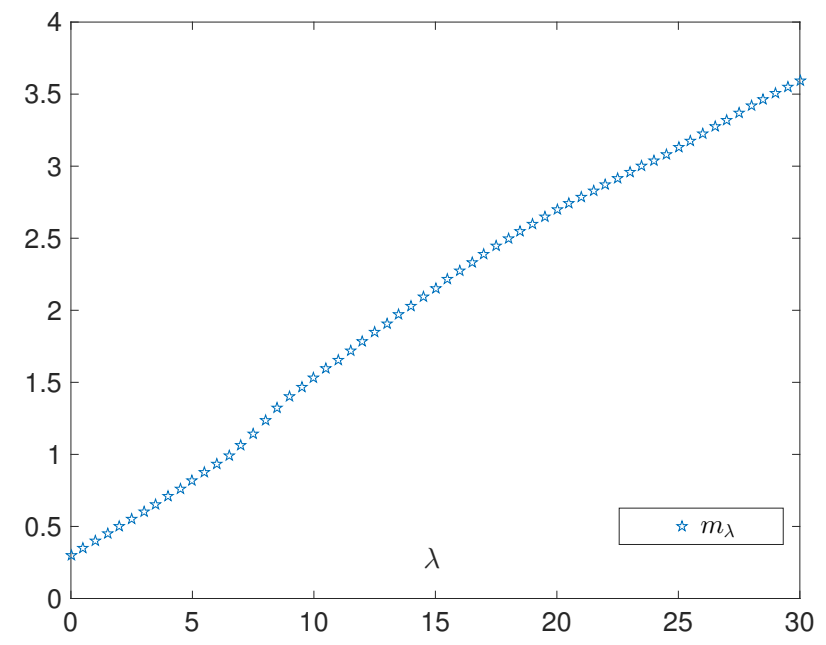

FiguRE 10. $m_{\lambda}$ with $\lambda \in\left\{0, \frac{1}{2}, 1, \frac{3}{2}, \ldots, 29,29 \frac{1}{2}, 30\right\}$.

and, considering all $s_{0} \in[0, T)$ we obtain

$$
\begin{aligned}
\log C_{\lambda} \geq m_{\lambda} & :=\max _{s_{0} \geq 0} \max _{t \in\left[s_{0}, T\right]}\left(\lambda\left(t-s_{0}\right)+\log \left(\frac{|z(t)|_{H}^{2}}{\left|z\left(s_{0}\right)\right|_{H}^{2}}\right)\right) \\
& =\max _{s_{0} \geq 0} \max _{t \in\left[s_{0}, T\right]}\left(r_{\lambda}(t)-r_{\lambda}\left(s_{0}\right)\right),
\end{aligned}
$$

with $r_{\lambda}(t):=\lambda t+\log \left(\frac{|z(t)|_{H}^{2}}{\left|z_{0}\right|_{H}^{2}}\right)$. In Figure 10 we plot the function $m_{\lambda}$, which suggests that (95) might hold with $\alpha \in(0,1]$, but we cannot confirm that (41) will hold for "big $\lambda$ ", actually we cannot even say whether $\lambda=30$ is big. Notice that for $\lambda \in[20,30]$ the figure suggests that $\alpha=1$ is the best fitting parameter. We must however say that we expect the magnitudes of control and solution to increase as $\lambda$ does, so we must be careful in reading the results for big $\lambda$, because the mesh used was the same for all $\lambda$, and for bigger $\lambda$ the numerical error will be more significant.

We present another example in the nonautonomous case. Notice that, this case is quite expensive, because we need to solve a differential Riccati equation for each $\lambda$. Here, we set $\nu=\frac{1}{4}, \hat{a}=-10+2 x_{1}+\sin 2 t \cos x_{2}, \hat{b}=\left(-x_{1}^{3} \cos 3 t,-\sin t \sin x_{2}\right)$, and we impose 


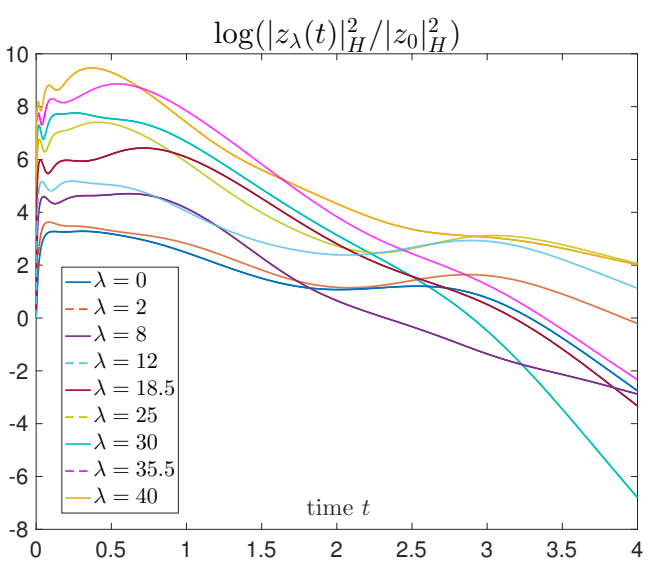

(a) With internal feedback control.

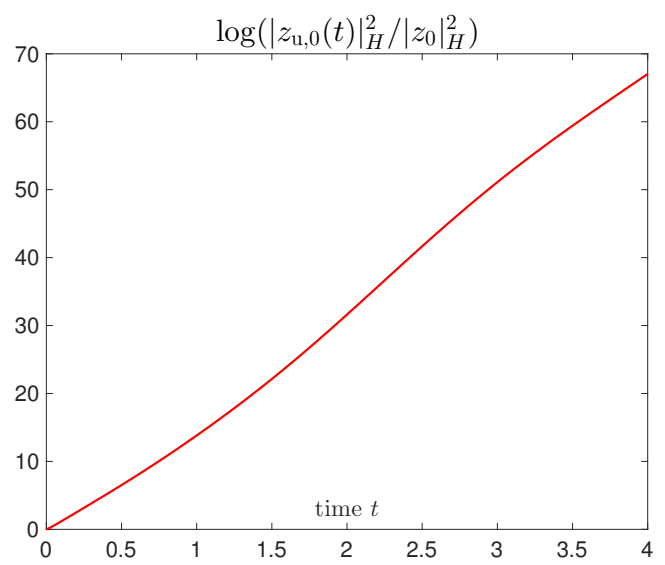

(b) Without control.

FiguRE 11. Stabilization rate of the solution is achieved under feedback control.

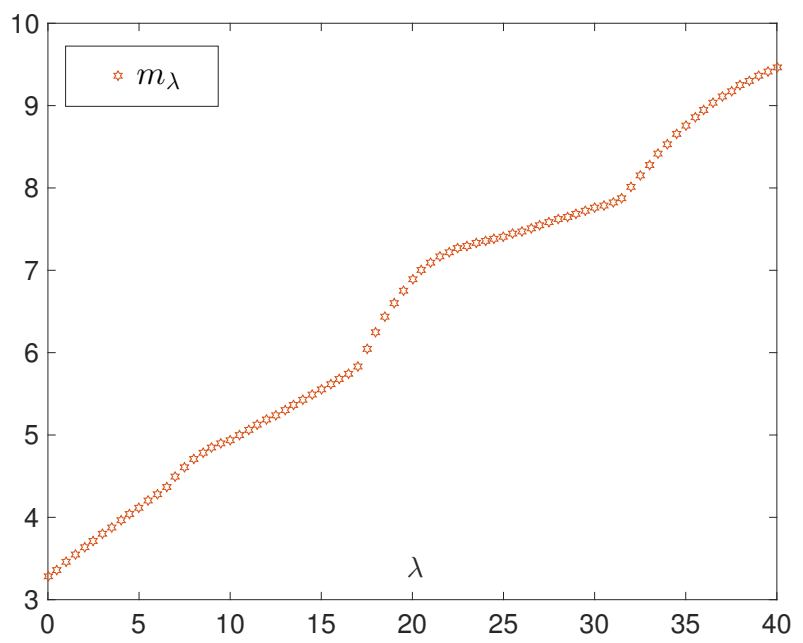

FiguRE 12. $m_{\lambda}$ with $\lambda \in\left\{0, \frac{1}{2}, 1, \frac{3}{2}, \ldots, 39,39 \frac{1}{2}, 40\right\}$.

no restriction on number of actuators. Figures 11 and 12 show that the system can be stabilized with rate $\lambda \in[0,40]$ and the constant $C_{\lambda}$ increases with $\lambda$. Again the Riccati feedback we use seems not to provide the behaviour (41) "for big $\lambda$ ". So one question still remain: how small can we make the transient bound $C_{\lambda}$ and/or the ratio $\frac{C_{\lambda}}{\lambda}$ (cf. Section 3.6). Which feedback (Riccati based or not) makes the ratio smaller?

In conclusion, the dependence of the transient bound $\widehat{C}_{\lambda}$, associated to the Riccati based feedback control, on the desired exponential rate $\lambda \geq 0$ is not clear yet. This could be the subject of further researh and more simulations must be done, in particular for finer meshes. This is however not trivial, because solving the Riccati equations is numerically quite demanding for fine meshes. However, the simulations strongly suggest that (unfortunately) $\widehat{C}_{\lambda}$ depends exponentially-like on $\lambda$, which suggests us that to treat the nonlinear system we should take $\lambda$ relatively small, because $\epsilon$ in (3) will decrease as $\widehat{C}_{\lambda}$ increases (cf.Remark 4.10).

9.5. On the parameter $\varsigma$. Here we consider the case of boundary controls. With $\nu$ and $(\hat{a}, \hat{b})$ as in Section 9.4 . we try to understand the influence of the choice of the 


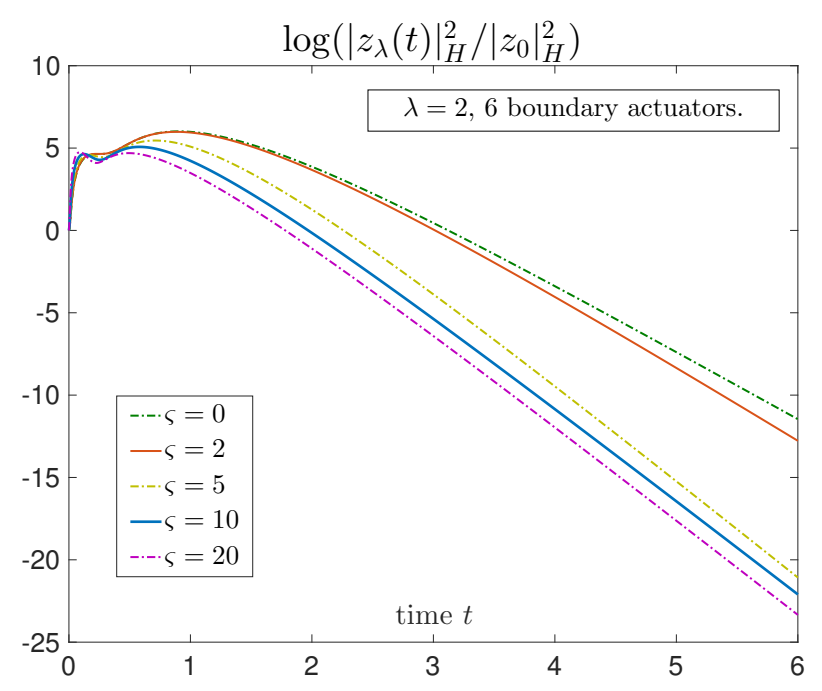

FIGURE 13. Increasing the parameter $\varsigma$.

parameter $\varsigma$ in the extended system (81). Notice that $\varsigma$ does not appear in the "original" system (81a), it is a parameter which we can choose in the dynamics of the extension variable $\kappa$ in $81 \mathrm{~b}$.

We will take 6 boundary actuators and fix $\lambda=2$. Figure 13 suggests that taking a bigger parameter $\varsigma$, we obtain a boundary feedback control providing a faster stabilization of the linear system to zero. However, we must say that taking a bigger parameter $\varsigma$, the free dynamics solution of (81b) (without the feedback control) will go faster to zero, which suggests that for bigger $\varsigma$ we may need to take smaller time step $k$ in the discretization (87) to get a good approximation of the real dynamics, which will make the simulations more expensive.

9.6. On the feedback nature of the control. Here, we remark the importance to have the control in a feedback form, because closed loop controls are known to be able to respond to small disturbances. We consider the stationary case with $\nu=\frac{1}{2}$, $\hat{a}=$ $-10+2 x_{1}+\cos x_{2}, \hat{b}=\left(-x_{1}^{2},-\sin x_{2}\right)$ as in Section 9.4 and with $\lambda=2$. However, here we consider 4 boundary actuators and set $\zeta=10$. The initial condition here is $v_{0}(x)=3+\sum_{i=1}^{4} \frac{1}{2} \widetilde{\Psi}_{i}(x)$

In Figure $14(\mathrm{a})$, we plot the results:

- for the case of the "original" close-looped system (81),

- for the case of the corresponding extended close-loop system (71) (with $(\lambda, \varsigma)$ in the role of $(\bar{\lambda}, \bar{\varsigma})$ and with $\mathbb{A}_{\lambda, \varsigma}^{\hat{a}, \hat{b}, \nu}$ as in $(78)$ in the role of $\mathbb{A}_{\bar{\lambda}, \bar{\varsigma}}^{a, b}$, and then recovering $z=y_{\lambda}+B_{\Psi} \kappa_{\lambda}$, and

- for the case where we save the $\kappa_{\lambda}$ obtained for system (71) and plug it in the system (81a) as an open-loop control.

Notice that, we do not expect that the numerical solutions obtained by solving the original closed-loop system will coincide with the recovering from the solution of the extended closed-loop system (as Figure 14(a) could suggest), but we do expect that they will be close to each other, this facts are confirmed in Figure 14(b).

For a short period of time we do not see much difference among the three procedures. For a longer period of time, the closed-loop controls are able to stabilize the corresponding systems (81) and (71) while, the open-loop control is not able to stabilize the original 


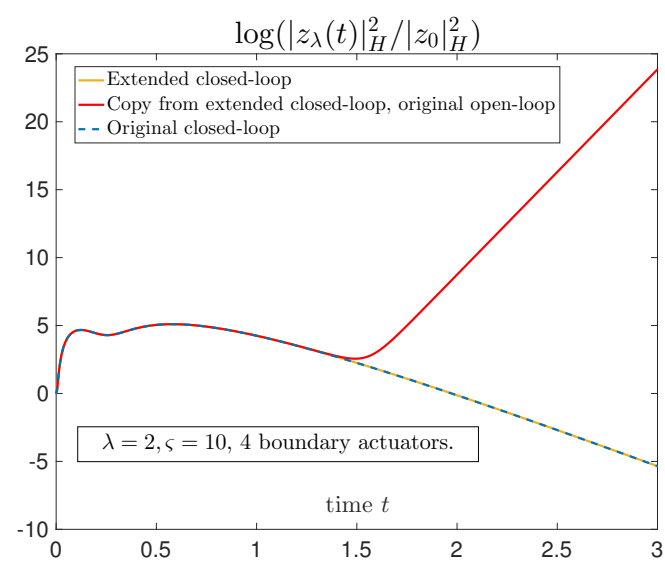

(a) Solutions from the three procedures.

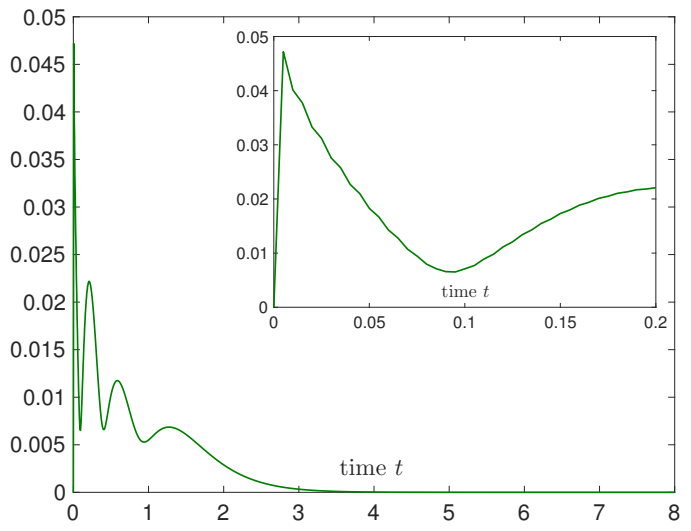

(b) The difference between solutions from extended and original closed-loop.

FiguRE 14. The "real" feedback to stabilize the system.

one (81a), even though it stabilizes the "equivalent" extended system. This is due to the fact that the discretizations errors in the original and extended systems are different, the difference between these two errors could be seen as a small disturbance to which the open-loop control cannot respond.

The conclusion is that it is important to have a control in feedback form in the original system (81). It is not enough (at the discrete level) to find the control for an "equivalent" auxiliary system, like (71), and plug it in 81a).

9.7. Switching the control off/on. With the same setting as in Section 9.6, here we perform an experiment to emphasize the importance of feedback control to stabilize the system. We set $T=6$.

In Figure 15 we plot the results for the 3 cases the control is switched on for time in $[0,6]$, for time in $[0,3] \cup[4,6]$, and for time in $[0,3]$.

Switching the control off at time instant $t=3$, we observe that the norm of the solution increases, and will (likely) not remain bounded. Notice that at time $t=3$ we have $|z(3)|_{H}^{2} \leq \mathrm{e}^{-10-3 \lambda}\left|z_{0}\right|_{H}^{2}<1.2 \times 10^{-7}\left|z_{0}\right|_{H}^{2}$ is already quite small. We also see that by switching on the control again, from time $t=4 \mathrm{on}$, then we recover the stability of the system.

Notice that at time $t=3$ and $t=4$, that is, we do not know the "analytical" expressions for $z(3)$ and $z(4)$ which come from the numerical solution. Therefore, in some sense, $z(3)$ and $z(4)$ can be seen as "random" "initial" conditions showing the instability of the system and the stabilizing property of the feedback control.

9.8. A nonlinear example. We consider the following nonlinear parabolic equation, for time $t \in[0, T]$, again in the unit ball $\Omega=\mathbb{D}=\left\{x=\left(x_{1}, x_{2}\right) \in \mathbb{R}^{2} \mid x_{1}^{2}+x_{2}^{2}<1\right\}$.

$$
\begin{aligned}
\partial_{t} y-\nu \Delta y+c_{3} y^{3}+c_{2} y^{2}+c_{1} y+\frac{1}{2} \nabla \cdot\left(y^{2}, y^{2}\right)+f_{0} & =0,\left.\quad y\right|_{\Gamma}=g, \\
y(0, x) & =y_{0}(x),
\end{aligned}
$$

where $c_{1}, c_{2}$, and $c_{3}$ are constants in $\mathbb{R}$, and $f_{0}$ is a fixed appropriate function. 


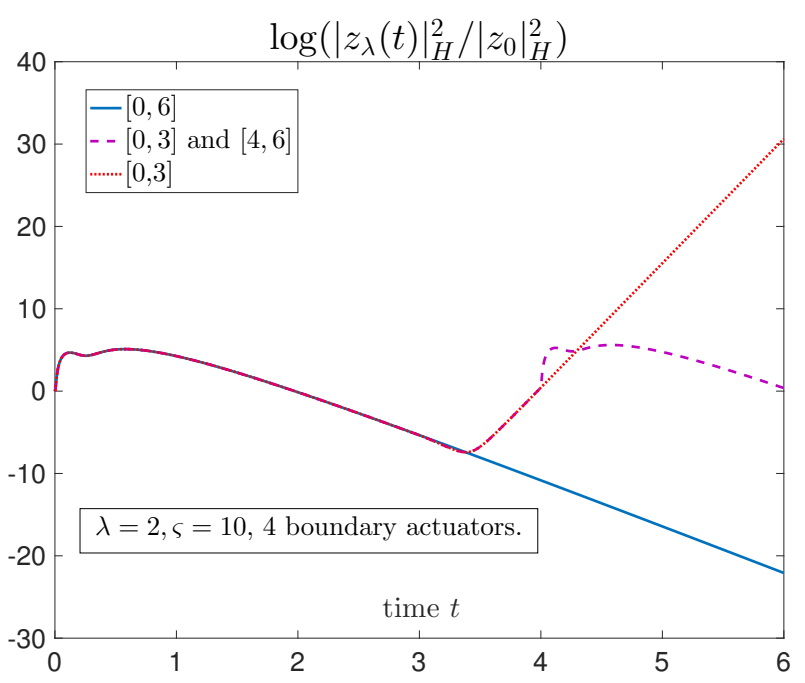

FIGURE 15. Switch on and switch off the feedback control.

Let us fix a smooth function $\hat{y}$ which we will take as our reference trajectory. Then, as external forces, we (must) take the functions

$$
\begin{aligned}
f_{0} & =f_{0}(\hat{y})=-\left(\partial_{t} \hat{y}-\nu \Delta \hat{y}+c_{3} \hat{y}^{3}+c_{2} \hat{y}^{2}+c_{1} \hat{y}+\frac{1}{2} \nabla \cdot\left(\hat{y}^{2}, \hat{y}^{2}\right)\right), \\
g & =g(\hat{y})=\left.\hat{y}\right|_{\Gamma},
\end{aligned}
$$

We will also set the parameters

$$
\nu=0.2, \quad\left(c_{1}, c_{2}, c_{3}\right)=(-2,-1,-3), \quad \lambda=1 .
$$

Remark 9.1. In order to make a smooth function $\hat{y}(t, x)$ a solution of $(96)$, we have just to set the appropriate external forces $f_{0}$ and $g$ as in (97). Thus, to test with other reference trajectories, we would just have to take the corresponding $f_{0}$ and $g$.

9.8.1. Discretization. Given a triangular mesh $\mathbf{D}=(\mathbf{p}, \mathbf{e}, \mathbf{t})$ of $\mathbb{D}$ and a time step $k>0$, we discretize system (96) as if it were the heat equation, simply looking at the nonlinearity as an external forcing, that is, writing

$$
\begin{aligned}
f_{1}(y, \nabla y) & =c_{3} y^{3}+c_{2} y^{2}+c_{1} y+\frac{1}{2} \nabla \cdot\left(y^{2}, y^{2}\right), \\
f=f(y, \nabla y) & =f_{1}(y, \nabla y)+f_{0},
\end{aligned}
$$

since $f_{0}$ and $g$ are smooth we can take at each time step $j k$, as approximations of $f_{0}(j k)$ and $g(j k)$ the corresponding evaluation vectors $\overline{f_{0}(j k)}$ and $\overline{g(j k)}$ at the points $\mathbf{p}$ of $\mathbf{D}$.

For the nonlinear terms, at each time step, we take the aproximations

$$
\left(c_{r} y^{r}\right)^{j}:=c_{r} \mathbf{M}\left(\bar{y}^{j}\right)^{r} \approx c_{r} y^{r}(j k), \quad \frac{1}{2}\left(\mathbf{G}_{x_{1}}\left(\bar{y}^{j}\right)^{2}+\mathbf{G}_{x_{2}}\left(\bar{y}^{j}\right)^{2}\right) \approx \frac{1}{2} \nabla \cdot\left(y^{2}(j k), y^{2}(j k)\right),
$$

where $\left(\bar{y}^{j}\right)^{r}$ means that we take the $r$-th power of each coordinate of $\bar{y}^{j}$. Hence, given vector solution $\bar{y}^{j}$, at time $j k$, we approximate the nonlinearity, as a function from $V$ into $V^{\prime}$, as

$$
\mathcal{N}_{1, D}\left(\bar{y}^{j}\right):=\mathbf{M}\left(c_{3}\left(\bar{y}^{j}\right)^{3}+c_{2}\left(\bar{y}^{j}\right)^{2}+c_{1} \bar{y}^{j}\right)+\frac{1}{2}\left(\mathbf{G}_{x_{1}}\left(\bar{y}^{j}\right)^{2}+\mathbf{G}_{x_{2}}\left(\bar{y}^{j}\right)^{2}\right) .
$$

We arrive to a semi-discretization analogously to 83 :

$$
\partial_{t} \mathbf{M}_{\mathrm{ii}} \bar{y}_{\mathrm{i}}=-\nu \mathbf{S}_{\mathrm{ii}} \bar{y}_{\mathrm{i}}-\nu \mathbf{S}_{\mathrm{ib}} \bar{g}-\partial_{t} \mathbf{M}_{\mathrm{ib}} \bar{g}-\left[\begin{array}{ll}
\mathbf{M}_{\mathrm{ii}} & \mathbf{M}_{\mathrm{ib}}
\end{array}\right] \bar{f}_{0}-\left(\mathcal{N}_{1, D}(\bar{y})\right)_{\mathrm{i}} .
$$


where $\left(\mathcal{N}_{1, D}(\bar{y})\right)_{\text {i }}$ stands for the coordinates of the vector $\mathcal{N}_{1, D}(\bar{y})$ corresponding to the interior points of the mesh. As before $\bar{y}=\left[\begin{array}{c}\bar{y}_{\mathrm{i}} \\ \bar{y}_{\mathrm{b}}\end{array}\right]=\left[\begin{array}{c}\bar{y}_{\mathrm{i}} \\ \bar{g}\end{array}\right]$.

Then, with Crank-Nicolson scheme, and with the notations as in (91b), we find

$$
\begin{aligned}
\mathbf{A}_{\mathrm{ii}}^{\oplus} \bar{y}_{\mathrm{i}}^{j+1}= & \mathbf{A}_{\mathrm{ii}}^{\ominus} \bar{y}_{\mathrm{i}}^{j}-\mathbf{A}_{\mathrm{ib}}^{\oplus \bar{g}^{j+1}}+\mathbf{A}_{\mathrm{ib}}^{\ominus} \bar{g}^{j}-k\left[\mathbf{M}_{\mathrm{ii}} \quad \mathbf{M}_{\mathrm{ib}}\right]\left(\bar{f}_{0}^{j+1}+\bar{f}_{0}^{j}\right) \\
& -k\left(\left(\mathcal{N}_{1, D}\left(\bar{y}^{j+1}\right)\right)_{\mathrm{i}}+\left(\mathcal{N}_{1, D}\left(\bar{y}^{j}\right)\right)_{\mathrm{i}}\right) .
\end{aligned}
$$

Knowing $y_{\mathrm{i}}^{j}$, the only unknown term in the right hand side is $\left(\mathcal{N}_{1, D}\left(\bar{y}^{j+1}\right)\right)_{\mathrm{i}}$. To approximate this term, we take again the linear extrapolation

$$
\left(\mathcal{N}_{1, D}\left(\bar{y}^{j+1}\right)\right)_{\mathrm{i}}=2\left(\mathcal{N}_{1, D}\left(\bar{y}^{j}\right)\right)_{\mathrm{i}}-\left(\mathcal{N}_{1, D}\left(\bar{y}^{j-1}\right)\right)_{\mathrm{i}}, \quad j \geq 0, \quad \text { with } \quad\left(\mathcal{N}_{1, D}\left(\bar{y}^{-1}\right)\right)_{\mathrm{i}}:=\left(\mathcal{N}_{1, D}\left(\bar{y}^{0}\right)\right)_{\mathrm{i}} .
$$

Therefore we arrive to the scheme

$$
\begin{aligned}
\mathbf{A}_{\mathrm{ii}}^{\oplus} \bar{y}_{\mathrm{i}}^{j+1}= & \mathbf{A}_{\mathrm{ii}}^{\ominus} \bar{y}_{\mathrm{i}}^{j}-\mathbf{A}_{\mathrm{ib}}^{\oplus \bar{g}^{j+1}}+\mathbf{A}_{\mathrm{ib}}^{\ominus} \bar{g}^{j}-k\left[\mathbf{M}_{\mathrm{ii}} \quad \mathbf{M}_{\mathrm{ib}}\right]\left(\bar{f}_{0}^{j+1}+\bar{f}_{0}^{j}\right) \\
& \left.-k\left(3\left(\mathcal{N}_{1, D}\left(\bar{y}^{j}\right)\right)_{\mathrm{i}}-\mathcal{N}_{1, D}\left(\bar{y}^{j-1}\right)\right)_{\mathrm{i}}\right),
\end{aligned}
$$

which we can invert to obtain $\bar{y}_{\mathrm{i}}^{j+1}$.

9.8.2. Local feedback stabilization. With the setting as in (9.8), we take $T=8$ and the reference trajectory

$$
\hat{y}(t)=\left(2 x_{1}^{3}+x_{2}^{2}\right) \sin t
$$

which solves (96), provided we take the "fixed" external forces as in (97).

We will confirm that the feedback control is able to stabilize locally system (96) to the targetted trajectory $\hat{y}$ (see Theorems 5.1 and 7.15 with exponential rate $\frac{\lambda}{2}$. That is, the solutions of the systems

$$
\begin{aligned}
\partial_{t} y-\nu \Delta y+f_{1}(y, \nabla y)+f_{0}+B_{M} B_{M}^{*} \widehat{\Pi}_{\lambda}(y-\hat{y}) & =0,\left.\quad y\right|_{\Gamma}=g, \\
y(0) & =y_{0},
\end{aligned}
$$

and

$$
\begin{aligned}
\partial_{t} y-\nu \Delta y+f_{1}(y, \nabla y)+f_{0} & =0, & \left.z\right|_{\Gamma} & =B_{\Psi}^{\Gamma} \kappa, \\
\partial_{t} \kappa+\varsigma \kappa+\mathcal{F}^{\mathrm{bo}}\left(y-\hat{y}-B_{\Psi} \kappa, \kappa\right) & =0, & (y(0), \kappa(0)) & =\left(y_{0}, \kappa_{0}\right) .
\end{aligned}
$$

go exponential to $\hat{y}$, with rate $\frac{\lambda}{2}$, provided the initial condition $y_{0}$ is close enough to $\hat{y}_{0}$. On the other hand, $y_{u}$ is the solution of systems (102) and (103) without any feedback control.

The feedback control is found to stabilize, respectively, the linearized systems (80) and (81). That is, respectively, by solving the differential Riccati equations (55) and (77), with $\hat{a}$ and $\hat{b}$ as in $(5)$.

Departing from (101), and proceeding as for the systems (90) and (91), by taking a suitable linear extrapolations at time $t=(j+1) k$, we arrive to the following discretizations of the systems (102) and (103).

In the internal case we arrive to

$$
\begin{aligned}
\mathbf{A}_{\mathrm{ii}}^{\oplus} \bar{y}_{\mathrm{i}}^{j+1}= & \mathbf{A}_{\mathrm{ii}}^{\ominus} \bar{y}_{\mathrm{i}}^{j}-\mathbf{A}_{\mathrm{ib}}^{\oplus \bar{g}^{j+1}}+\mathbf{A}_{\mathrm{ib}}^{\ominus} \bar{g}^{j}-k\left[\mathbf{M}_{\mathrm{ii}} \quad \mathbf{M}_{\mathrm{ib}}\right]\left(\bar{f}_{0}^{j+1}+\bar{f}_{0}^{j}\right) \\
& \left.-k\left(3\left(\mathcal{N}_{1, D}\left(\bar{y}^{j}\right)\right)_{\mathrm{i}}-\mathcal{N}_{1, D}\left(\bar{y}^{j-1}\right)\right)_{\mathrm{i}}\right)-k \mathbf{M}_{\mathrm{ii}}\left(3 \mathcal{F}^{j, \mathrm{in}} \bar{y}_{\mathrm{i}}^{j}-\mathcal{F}^{j-1, \mathrm{in}} \bar{y}_{\mathrm{i}}^{j-1}\right) .
\end{aligned}
$$


In the boundary case we arrive to

$$
\begin{aligned}
(2+k \varsigma) \kappa^{j+1}= & (2-k \varsigma) \kappa^{j}-k\left(3 \mathcal{F}_{\mathrm{b}}^{j, \mathrm{bo}}\left[\begin{array}{l}
\bar{y}_{\mathrm{i}}^{j} \\
\kappa^{j}
\end{array}\right]-\mathcal{F}_{\mathrm{b}}^{j-1, \mathrm{bo}}\left[\begin{array}{l}
\bar{y}_{\mathrm{i}}^{j-1} \\
\kappa^{j-1}
\end{array}\right]\right), \\
\mathbf{A}_{\mathrm{ii}}^{\oplus} \bar{y}_{\mathrm{i}}^{j+1}= & \mathbf{A}_{\mathrm{ii}}^{\ominus} \bar{y}_{\mathrm{i}}^{j}-\mathbf{A}_{\mathrm{ib}}^{\oplus}\left(\bar{g}^{j+1}+B \frac{\Gamma}{\bar{\Psi}^{j+1}}\right)+\mathbf{A}_{\mathrm{ib}}^{\ominus}\left(\bar{g}^{j}+B \frac{\Gamma}{\bar{\Psi}^{j}}\right) \\
& \left.-k\left[\begin{array}{ll}
\mathbf{M}_{\mathrm{ii}} & \mathbf{M}_{\mathrm{ib}}
\end{array}\right]\left(\bar{f}_{0}^{j+1}+\bar{f}_{0}^{j}\right)-k\left(3\left(\mathcal{N}_{1, D}\left(\bar{y}^{j}\right)\right)_{\mathrm{i}}-\mathcal{N}_{1, D}\left(\bar{y}^{j-1}\right)\right)_{\mathrm{i}}\right),
\end{aligned}
$$

where $\mathcal{N}_{1, D}\left(\bar{y}^{j}\right)$ is as in $(98)$ and the feedback rules $\mathcal{F}^{\text {in }}=\mathcal{F}_{\lambda}^{\text {in }}$ and $\mathcal{F}^{\text {bo }}=\mathcal{F}_{\lambda}^{\text {bo }}$ computed as in Section 8.1.4.

The case of internal controls. For system (102), we take the initial condition in the form $y_{0}=y_{0}(x)=\hat{y}_{0}(x)+\epsilon v_{0}(x)$, with $v_{0}$ chosen as

$$
v_{0}(x):= \begin{cases}1, & \text { if } x<\frac{-1}{3} \\ 0, & \text { otherwise }\end{cases}
$$

We take 6 piecewise constant actuators, as in Section 9.2.1, supported in the rectangular subset $\omega$ corresponding to the arrangement $(m, n)=(3,2)$.

In Figure 16(a), with some values of $\epsilon$ in $[-0.577,0.495]$, we see that the system is stable under the feedback control action. However, in Figure 16(b,c), feedback control can not stabilize anymore the system for $\epsilon \in\{-0.578,0.496\}$.

In Figure 17, we see that uncontrolled solution is not stable. Actually, we observe that even for small $\epsilon$ the solution explodes at some time in $(0,8)$. We would like to recall that such blowing-up is somehow expected. We refer to [Bal77, Section 3].

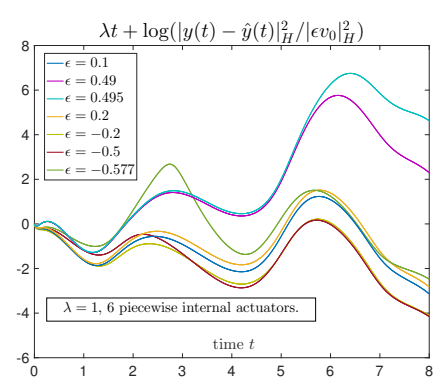

(a) With $\epsilon \in[-0.577,0.495]$.

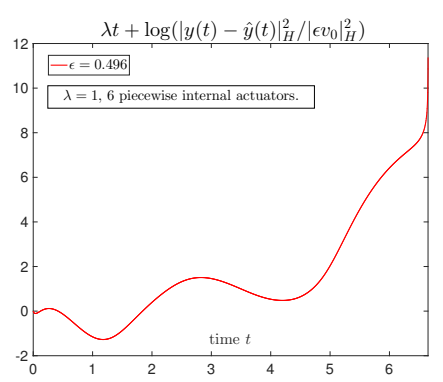

(b) With $\epsilon=0.496$.

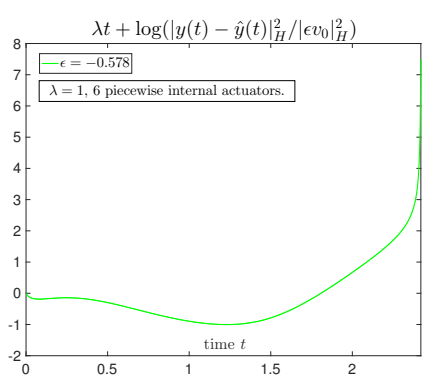

(c) With $\epsilon=-0.578$.

Figure 16. Internal feedback control. Convergence rate to $\hat{y}$ holds for small $\epsilon$.

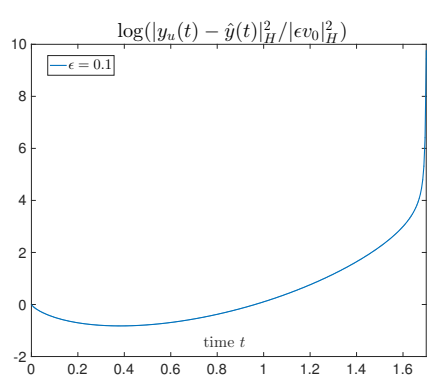

(a) With $\epsilon=0.1$.

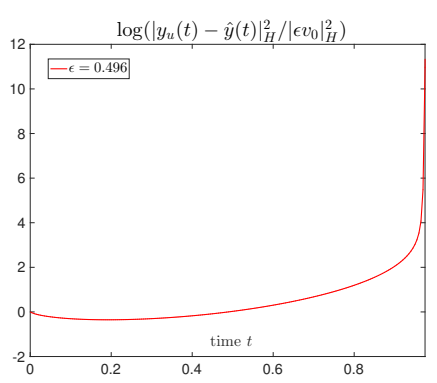

(b) With $\epsilon=0.496$.

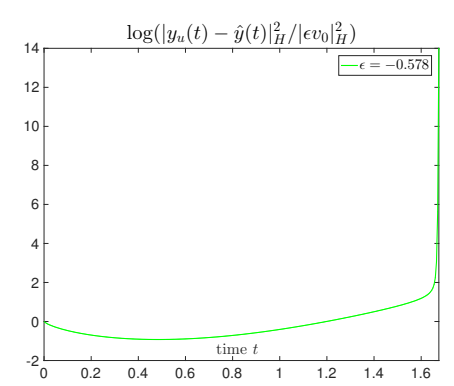

(c) With $\epsilon=-0.578$.

FIGURE 17. Uncontrolled solution. 
Notice that, Theorem 5.1 holds for $v_{0} \in V$. The above choice does not satisfy this requirement, though the numerical results show that, in this example, the feedback is still stabilizing the system locally. Next, with the same actuators, we perform another simulation with $v_{0} \in V$ being the (numerical) solution of the elliptic system

$$
-\mu \Delta v_{0}+\beta_{\mathrm{r}} v_{0}+\nabla \cdot\left(\beta_{\mathrm{c}} v_{0}\right)+h=0,\left.\quad v_{0}\right|_{\Gamma}=0,
$$

observing the analogous behaviour. Here, we choose

$$
\begin{aligned}
\mu=0.5, \quad \beta_{\mathrm{r}}\left(x_{1}, x_{2}\right) & =\sin \left(x_{1}\right)+x_{2}, \quad \beta_{\mathrm{c}}\left(x_{1}, x_{2}\right)=\left(2 x_{1} x_{2},-2 \sin \left(x_{2}\right)\right), \\
\text { and } \quad h\left(x_{1}, x_{2}\right) & =\cos ^{2}(3 y)+\sin (x)+2 .
\end{aligned}
$$

In Figure 18(a), we plot the solution $v_{0}$ of the equation above. Again, in Figure 18 , we observe that for small $\epsilon \in[-0.14,0.1511]$ the feedback control is able to stabilize the system. In Figure 19, the uncontrolled system is still unstable and exploding.

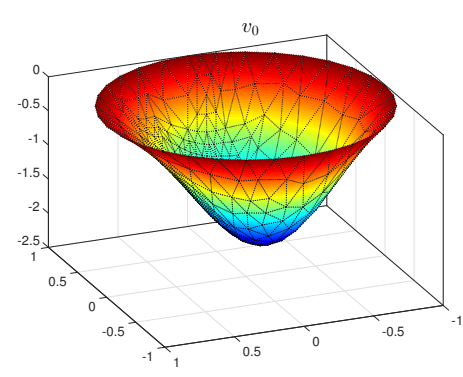

(a) The function $v_{0}$.

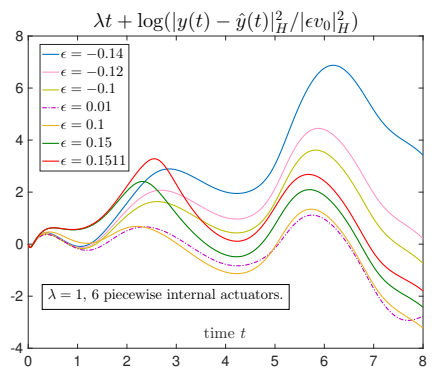

(b) With $\epsilon \in[-0.14,0.1511]$.

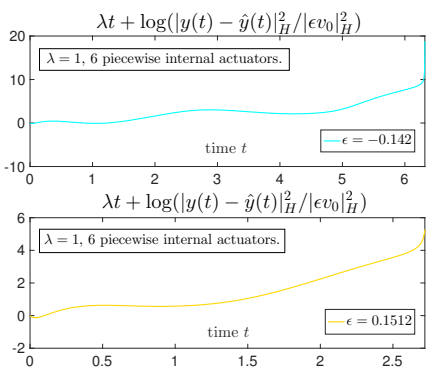

(c) With $\epsilon \in\{-0.142,0.1512\}$.

FiguRE 18. Internal feedback control. Convergence rate to $\hat{y}$ holds for small $\epsilon$.

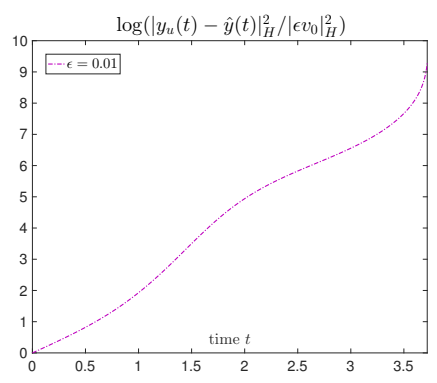

(a) With $\epsilon=0.01$.

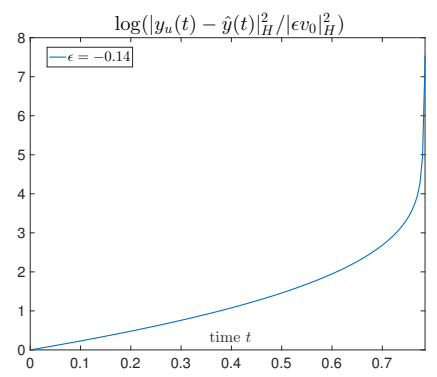

(b) With $\epsilon=-0.14$.

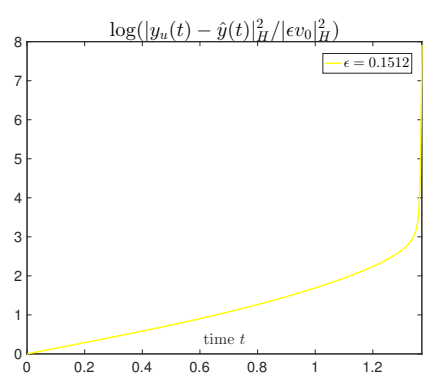

(c) With $\epsilon=0.1512$.

Figure 19. Uncontrolled solution.

The case of boundary controls. Next, we use 6 boundary actuators as in Section 9.2.2, see (93). Here in order to guarantee the compability condition (75), we take $y_{0}=\hat{y}_{0}+\epsilon v_{0}$ where $v_{0}$ is obtained by solving an elliptic equation

$$
-\mu \Delta v_{0}+\beta_{\mathrm{r}} v_{0}+\nabla \cdot\left(\beta_{\mathrm{c}} v_{0}\right)+h=0,\left.\quad v_{0}\right|_{\Gamma}=\sum_{i=1}^{M} \varrho_{i} \Psi_{i},
$$

with $\mu, \beta_{\mathrm{r}}, \beta_{\mathrm{c}}$, and $h$ as in 104 and with $\varrho=\left[\begin{array}{llllll}1 & 1 & 0 & 0.5 & 0 & 0\end{array}\right]^{\top}$. In Figure 20(a), we plot the function $v_{0}$ that we get by solving the system above.

Again, solving our system for different values of $\epsilon$, in Figures 20(b) we observe that under the boundary feedback control, is stable for small $\epsilon$. The feedback fails to stabilize 
the system for bigger $\epsilon$, as we see in Figure 20(c). In this case we take $\kappa^{0}=\kappa(0)=\epsilon \varrho$ in (91a).

In Figure 21 we see that the uncontrolled solution is not stable, and it even explodes for small $\epsilon$.

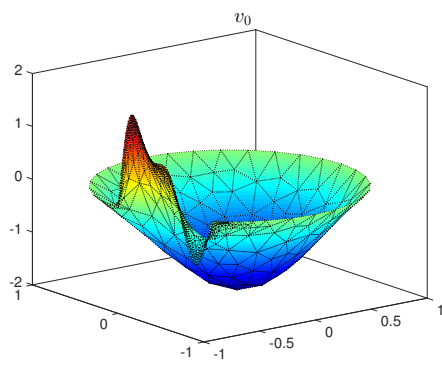

(a) The function $v_{0}$.

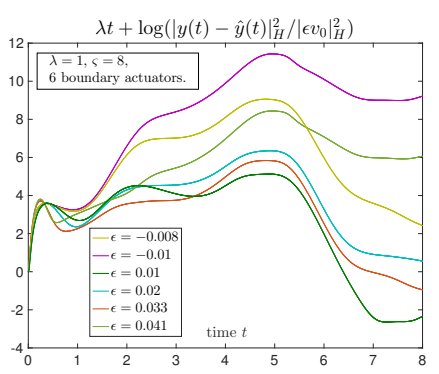

(b) With $\epsilon \in[-0.008,0.041]$.

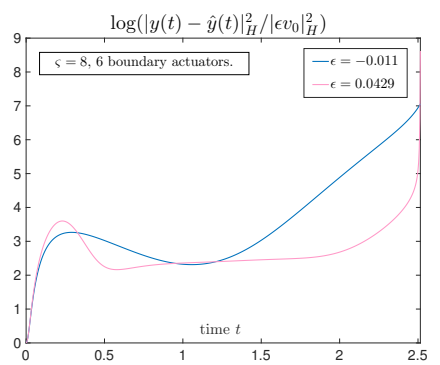

(c) With $\epsilon \in\{-0.011,0.0429\}$.

Figure 20. Boundary feedback control. Convergence rate to $\hat{y}$ holds for small $\epsilon$.

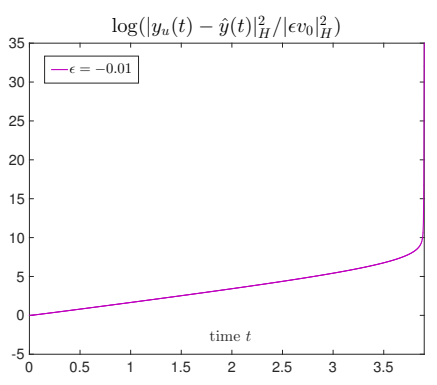

(a) With $\epsilon=-0.01$.

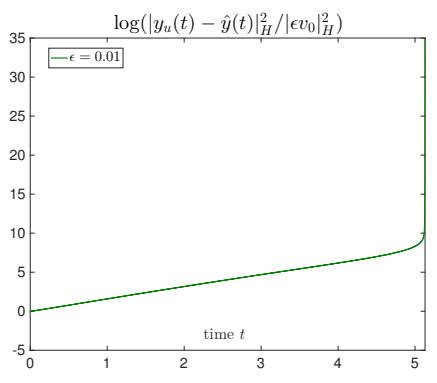

(b) With $\epsilon=0.01$.

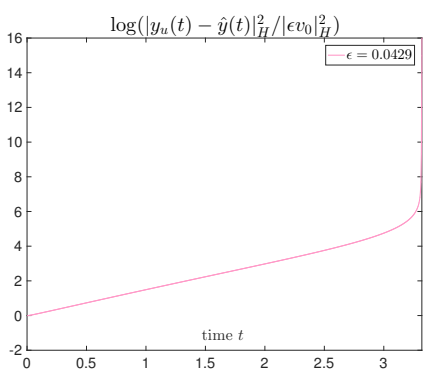

(c) With $\epsilon=0.0429$.

FigURE 21. Uncontrolled solution.

9.9. The discretization error. Here we take the time interval $[0,5]$ and consider the function

$$
\hat{y}\left(x_{1}, x_{2}\right)=\left(t^{2}-2 t\right) x_{1}^{3} \sin ^{2}\left(x_{2}\right) .
$$

Of course we expect the discrete solution vector $\bar{y}$ to get closer to $\overline{\hat{y}}$ as the triangular mesh in $\mathbb{D}$ gets finer and the timestep gets smaller. Here we show, just through a simulation that this is the case for the discretization we propose.

We check the error that we obtain as the mesh pair $(\mathbf{D}, k)$ is refined. We start with a pair $\left(\mathbf{D}_{1}, k_{1}\right)$ where $\mathbf{D}_{1}$ is a triangular mesh of the cylinder $\mathbb{D}$, where each edge of each triangle in the mesh has a length bounded above by $h_{1}=0.6$, and $k_{1}=0.01$ is the time step. More precisely $\mathbf{D}_{1}$ was generated by the MATLAB function initmesh with the input $\operatorname{Hmax}=0.6$.

Recursively, we construct the finer pair $\left(\mathbf{D}_{r+1}, k_{r+1}\right)$ by refining regularly the triangulation $\mathbf{D}_{r}$ (by connecting the middle points of the edges of each triangle), and by dividing the time-step by $2, k_{r+1}=\frac{k_{r}}{2}$. Hence, we want the solution $y[r]$ obtained with $\left(\mathbf{D}_{r}, k_{r}\right)$ to converge to $\hat{y}$ as $r$ increases.

We will also compare our approach with a Newton and a Heun based approach. The methods differ in the way we solve 99 . 
Remark 9.2. We follow [Ros08, Section 7.2.2] and [McD07, Section 4.1.2] for the terminology "Heun approach" or "Heun method". However, in different references the terminology may vary, for example we find "Modified Euler method" in [BF10, Section 5.4], or "Explicit Trapezoidal method" in [AP98, Section 4.1].

The Heun based approach. Here instead of the extrapolation 100 it is used an explicit Euler step, see [McD07], to find a preliminary guess $y_{\mathrm{i}}^{*}$ for $y_{\mathrm{i}}^{j+1}$ as

$$
\begin{aligned}
\mathbf{M}_{\mathrm{ii}} \bar{y}_{\mathrm{i}}^{*}= & \left(\mathbf{M}_{\mathrm{ii}}-k \nu \mathbf{S}_{\mathrm{ii}}\right) \bar{y}_{\mathrm{i}}^{j}+\left(\mathbf{M}_{\mathrm{ib}}-k \nu \mathbf{S}_{\mathrm{ib}}\right) \bar{g}^{j} \\
& -\mathbf{M}_{\mathrm{ib}} \bar{g}^{j+1}-k\left[\begin{array}{ll}
\mathbf{M}_{\mathrm{ii}} & \mathbf{M}_{\mathrm{ib}}
\end{array}\right] \bar{f}_{0}^{j}-k\left(\mathcal{N}_{1, D}\left(\bar{y}^{j}\right)\right)
\end{aligned}
$$

By defining $\bar{y}_{\mathbf{G}}=\left[\begin{array}{l}\bar{y}_{i}^{*} \\ \bar{g}^{j}\end{array}\right]$, we arrive to the scheme

$$
\begin{aligned}
\mathbf{A}_{\mathrm{ii}}^{\oplus} \bar{y}_{\mathrm{i}}^{j+1}= & \mathbf{A}_{\mathrm{ii}}^{\ominus} \bar{y}_{\mathrm{i}}^{j}-\mathbf{A}_{\mathrm{ib}}^{\oplus} \bar{g}^{j+1}+\mathbf{A}_{\mathrm{ib}}^{\ominus} \bar{g}^{j}-k\left[\mathbf{M}_{\mathrm{ii}} \quad \mathbf{M}_{\mathrm{ib}}\right]\left(\bar{f}_{0}^{j+1}+\bar{f}_{0}^{j}\right) \\
& -k\left(\left(\mathcal{N}_{1, D}\left(\bar{y}_{\mathbf{G}}\right)\right)_{\mathrm{i}}+\left(\mathcal{N}_{1, D}\left(\bar{y}^{j}\right)\right)_{\mathrm{i}}\right) .
\end{aligned}
$$

The Newton based approach. We solve (99), by a fixed point iterative procedure, see [BF10, Section 10.2]. We write (99) in the form

$$
F\left(\bar{y}_{\mathrm{i}}^{j+1}\right)=0,
$$

with

$$
\begin{aligned}
F(w) & :=-\mathbf{A}_{\mathrm{ii}}^{\oplus} w-k\left(\mathcal{N}_{1, D}\left(\left[\begin{array}{c}
w \\
\bar{g}^{j+1}
\end{array}\right]\right)\right)_{\mathrm{i}}+H^{j}, \\
H^{j} & :=\mathbf{A}_{\mathrm{ii}}^{\ominus} \bar{y}_{\mathrm{i}}^{j}-\mathbf{A}_{\mathrm{ib}}^{\oplus} \bar{g}^{j+1}+\mathbf{A}_{\mathrm{ib}}^{\ominus} \bar{g}^{j}-k\left[\mathbf{M}_{\mathrm{ii}} \quad \mathbf{M}_{\mathrm{ib}}\right]\left(\bar{f}_{0}^{j+1}+\bar{f}_{0}^{j}\right)-k \mathcal{N}_{1, D}\left(\bar{y}^{j}\right)_{\mathrm{i}} .
\end{aligned}
$$

Next, we take the derivative of $F$ at a given vector $w^{0}$ :

$$
\begin{aligned}
\mathrm{d} F_{w^{0}} & :=-\mathbf{A}_{\mathrm{ii}}^{\oplus}-k\left(\mathbf{M}\left(3 c_{3}\left[\begin{array}{c}
w^{0} \\
\bar{g}^{j+1}
\end{array}\right]^{2}+2 c_{2}\left[\begin{array}{c}
w^{0} \\
\bar{g}^{j+1}
\end{array}\right]+c_{1}\right)+\mathbf{G}_{x_{1}}\left[\begin{array}{c}
w^{0} \\
\bar{g}^{j+1}
\end{array}\right]+\mathbf{G}_{x_{2}}\left[\begin{array}{c}
w^{0} \\
\bar{g}^{j+1}
\end{array}\right]\right)_{\mathrm{i}} \\
& =A_{w_{0}}^{N}+B\left(\bar{g}^{j+1}\right),
\end{aligned}
$$

with

$$
\begin{aligned}
A_{w_{0}}^{N} & :=-\mathbf{A}_{\mathrm{ii}}^{\oplus}-k\left(\mathbf{M}_{\mathrm{ii}}\left(3 c_{3}\left(w^{0}\right)^{2}+2 c_{2} w^{0}+c_{1}\right)+\mathbf{G}_{x_{1}, \mathrm{ii}} w^{0}+\mathbf{G}_{x_{2}, \mathrm{ii}} w^{0}\right), \\
B\left(\bar{g}^{j+1}\right) & :=-k\left(\mathbf{M}_{\mathrm{ib}}\left(3 c_{3}\left(\bar{g}^{j+1}\right)^{2}+2 c_{2} \bar{g}^{j+1}+c_{1}\right)+\mathbf{G}_{x_{1}, \mathrm{ib}} \bar{g}^{j+1}+\mathbf{G}_{x_{2}, \mathrm{ib}} \bar{g}^{j+1}\right),
\end{aligned}
$$

where the powers of vectors are understood to be taken coordinate-wise.

Now we find $\bar{y}_{\mathrm{i}}^{j+1}$ iteratively, see [GT74] or [BF10, Section 10.2], as (or close to) the limit of

$$
w^{n+1}=w^{n}-\left[F^{\prime}\left(w_{n}\right)\right]^{-1} F\left(w_{n}\right) .
$$

By a continuity argument, if the time-step is small enough, we can expect the method to converge when we take the starting vector $w^{0}=\bar{y}_{\mathrm{i}}^{j}$. Another option, see [McD07, page 88], is to take $w^{0}=\bar{y}_{\mathrm{i}}^{*}$ as in (105), which may allow us to reduce the number of iterations. We used the latter in the simulations presented here. The stopping criteria which we used was
i. $\left|w^{n+1}-w^{n}\right|_{L^{\infty}}<$ tol.
ii. $\frac{\left|w^{n+1}-w^{n}\right|_{L^{\infty}}}{\left|w^{n}\right|_{L}}<$ tol.
iii. $F\left(w^{n+1}\right)<$ tol. 
with tolerance tol $=$ eps, where eps $\approx 10^{-16}$ is the MATLAB's epsilon (i.e., "zero") for double precision. Figure 22(a), shows the $L_{2}$-norm, $|y[r]-\hat{y}|_{L^{2}\left((0,5), L^{2}(\Omega, \mathbb{R})\right)}$ in the cylinder, of the discretization error with $r \in\{1,2,3,4,5\}$. The Bochner norm $|v|_{L^{2}\left((0,5), L^{2}(\Omega, \mathbb{R})\right)}$ is to be understood as the discrete approximation

$$
\left(\underline{|v|_{H}}\right)^{\top} \mathbf{M}_{\mathrm{t}} \underline{|v|_{H}} \approx \int_{0}^{5}|v(t)|_{H}|v(t)|_{H} \mathrm{~d} t=|v|_{L^{2}\left((0,5), L^{2}(\Omega, \mathbb{R})\right)}^{2}
$$

where $\underline{|v|_{H}}$ is a column vector with $N_{t}+1$ components where each component is the discrete approximation of the norm $|v(j k)|_{H}$, that is, $\left(\underline{|v|_{H}}\right)_{j}:=\sqrt{\left(\bar{v}^{j}\right)^{\top} \mathbf{M} \bar{v}^{j}}$; and $\mathbf{M}_{\mathrm{t}}$ is the mass matrix associated with the regular time mesh as in (88), with $T=5$. That is, (cf. [KR15b, Section 5.1])

$$
\mathbf{M}_{\mathrm{t}}:=\frac{k}{6}\left[\begin{array}{cccccc}
2 & 1 & 0 & 0 & \ldots & 0 \\
1 & 4 & 1 & 0 & \ldots & 0 \\
0 & 1 & 4 & 1 & \ddots & \vdots \\
\vdots & \ddots & \ddots & \ddots & \ddots & 0 \\
0 & \ldots & 0 & 1 & 4 & 1 \\
0 & \ldots & 0 & 0 & 1 & 2
\end{array}\right]
$$

In the Figure 22(b) we can see that the rate of convergence approaches 4 (i.e., second order convergence) for all the three approaches.

It is clear that the cheapest method is the one which we propose, and that the Newton method is the more expensive one. Of course the Newton based approach is expected to be the most accurate. However, we observe in the Figure 22 that, for this example, the results of these two approaches do seem to match each other.

To find a difference between these two approaches, we have to take a bigger time step. We also take the bigger time interval $[0,6]$ and $h=0.1$. In Figure 23(a) and 23(b), we can see that with only 60 time nodes, the Newton approach gives already almost the same result as with 240 time nodes. While, we can clearly see that there is an error associated with the time step for the approach that we propose using a linear extrapolation. Notice, however that the approach we use can be less expensive for 240 time nodes than the Newton approach with 60 time nodes, depending on the number of Newton iterations at each time step, which is expected to increase with the time step (in our experiment with 60 time nodes the average number of iterations was 5.233).

Another advantage of our method is that we can invert the system (101) iteratively, as we have done in our simulations by using the conjugate gradient method. With the Newton approach the associated linearization matrices, at a given time $t=j k$, may be not symmetric and so we may need to use a direct solver, for this in our simulations we have used the backslash "Y" solver from MATLAB.

The main disadvantage of the Heun method is the fact that it uses an extrapolation based on an explicit Euler guess for the solution $y^{j+1}$ at time $t=(j+1) k$, which may lead to some oscillations/fluctuations in time if the time step is not small enough. With 60 time nodes, we observed that Heun approach fails, that is, the numerical solution has exploded. As we increase the number of time nodes to 240, in Figure 23(b) we can see that the solution obtained by Heun approach is still the worst one. In Figure 23(c), with a large enough number of time nodes, more precisely 600 , we cannot see a remarkable difference among the three approaches. 


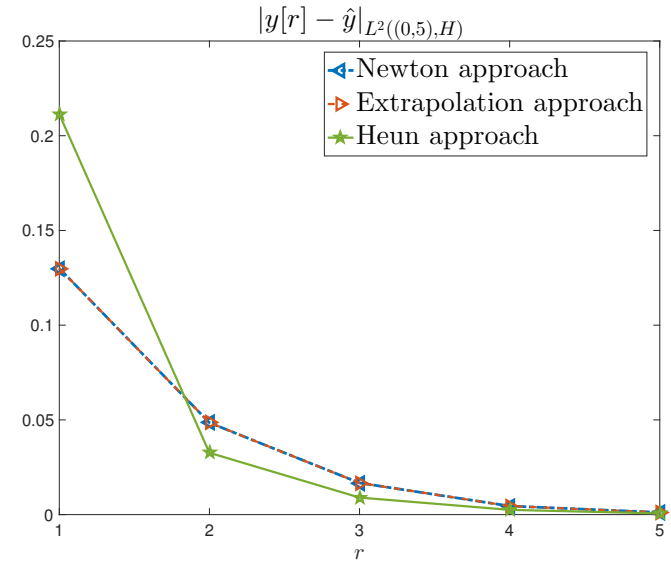

(a) $L_{2}$-norm of the error.

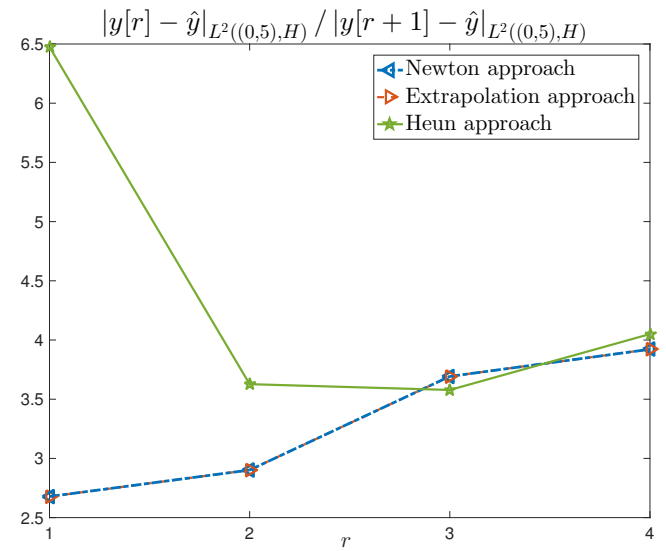

(b) Ratio between two consecutive errors.

FIGURE 22. The discretization error for the mesh pairs $\left(\mathbf{D}_{r}, k_{r}\right)$.

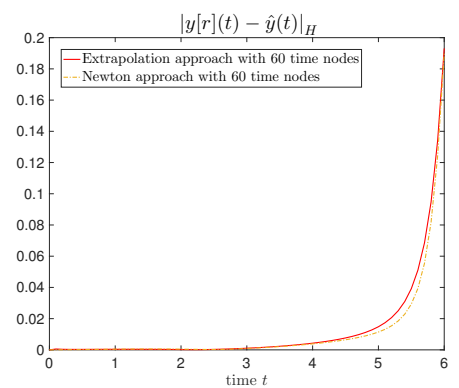

(a) With 60 time nodes

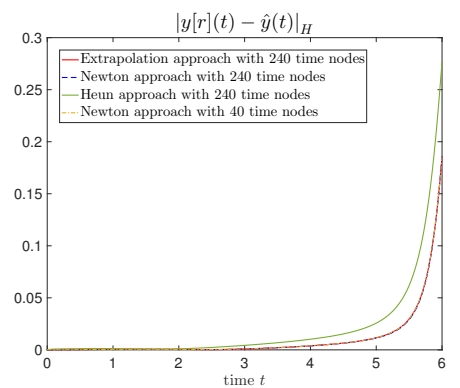

(b) With 240 time nodes.

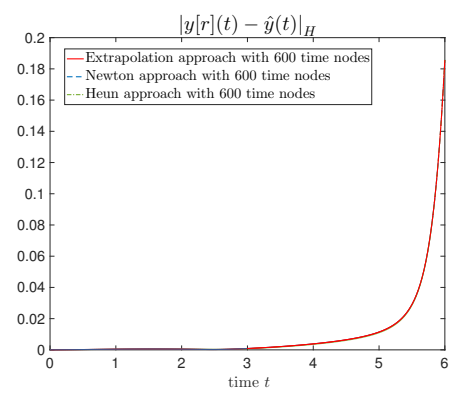

(c) With 600 time nodes.

FIGURE 23. Comparing the three approaches for different time steps.

\section{REFERENCES}

[AKBGBdT11] F. Ammar-Khodja, A. Benabdallah, M. González-Burgos, and L. de Teresa. Recent results on the controllability of linear coupled parabolic problems: a survey. Math. Control Relat. Fields, 1(3):267-306, 2011. doi:10.3934/mcrf.2011.1.267.

[AP98] U. M. Ascher and L. R. Petzold. Computer Methods for Ordinary Differential Equations and Differential-Algebraic Equations. SIAM, Philadelphia, PA, USA, 1st edition, 1998. URL: http://bookstore.siam.org/ot61/

[Bad09] M. Badra. Feedback stabilization of the 2-D and 3-D Navier-Stokes equations based on an extended system. ESAIM Control Optim. Calc. Var., 15(4):934-968, 2009. doi: $10.1051 / \mathrm{cocv}: 2008059$.

[Bal77] J. M. Ball. Remarks on blow-up and nonexistence theorems for nonlinear evolution equations. Q. J. Math., 28(4):473-486, 1977. doi:10.1093/qmath/28.4.473.

[Ben] P. Benner. Morlab package software. URL: http://www-user.tu-chemnitz.de/ ubenner/software.php

[Ben06] P. Benner. A MATLAB repository for model reduction based on spectral projection. In Proceedings of the 2006 IEEE Conference on Computer Aided Control Systems Design, pages 19-24, October 4-6 2006. doi:10.1109/CACSD-CCA-ISIC.2006.4776618.

[BF10] R. L. Burden and J. D. Faires. Numerical Analysis. Cengage Learning, 9th edition, 2010. URL: http://edu.cengage.co.uk/catalogue/product.aspx?isbn=1305253663.

[Bir13] G. D. Birkhoff. Note on the Gamma function. Bull. Amer. Math. Soc., 20(1):1-10, 1913. URL: http://projecteuclid.org/euclid.bams/1183422450.

[BKR15] T. Breiten, K. Kunisch, and S. S. Rodrigues. Feedback stabilization to non-stationary solutions of a class of reaction diffusion equations of FitzHugh-Nagumo type. 
RICAM-Report No. 2015-41 (submitted), 2015. URL: http://www.ricam. oeaw.ac .at/ publications/reports/.

[Boc33] S. Bochner. Integration von Funktionen, deren Werte die Elemente eines Vektorraumes sind. Fund. Math., 20(1):262-276, 1933. URL: https://eudml.org/doc/212635.

[BRS11] V. Barbu, S. S. Rodrigues, and A. Shirikyan. Internal exponential stabilization to a nonstationary solution for 3D Navier-Stokes equations. SIAM J. Control Optim., 49(4):1454-1478, 2011. doi:10.1137/100785739.

[BRT15] J.-M. Buchot, J.-P. Raymond, and J. Tiago. Coupling estimation and control for a two dimensional Burgers type equation. ESAIM Control Optim. Calc. Var., 21(2):535-560, 2015. doi:10.1051/cocv/2014037

[Che05] Z. Chen. Finite Element Methods and Their Applications. Springer, Berlin Heidelberg, 2005. doi:10.1007/3-540-28078-2.

[Cor07] J.-M. Coron. Control and Nonlinearity, volume 136 of Mathematical Surveys and Monographs. American Mathematical Society, Providence, RI, 2007. URL: http://www.ams. org/bookstore-getitem/item=SURV-136-S.

[DD12] F. Demengel and G. Demengel. Functional Spaces for the Theory of Elliptic Partial Differential Equations. Universitext. Springer, 2012. doi:10.1007/978-1-4471-2807-6.

[DFCGBZ02] A. Doubova, E. Fernández-Cara, M. González-Burgos, and E. Zuazua. On the controllability of parabolic systems with a nonlinear term involving the state and the gradient. SIAM J. Control Optim., 41(3):798-819, 2002. doi:10.1137/S0363012901386465.

[DZZ08] T. Duyckaerts, X. Zhang, and E. Zuazua. On the optimality of the observability inequalities for parabolic and hyperbolic systems with potentials. Ann. Inst. H. Poincaré Anal. Non Linéaire, 25(1):1-41, 2008. doi:10.1016/j.anihpc.2006.07.005.

[Fat99] H. O. Fattorini. Infinite Dimensional Optimization and Control Theory. Encyclopedia of Mathematics and its Applications. Cambridge University Press, 1999. Printing 2010. URL: http://www. cambridge.org/

[Fat05] H.O. Fattorini. Infinite Dimensional Linear Control Systems: The Time Optimal and Norm Optimal Problems. North-Holland Mathematics Studies. Elsevier Science, 2005. URL: https://books.google.at/books?id=xXNh18nglqcC

[FGP83] G. J. Fix, M. D. Gunzburger, and J. S. Peterson. On finite element approximations of problems having inhomogeneous essential boundary conditions. Comput. Math. Appl., 9(5):687-700, 1983. doi:10.1016/0898-1221(83)90126-8.

[Fis37] R. A. Fisher. The wave of advance of advantageous genes. Ann. Human Genetics, 7(4):355-369, 1937. doi:10.1111/j.1469-1809.1937.tb02153.x.

[Fol01] G. B. Folland. How to integrate a polynomial over a sphere. Amer. Math. Monthly, 108(5):446-448, 2001. URL: http://www.jstor.org/stable/2695802.

[GT74] W. B. Gragg and R. A. Tapia. Optimal error bounds for the Newton-Kantorovich theorem. SIAM J. Numer. Anal., 11(1):10-13, 1974. doi:10.1137/0711002.

[HPP01] D. Hinrichsen, E. Plischke, and A. J. Pritchard. Liapunov and Riccati equations for practical stability. In Proceedings of the 2001 European Control Conference (ECC), Porto, Portugal, pages 2883-2888, 9 2001. URL: http://ieeexplore.ieee.org/xpl/ mostRecentIssue.$j$ jsp?punumber $=7075845$.

[JK07] V. John and P. Knobloch. On spurious oscillations at layers diminishing (SOLD) methods for convection-diffusion equations: Part I - a review. Comput. Methods Appl. Mech. Engrg., 196(17-20):2197-2215, 2007. doi:10.1016/j.cma.2006.11.013.

[JK08] V. John and P. Knobloch. On spurious oscillations at layers diminishing (SOLD) methods for convection-diffusion equations: Part II - analysis for $P_{1}$ and $Q_{1}$ finite elements. Comput. Methods Appl. Mech. Engrg., 197(21-24):1997-2014, 2008. doi:10.1016/j. cma.2007.12.019

[JS08] V. John and E. Schmeyer. Finite element methods for time-dependent convectiondiffusion-reaction equations with small diffusion. Comput. Methods Appl. Mech. Engrg., 198(3-4):475-494, 2008. doi:10.1016/j.cma.2008.08.016.

[KR15a] A. Kröner and S. S. Rodrigues. Internal exponential stabilization to a nonstationary solution for 1D Burgers equations with piecewise constant controls. In Proceedings of the 2015 European Control Conference (ECC), Linz, Austria, pages 2676-2681, July 2015. doi:10.1109/ECC.2015.7330942 
[KR15b] A. Kröner and S. S. Rodrigues. Remarks on the internal exponential stabilization to a nonstationary solution for 1D Burgers equations. SIAM J. Control Optim., 53(2):10201055, 2015. doi:10.1137/140958979.

[Lio69] J.-L. Lions. Quelques Méthodes de Résolution des Problèmes aux Limites Non Linéaires. Dunod et Gauthier-Villars, Paris, 1969.

[LM72a] J.-L. Lions and E. Magenes. Non-Homogeneous Boundary Value Problems and Applications, volume I of Die Grundlehren Math. Wiss. Einzeldarstellungen. Springer-Verlag, 1972. doi:10.1007/978-3-642-65161-8

[LM72b] J.-L. Lions and E. Magenes. Non-Homogeneous Boundary Value Problems and Applications, volume II of Die Grundlehren Math. Wiss. Einzeldarstellungen. Springer-Verlag, 1972. doi:10.1007/978-3-642-65217-2.

[LY83] P. Li and S.-T. Yau. On the Schrödinger equation and the eigenvalue problem. Commun. Math. Phys., 88(3):309-318, 1983. doi:10.1007/BF01213210.

[McD07] J. M. McDonough. Lectures in basic computational numerical analysis. 2007. URL: http: //www.engr.uky.edu/ acfd/lecturenotes1.html.

[Pet38] B. J. Pettis. On integration in vector spaces. Trans. Amer. Math. Soc., 44:277-304, 1938. doi:10.1090/S0002-9947-1938-1501970-8.

[PW60] L. E. Payne and H. F. Weinberger. An optimal Poincaré inequality for convex domains. Arch. Ration. Mech. Anal., 5(1):286-292, 1960. doi:10.1007/BF00252910.

[Rod14] S. S. Rodrigues. Local exact boundary controllability of 3D Navier-Stokes equations. Nonlinear Anal., 95:175-190, 2014. doi:10.1016/j.na.2013.09.003.

[Rod15a] S. S. Rodrigues. Boundary observability inequalities for the 3D Oseen-Stokes system and applications. ESAIM Control Optim. Calc. Var., 21(3):723-756, 2015. doi:10.1051/ cocv/2014045.

[Rod15b] S. S. Rodrigues. Feedback boundary stabilization to trajectories for 3D Navier-Stokes equations. arXiv:1508.00829 [math.OC] (submitted), 2015. URL: http://arxiv.org/ abs/1508.00829.

[Ros08] S. Rosłoniec. Fundamental Numerical Methods for Electrical Engineering. Number 18 in Lecture Notes Electrical Engineering. Springer, 2008. doi:10.1007/ 978-3-540-79519-3.

[Shi11] A. Shirikyan. Control and mixing for 2D Navier-Stokes equations with space-time localised noise. arXiv:1110.0596 [math.AP], 2011. URL: http://arxiv.org/abs/1110. 0596 .

[Tem95] R. Temam. Navier-Stokes Equations and Nonlinear Functional Analysis. Number 66 in CBMS-NSF Regional Conf. Ser. Appl. Math. SIAM, Philadelphia, 2nd edition, 1995. doi:10.1137/1.9781611970050.

[Tem97] R. Temam. Infinite-Dimensional Dynamical Systems in Mechanics and Physics. Number 68 in Appl. Math. Sci. Springer, 2nd edition, 1997. doi:10.1007/ 978-1-4612-0645-3.

[Tem01] R. Temam. Navier-Stokes Equations: Theory and Numerical Analysis. AMS Chelsea Publishing, Providence, RI, reprint of the 1984 edition, 2001. URL: http://www.ams. org/bookstore-getitem/item=CHEL-343-H.

[VP09] V. Volpert and S. Petrovskii. Reaction-diffusion waves in biology. Phys. Life Rev., 6(4):267-310, 2009. doi:10.1016/j.plrev.2009.10.002.

Johann Radon Institute for Computational and Applied Mathematics, ÖAW, Altenbergerstraße 69, A-4040 Linz.

E-MAILS: duy.phan-duc@oeaw.ac.at, sergio.rodrigues@oeaw.ac . at 